\title{
QUANTUM UNIQUE ERGODICITY FOR HALF-INTEGRAL WEIGHT AUTOMORPHIC FORMS
}

\author{
STEPHEN LESTER AND MAKSYM RADZIWIŁE
}

\begin{abstract}
We investigate the analogue of the Quantum Unique Ergodicity (QUE) conjecture for half-integral weight automorphic forms. Assuming the Generalized Riemann Hypothesis (GRH) we establish QUE for both half-integral weight holomorphic Hecke cusp forms for $\Gamma_{0}(4)$ lying in Kohnen's plus subspace and for halfintegral weight Hecke Maaß cusp forms for $\Gamma_{0}(4)$ lying in Kohnen's plus subspace. By combining the former result along with an argument of Rudnick, it follows that under GRH the zeros of these holomorphic Hecke cusp equidistribute with respect to hyperbolic measure on $\Gamma_{0}(4) \backslash \mathbb{H}$ as the weight tends to infinity.
\end{abstract}

\section{Contents}

1. Introduction

1.1. Quantum Unique Ergodicity

1.2. Half-integral weight automorphic forms 3

1.3. Holomorphic half-integral weight forms 4

1.4. Half-integral weight Maaß forms 6

1.5. Comments on the proof and further work 8

2. Overview of the argument 8

2.1. Reduction to sums of Fourier coefficients 8

2.2. Estimating the sums of Fourier coefficients 10

3. Estimates for character sums 12

4. A moment calculation 16

5. Lemmas on $L$-functions 21

5.1. Heuristics 21

5.2. Rigorous results under GRH 22

6. Averages of coefficients of half-integral weight Maaß forms 32

7. Extending the length of summation 36

7.1. Proof of Lemma 7.1 Step 1: A first evaluation of (7.4). 39

7.2. Proof of Lemma 7.1. Step 2: A second evaluation of (7.4) 40

8. The summation formula and proof of QUE 42

Date: June 15, 2016.

2010 Mathematics Subject Classification. 11F37, 11F30 (primary) 11M41 (secondary). 
8.1. Estimates for Whittaker functions. 42

8.2. The summation formula 42

8.3. Proof of Quantum Unique Ergodicity 46

9. Equidistribution of mass and zeros of half-integral weight holomorphic forms

9.1. Preliminaries

48

9.2. Proof of Theorem 11 49

9.3. Equidistribution of zeros: Proof of Corollary 1.1 53

10. Appendix: Proof of Proposition 6.1

11. Appendix: The functional equation 56

12. Acknowledgments 59

References 59

\section{INTRODUCTION}

1.1. Quantum Unique Ergodicity. Let $(M, g)$ be a compact, smooth Riemannian manifold without boundary and denote by $-\Delta_{g}$ the Laplace-Beltrami operator for $M$. Also, let $\operatorname{vol}_{g}$ denote the normalized Riemannian volume form for $M$. Given an orthonormal basis $\left\{\phi_{\ell}\right\}_{\ell}$ of $L^{2}\left(M, \mathrm{dvol}_{g}\right)$-normalized eigenfunctions of $\Delta_{g}$ with eigenvalues $\lambda_{\ell}$ the Quantum Ergodicity Theorem of Shnirel'man [45], Colin de Verdiére [4] and Zelditch [54] implies that if the geodesic flow is ergodic on the unit cotangent bundle $S^{*} M$ with respect to the normalized Liouville measure then there exists a density one subsequence of eigenfunctions $\left\{\phi_{\ell_{j}}\right\}_{j}$ along which the measures $\left|\phi_{\ell_{j}}\right|^{2} \mathrm{dvol}_{g}$ weakly converge to $\mathrm{dvol}_{g}$ as $j \rightarrow \infty$. 1

If in addition $M$ has negative curvature then more is known about the geodesic flow beyond ergodicity, e.g., exponential decay of correlations, central limit theorem. In this setting Rudnick and Sarnak [39] have conjectured that the quantum limit is unique, that is, $\left|\phi_{\ell}\right|^{2} \mathrm{dvol}_{g}$ weakly converges to $\mathrm{dvol}_{g}$ along the entire sequence of eigenfunctions $\left\{\phi_{\ell}\right\}_{\ell}$. This is known as the Quantum Unique Ergodicity (QUE) conjecture. The general form of this conjecture appears to be far from being solved, however, in the special arithmetic setting where $M=\mathrm{SL}_{2}(\mathbb{Z}) \backslash \mathbb{H}$ Lindenstrauss [24] and Soundararajan [48] have shown QUE holds for a Hecke basis of eigenfunctions. Prior to these breakthroughs, this result was known to follow conditionally under

\footnotetext{
${ }^{1}$ The Quantum Ergodicity Theorem gives the stronger result that $\phi_{\ell_{j}}$ equidistributes within $S^{*} M$ as $j \rightarrow \infty$. The weak convergence $\left|\phi_{\ell_{j}}\right|^{2} \mathrm{dvol}_{g} \Rightarrow \mathrm{dvol}_{g}$ follows from projecting to configuration space.
} 
the assumption of the Generalized Riemann Hypothesis (GRH) from Watson's formula [53]. Additionally, other cases of arithmetic QUE have been established by Lindenstrauss [24] and Silberman-Venkatesh [44].

The hyperbolic surface $\mathrm{SL}_{2}(\mathbb{Z}) \backslash \mathbb{H}$ also has the structure of a complex analytic Riemannian surface and it is natural to wonder if the holomorphic analogue of QUE holds. The analogue of the eigenfunctions of $\Delta_{\mathbb{H}}$ lying in $L^{2}$ are the holomorphic modular forms, which are intrinsic to number theory. For instance a prototypical example of such a function is the modular discriminant

$$
\Delta(z)=\sum_{n \geq 1} \tau(n) e(n z)
$$

which is a weight 12 Hecke cusp form, where $\tau(n)$ is the Ramanujan $\tau$-function. In this context Lindenstrauss's method does not seem to apply. However, by combining two different approaches, one of which uses Watson's formula [53], Holowinsky and Soundararajan [11, 49, 12] established holomorphic QUE for Hecke cusp forms in the limit as the weight tends to infinity. Their result has the beautiful corollary, proved by Rudnick [38] that the zeros of all such Hecke cusp forms equidistribute with respect to hyperbolic measure within compact subsets of $\mathrm{SL}_{2}(\mathbb{Z}) \backslash \mathbb{H}$ with boundary measure zero as the weight tends to infinity. Holomorphic QUE has been established in other cases as well by Marshall [28, Nelson [32, 33], and Nelson-Pitale-Saha [34].

\subsection{Half-integral weight automorphic forms. Let}

$$
\Gamma_{0}(N)=\left\{\left(\begin{array}{ll}
a & b \\
c & d
\end{array}\right) \in \mathrm{SL}_{2}(\mathbb{Z}): c \equiv 0 \quad(\bmod N)\right\} .
$$

A weight $k$ modular form for $\Gamma_{0}(N)$ is a function $f: \mathbb{H} \rightarrow \mathbb{C}$ which, transforms in the following way

$$
f(\gamma z)=\chi(\gamma)\left(\frac{c z+d}{|c z+d|}\right)^{k} f(z), \quad \forall \gamma=\left(\begin{array}{ll}
a & b \\
c & d
\end{array}\right) \in \Gamma_{0}(N)
$$

for some character $\chi: \Gamma_{0}(N) \rightarrow S^{1}$ and satisfies a suitable growth condition at all cusps of $\Gamma_{0}(N) \backslash \mathbb{H}$. If in addition $f$ vanishes at each of the cusps of $\Gamma_{0}(N) \backslash \mathbb{H}$ we call $f$ a cusp form.

A half-integral weight Maaß cusp form for $\Gamma_{0}(4)$ is a weight $\frac{1}{2}$ cusp form that is also an $L^{2}\left(\Gamma_{0}(4) \backslash \mathbb{H}\right.$, dvol)-eigenfunction of the following differential operator

$$
\Delta_{1 / 2}=y^{2}\left(\frac{\partial^{2}}{\partial x^{2}}+\frac{\partial^{2}}{\partial y^{2}}\right)-\frac{1}{2} i y \frac{\partial}{\partial x},
$$


and $\chi$ in (1.1) is given by $\chi(\gamma)=\overline{\varepsilon_{d}}\left(\frac{c}{d}\right)$ where $\varepsilon_{d}=1$ if $d \equiv 1(\bmod 4)$ and $\varepsilon_{d}=i$ if $d \equiv-1(\bmod 4)$, and $\left(\frac{c}{d}\right)$ is Jacobi's symbol. Here $\operatorname{dvol}(z)=d x d y / y^{2}$ denotes hyperbolic measure.

For $k$ a positive integer a weight $k+\frac{1}{2}$ holomorphic modular form for $\Gamma_{0}(N)$ is a holomorphic function $g: \mathbb{H} \rightarrow \mathbb{C}$ such that $g$ is holomorphic at the cusps of $\Gamma_{0}(N) \backslash \mathbb{H}$ and $(\operatorname{Im}(z))^{\left(k+\frac{1}{2}\right) / 2} g(z)$ is a weight $k+\frac{1}{2}$ modular form with $\chi(\gamma)=\left(\frac{-1}{d}\right)^{k} \overline{\varepsilon_{d}}\left(\frac{c}{d}\right)^{2 k+1}$. These functions emerge naturally in number theory and an example of such a modular form of weight $\ell+\frac{3}{2}$ for $\Gamma_{0}(4)$ is

$$
\theta_{P}(z)=\sum_{m \in \mathbb{Z}^{3}} P(m) e\left(|m|^{2} z\right), \quad\left(e(z)=e^{2 \pi i z}\right)
$$

where $P$ is a homogeneous harmonic polynomial on $\mathbb{R}^{3}$ of degree $\ell$. Other examples of half-integral weight holomorphic forms are known to encode information on periods of integral weight forms (see [43]), and in the level aspect, assuming the Birch and Swinnerton-Dyer conjecture, their coefficients reflect the order of the TateShafarevich group of quadratic twists of elliptic curves with rank 0.

Both the holomorphic and real analytic half-integral weight modular forms play special roles in number theory. For instance, Duke [5] established the equidistribution of $\mathrm{CM}$ points on $\mathrm{SL}_{2}(\mathbb{Z}) \backslash \mathbb{H}$ by combining a formula of Maaß (which needed to be extended to the non-compact setting) along with proving a subconvexity bound for the Fourier coefficients of half-integral weight Maaß forms. This built upon the pioneering work of Iwaniec [14] who had established similar estimates for half-integral weight holomorphic modular forms, which has the application to the equidistribution of lattice points on a sphere.

In this paper we set out to investigate the analogues of QUE in the setting of holomorphic half-integral weight forms for $\Gamma_{0}(4) \backslash \mathbb{H}$, as well as for half-integral weight Maaß forms. The two cases are rather similar. For this reason, despite stating the holomorphic case first, we will give a detailed proof only in the case of Maaß forms. The rationale for our choice is that the literature dealing with half-integral weight Maaß forms is less developed and certain additional difficulties arise. We sketch the analogous argument for holomorphic half-integral weight forms in Section 9.

\subsection{Holomorphic half-integral weight forms.}

1.3.1. Setting. Write $S_{k+1 / 2}\left(\Gamma_{0}(4)\right)$ for the space of weight $k+\frac{1}{2}$ holomorphic cusp forms for $\Gamma_{0}(4)$. Every $g \in S_{k+1 / 2}\left(\Gamma_{0}(4)\right)$ has a Fourier expansion of the form

$$
g(z)=\sum_{n \geq 1} c(n) e(n z)
$$


and for odd $p$ the Hecke operator $T_{p^{2}}$ is defined on $S_{k+1 / 2}\left(\Gamma_{0}(4)\right)$ as

$$
T_{p^{2}} g(z)=\sum_{n \geq 1}\left(c\left(p^{2} n\right)+\left(\frac{(-1)^{k} n}{p}\right) p^{k-1} c(n)+p^{2 k-1} c\left(\frac{n}{p^{2}}\right)\right) e(n z) .
$$

Here we have used the convention that $c(x)=0$ if $x \notin \mathbb{Z}$. We call a half-integral weight cusp form $g$ a Hecke cusp form if $T_{p^{2}} g=\lambda_{p} g$ for all $p>2$. We recall that the half-integral weight Hecke operators vanish on the primes, thus $T_{p}=0$ for all $p$ (see Shimura [42], p. 450). We will restrict our attention to Hecke forms in what follows.

One of the main tools in understanding half-integral weight forms is the Shimura lift [42], which to a half-integral weight cusp form associates a cusp form of integer weight. Following Kohnen-Zagier [19], we focus on forms belonging to Kohnen's plus subspace on which the behavior of the Shimura lift is very well understood. Precisely the Kohnen plus space $S_{k+1 / 2}^{+}\left(\Gamma_{0}(4)\right)$ denotes the subspace of $S_{k+1 / 2}\left(\Gamma_{0}(4)\right)$ of cusp forms whose coefficients satisfy $c(n)=0$ for $(-1)^{k} n \equiv 2,3(\bmod 4)$ and has a basis consisting of simultaneous eigenfunctions of the operators $T_{p^{2}}$ for all odd $p$. We note that as $k \rightarrow \infty$ asymptotically one-third of half-integral weight Hecke cusp forms belong to the Kohnen space (since $\operatorname{dim} S_{k+1 / 2}^{+}\left(\Gamma_{0}(4)\right) \sim k / 6$ and $\operatorname{dim} S_{k+1 / 2}\left(\Gamma_{0}(4)\right) \sim k / 2$ by [20, 35] and dimension formulas)

1.3.2. Results. Compared to the case of integral weight forms, the study of QUE for half-integral weight forms presents several new difficulties. First of all, an analogue of Watson's formula [53] is not currently known to exist. Consequently, the simple conditional (on GRH) proof of QUE for the integer weight case does not carry over to the half-integral weight setting. Secondly, the unconditional techniques of Holowinsky-Soundararajan [12 are not directly applicable since they rely crucially on the multiplicativity of the coefficients. The coefficients of half-integral weight forms are not multiplicative (except at squares) and in general correspond to central values of $L$-functions, following Waldspurger [52]. In particular, one does not expect the analogue of the Riemann Hypothesis to hold for Dirichlet series built from Fourier coefficients of half-integral weight Hecke cusp forms, due to the absence of an Euler product. Our first main result establishes QUE for holomorphic half-integral weight Hecke cusp forms lying in Kohnen's plus space, assuming GRH.

Theorem 1. Assume the Generalized Riemann Hypothesis. Let $g_{k}$ be a holomorphic half-integral weight cusp form for $\Gamma_{0}(4)$ of weight $k+\frac{1}{2}$, with $k$ a positive integer. Suppose that $g_{k}$

(1) is normalized so that $\iint_{\Gamma_{0}(4) \backslash \mathbb{H}} y^{k+1 / 2}\left|g_{k}(z)\right|^{2} \operatorname{dvol}(z)=1$,

(2) lies in the Kohnen subspace,

(3) is a simultaneous eigenfunction of the Hecke operators $T_{p^{2}}, p \neq 2$. 
Let $\mathcal{D}$ be a compact subset of $\Gamma_{0}(4) \backslash \mathbb{H}$ with boundary measure zero. Then, as $k \rightarrow \infty$,

$$
\iint_{\mathcal{D}} y^{k+\frac{1}{2}}\left|g_{k}(z)\right|^{2} \operatorname{dvol}(z) \rightarrow \frac{\operatorname{vol}(\mathcal{D})}{\operatorname{vol}\left(\Gamma_{0}(4) \backslash \mathbb{H}\right)}
$$

where $\operatorname{dvol}(z)=d x d y / y^{2}$ is the hyperbolic area measure.

In the proof of Theorem 1 we specifically require the Riemann Hypothesis for $L(s, f)$ with $f \in S_{2 k}\left(\mathrm{SL}_{2}(\mathbb{Z})\right)$, all of the quadratic twists $L\left(s, f \otimes \chi_{d}\right)$, with $d$ fundamental discriminants with $(-1)^{k} d>0$ and for the symmetric square $L$-function $L\left(s, \operatorname{Sym}^{2} f\right)$.

Following the method of Rudnick [38, which is closely related to ideas of ShiffmanZelditch [41, Theorem 11 gives an immediate consequence for the distribution of zeros of holomorphic half-integral weight forms $g_{k}$ (see also [22] for a recent refinement of Rudnick's result).

Corollary 1.1. Assume the Generalized Riemann Hypothesis. Let $g_{k}$ be as in Theorem 1 . Then as $k \rightarrow \infty$ the zeros of $g_{k}$ equidistribute with respect to hyperbolic measure within compact subsets of $\Gamma_{0}(4) \backslash \mathbb{H}$ with boundary measure zero.

This means that for $g_{k}$ as in Theorem 1 we have for a compact subset $\mathcal{D} \subset \Gamma_{0}(4) \backslash \mathbb{H}$ with boundary measure zero that

$$
\sum_{\substack{g_{k}(\varrho)=0 \\ \varrho \in \mathcal{D}}} 1=\frac{k}{2} \cdot \frac{\operatorname{vol}(\mathcal{D})}{\operatorname{vol}\left(\Gamma_{0}(4) \backslash \mathbb{H}\right)}+o(k)
$$

as $k \rightarrow \infty$, with the zeros counted with multiplicity.

\subsection{Half-integral weight Maaß forms.}

1.4.1. Setting. Let $V$ denote the space generated by the half-integral weight Maaß cusp forms for $\Gamma_{0}(4)$. Every half-integral weight Maaß cusp form, $g$, with eigenvalue $\lambda=-\left(\frac{1}{4}+t^{2}\right)$ has a Fourier expansion at the cusp at $\infty$ of the following form

$$
g(z)=\sum_{n \neq 0} b_{g, \infty}(n) W_{\frac{1}{4} \operatorname{sgn}(n), i t}(4 \pi|n| y) e(n x), \quad(z=x+i y)
$$

where $W$ is the Whittaker function. We will restrict our attention to half-integral weight Maaß cusp forms which are in addition eigenfunctions of the Hecke operators $T_{p^{2}}: V \rightarrow V, p$ an odd prime. For $p>2$ the action of a Hecke operator $T_{p^{2}}$ on a Maaß cusp form $g$ is given explicitly by

$$
T_{p^{2}} g(z)=\sum_{n \neq 0} c(n) W_{\frac{1}{4} \operatorname{sgn}(n), i t}(4 \pi|n| y) e(n x),
$$


where

$$
c(n)=p b_{g, \infty}\left(n p^{2}\right)+p^{-1 / 2}\left(\frac{n}{p}\right) b_{g, \infty}(n)+p^{-1} b_{g, \infty}\left(\frac{n}{p^{2}}\right)
$$

(see Theorem 1.7 of Shimura [42]). The operators $T_{p^{2}}$ are self-adjoint and commute with each other as well as with $\Delta_{1 / 2}$ (see Theorem 1 of [20] for the holomorphic setting and Proposition 1.4 of [17] for the real analytic one). As before, we will only consider Hecke Maaß cusp forms, which are half-integral weight Maaß forms which are eigenfunctions of $T_{p}^{2}$, for $p$ odd.

Similarly to the holomorphic case, we have at our disposal the Shimura lift, which is well understood on the Kohnen subspace. In particular, this associates a halfintegral weight form, $g$, with $b_{g, \infty}(n)=0$ for $n \equiv 2,3(\bmod 4)$ with a weight 0 Hecke Maaß cusp form for $\mathrm{SL}_{2}(\mathbb{Z})$ (see Katok-Sarnak [17]). The Kohnen space $V^{+}$is the subspace generated by such forms.

1.4.2. Results. Before stating our results for Maaß forms we highlight that it might be possible to apply the ergodic techniques of Lindenstrauss in the setting of halfintegral weight Maaß forms. Lindenstrauss's method would not however rule out the possibility of escape of mass into the cusps. In our next theorem we establish, conditionally on GRH, QUE for half-integral weight Hecke Maaß cusp forms and also eliminate the possibility of escape of mass. It is also worth pointing out that our result can be made effective, and yields a slow rate of convergence to equidistribution. On the other hand making Lindenstrauss's result effective remains a challenging open problem.

Theorem 2. Assume the Generalized Riemann Hypothesis. Let $g_{j}$ be a basis of the Kohnen space $V^{+}$, such that $\iint_{\Gamma_{0}(4) \backslash \mathbb{H}}\left|g_{j}(z)\right|^{2} \operatorname{dvol}(z)=1$ for all $j$ and each $g_{j}$ is a simultaneous eigenfunction of the Hecke operators $T_{p^{2}}$ for all $p>2$ as well as of $\Delta_{1 / 2}$ with $\Delta_{1 / 2} g_{j}=-\left(\frac{1}{4}+t_{j}^{2}\right) g_{j}$. Let $\mathcal{D}$ be a compact subset of $\Gamma_{0}(4) \backslash \mathbb{H}$ with boundary measure zero. Then, as $\left|t_{j}\right| \rightarrow \infty$,

$$
\iint_{\mathcal{D}}\left|g_{j}(z)\right|^{2} \operatorname{dvol}(z) \rightarrow \frac{\operatorname{vol}(\mathcal{D})}{\operatorname{vol}\left(\Gamma_{0}(4) \backslash \mathbb{H}\right)}
$$

where $\operatorname{dvol}(z)=d x d y / y^{2}$ is the hyperbolic area measure.

In the proof of Theorem 2 we specifically require the Riemann Hypothesis for $L$ functions of weight 0 Hecke-Maaß eigencuspforms for the full modular group, all of their quadratic twists and the symmetric square $L$-function $L\left(s, \operatorname{Sym}^{2} f\right)$ with $f$ a weight 0 Hecke-Maaß eigencuspform for $\mathrm{SL}_{2}(\mathbb{Z})$. The problem of equidistribution of half-integral weight Eisenstein series has been recently addressed by Petridis-RaulfRisager [36], where they prove QUE for half-integral weight Eisenstein series under the assumption of a subconvexity bound for a multiple Dirichlet series. Establishing 
such a subconvexity bound is still open, apparently even under GRH. In the weight 0 case Luo-Sarnak [26] and Jakobson [16] have unconditionally established QUE for the Eisenstein series.

1.5. Comments on the proof and further work. Our proof takes elements from Holowinsky's [11] unconditional argument for holomorphic integer weight forms and Soundararajan's conditional upper bounds for moments of $L$-functions [47. Similarly to Holowinsky we have to estimate a certain main term and an off-diagonal term consisting of coefficients of half-integral weight forms. Holowinsky's treatment of the off-diagonal is based on sieve estimates, while ours is based on Soundararajan's work on moments. It is interesting to notice that Soundararajan's proof could have been carried out also in the multiplicative setting where it would deliver the corresponding sieve bound. Similarly to Holowinsky's treatment [11] we also experience considerable difficulties with estimating the main term. In our case the estimation of the main term corresponds to averaging a Dirichlet polynomial of length $X^{2}$ over quadratic characters of conductor $\asymp X$. This is a notoriously difficult scenario because an application of the Poisson summation gives back a sum of the same length. We explain how this significant obstruction is resolved in our work in the next section.

In the case of weight zero level 1 Hecke Maaß forms Watson's formula and the Generalized Lindelöf Hypothesis imply an upper bound on the rate of convergence in QUE of size $\ll \lambda^{-1 / 4+\varepsilon}$. Currently, no effective rate is unconditionally known in this setting, but has been worked out for the holomorphic case, i.e. Hecke cusp forms for $\mathrm{SL}_{2}(\mathbb{Z})$, in $[22$, where the rate obtained is a small negative power of the logarithm of the weight. It also emerges from an inspection of our proof that in the half-integral case we only get a bound of $\ll(\log \lambda)^{-\delta}$, for some $\delta>0$, on the rate of convergence to uniform distribution. It would be very interesting to obtain a bound of size $\ll \lambda^{-\delta}$ for some $\delta>0$.

Finally in a future paper we plan to address the problem of Quantum Ergodicity for half-integral weight forms, that is, to show that the mass of almost all holomorphic forms in the Kohnen plus space tends to equidistribution as the weight increases (it is also likely that the result extends to Maaß forms but for simplicity we have decided not to work out this case). This means that at least as far holomorphic forms go, the situation in the half-integral case is qualitatively the same as in the integer weight weight case before the breakthroughs of Holowinsky-Soundararajan. It will be therefore very interesting to see if unconditional results are also possible to obtain.

\section{Overview of the ARGUMENT}

2.1. Reduction to sums of Fourier coefficients. For simplicity write $g=g_{j}$ and $t=t_{j}$ so that $\Delta_{1 / 2} g_{j}=-\left(\frac{1}{4}+t^{2}\right) g_{j}$. Note that $t \in \mathbb{R}$ since there are no half-integral 
Maaß forms in $V^{+}$with exceptional eigenvalues? 2 . Since we will take $|t| \rightarrow \infty$ we assume outright that $|t|>100$. By an approximation argument it suffices to show that for a test function $\Psi(z) \in C_{c}^{\infty}\left(\Gamma_{0}(4) \backslash \mathbb{H}\right)$

$$
\iint_{\Gamma_{0}(4) \backslash \mathbb{H}} \Psi(z)|g(z)|^{2} \frac{d x d y}{y^{2}} \rightarrow \iint_{\Gamma_{0}(4) \backslash \mathbb{H}} \Psi(z) \frac{d x d y}{y^{2}} \quad(|t| \rightarrow \infty) .
$$

For $\Psi(z) \in C_{c}^{\infty}\left(\Gamma_{0}(4) \backslash \mathbb{H}\right)$ one has $\Psi(z+1)=\Psi(z)$ and we can define $\widetilde{\Psi}(z)$ to be the extension of $\Psi$ to $\mathbb{H}$ by $\Gamma_{\infty}$-periodicity. Additionally, we see that $\widetilde{\Psi}_{y}(x):=\widetilde{\Psi}(x+i y)$ has a Fourier expansion. Using this expansion and adapting an argument of Luo and Sarnak, (see Section 4 of [26], but some care must be taken at the elliptic points of $\left.\Gamma_{0}(4) \backslash \mathbb{H}\right)$, one can expand $\Psi(z)$ into a sum of Poincaré series

$$
P_{h, \ell}(z)=\sum_{\gamma \in \Gamma_{\infty} \backslash \Gamma_{0}(4)} h(\operatorname{Im}(\gamma z)) e(\ell \operatorname{Re}(\gamma z))
$$

where $h(y)$ is the $\ell$ th Fourier coefficient of $\widetilde{\Psi}_{y}(x)$. Thus, we see that Theorem 2 follows from estimates for the diagonal term $\ell=0$ (here $P_{h, 0}(z)=E(z \mid h)$ is the incomplete Eisenstein series)

$$
\begin{aligned}
\iint_{\Gamma_{0}(4) \backslash \mathbb{H}} E(z \mid h)|g(z)|^{2} \frac{d x d y}{y^{2}} & =\frac{1}{\operatorname{vol}\left(\Gamma_{0}(4) \backslash \mathbb{H}\right)} \iint_{\Gamma_{0}(4) \backslash \mathbb{H}} E(z \mid h) \frac{d x d y}{y^{2}}+o(1) \quad(|t| \rightarrow \infty) \\
& =\frac{1}{\operatorname{vol}\left(\Gamma_{0}(4) \backslash \mathbb{H}\right)} \int_{0}^{\infty} h(y) \frac{d y}{y^{2}}+o(1)
\end{aligned}
$$

along with the bound for the off-diagonal terms, $\ell \neq 0$,

$$
\iint_{\Gamma_{0}(4) \backslash \mathbb{H}} P_{h, \ell}(z)|g(z)|^{2} \frac{d x d y}{y^{2}}=\frac{1}{\operatorname{vol}\left(\Gamma_{0}(4) \backslash \mathbb{H}\right)} \iint_{\Gamma_{0}(4) \backslash \mathbb{H}} P_{h, \ell}(z) \frac{d x d y}{y^{2}}+o(1)=o(1) \quad(|t| \rightarrow \infty) .
$$

The left-hand sides of both equations above are estimated in terms of the sums of Fourier coefficients of $g(z)$. By the unfolding technique it is easy to see that

$$
\iint_{\Gamma_{0}(4) \backslash \mathbb{H}} E(z \mid h)|g(z)|^{2} \frac{d x d y}{y^{2}}=\sum_{n \neq 0}\left|b_{g, \infty}(n)\right|^{2} \int_{0}^{\infty} W_{\frac{1}{4} \operatorname{sgn}(n), i t}(4 \pi y|n|)^{2} h(y) \frac{d y}{y^{2}}
$$

\footnotetext{
${ }^{2}$ This follows from the fact that the Shimura lift of a Maaß form $g \in V^{+}$with eigenvalue $-\left(\frac{1}{4}+t^{2}\right)$ is a Maaß cusp form for $\mathrm{SL}_{2}(\mathbb{Z})$ with eigenvalue $-\left(\frac{1}{4}+(2 t)^{2}\right)$. Since $\Delta$ on $\mathrm{SL}_{2}(\mathbb{Z})$ has no exceptional eigenvalues it follows that neither does $V^{+}(4)$
} 
and for $\ell \neq 0$

$$
\begin{aligned}
& \iint_{\Gamma_{0}(4) \backslash \mathbb{H}} P_{h, \ell}(z)|g(z)|^{2} \frac{d x d y}{y^{2}} \\
& =\sum_{n \neq 0} b_{g, \infty}(n) b_{g, \infty}(n+\ell) \int_{0}^{\infty} W_{\frac{1}{4} \operatorname{sgn}(n), i t}(4 \pi|n| y) W_{\frac{1}{4} \operatorname{sgn}(n+\ell), i t}(4 \pi|n+\ell| y) h(y) \frac{d y}{y^{2}} .
\end{aligned}
$$

Thus the problem is reduced to estimating (2.1) and (2.2) as $|t| \rightarrow \infty$.

2.2. Estimating the sums of Fourier coefficients. At this point we appeal to a consequence of a Waldspurger type formula, obtained in the setting of half-integral weight Maaß forms by Baruch and Mao [1] (with related works by Biró [2] and KatokSarnak [17]). It follows from this formula that if $g \in V^{+}$is a half-integral weight Hecke Maaß cusp form then for $d$ a fundamental discriminant and $\delta \geq 1$ any integer,

$$
\left|b_{g, \infty}\left(d \delta^{2}\right)\right| \ll_{\varepsilon} \frac{1}{\sqrt{|n|}}\left(\frac{L\left(\frac{1}{2}, f \otimes \chi_{d}\right)}{L\left(1, \operatorname{Sym}^{2} f\right)}\right)^{1 / 2} \cdot \delta^{7 / 64+\varepsilon} \cdot|t|^{-\operatorname{sgn}(n) / 4} \cdot e^{\pi|t| / 2}, n=d \delta^{2}
$$

where $f$ is the Shimura lift of $g$ and in particular $f$ is a Hecke-Maaß cusp form for $\mathrm{SL}_{2}(\mathbb{Z})$ (see Section 10). The exponent $7 / 64$ is the exponent towards the Ramanujan conjecture obtained by Kim and Sarnak [18]. In fact any exponent $<\frac{1}{2}$ would do (with some additional work). Using the triangle inequality, this reduces the problem of estimating (2.2) to showing that for $\ell \neq 0$,

$$
\frac{1}{L\left(1, \mathrm{Sym}^{2} f\right)} \sum_{\substack{d_{1} \delta_{1}^{2}-d_{2} \delta_{2}^{2}=\ell \\|t| \leq d_{1} \delta_{1}^{2} \leq 2|t|}} L\left(\frac{1}{2}, f \otimes \chi_{d_{1}}\right)^{1 / 2} L\left(\frac{1}{2}, f \otimes \chi_{d_{2}}\right)^{1 / 2}=o(|t|)
$$

where the summation is over fundamental discriminants $d_{1}, d_{2}$ and integers $\delta_{1}, \delta_{2}$. On the other hand the estimation of the main term (2.1) is, through the use of an exact Waldspurger formula, essentially equivalent to showing that

$$
\frac{1}{L\left(1, \operatorname{Sym}^{2} f\right)} \sum_{|t| \leq d \leq 2|t|} L\left(\frac{1}{2}, f \otimes \chi_{d}\right) \sim c|t|
$$

for some constant $c>0$, where the summation is over fundamental discriminants $d$ (although we note that while $(2.3)$ is useful to explain the technical issues that arise, we never deal with (2.3) directly but rather prefer to phrase the problem of estimating (2.1) in terms of the coefficients $\left.\left|b_{g, \infty}(d)\right|^{2}\right)$.

We obtain the first bound as a consequence of Soundararajan's work on moments of $L$-functions (in fact gaining a small logarithmic saving). That such a saving 
is possible is suggested by the following "Sato-Tate" property of the coefficients $L\left(\frac{1}{2}, f \otimes \chi_{d}\right)$,

$$
\sum_{X \leq|d| \leq 2 X} L\left(\frac{1}{2}, f \otimes \chi_{d}\right)^{1 / 2}=o(X)
$$

which is analogous to

$$
\sum_{X \leq n \leq 2 X}\left|\lambda_{f}(n)\right|=o(X)
$$

where $\lambda_{f}(n)$ are the Hecke eigenvalues of $f$. We remark that for holomorphic forms the first bound is now known unconditionally [37], while the second has been known for quite some time [7], and played a critical role in Holowinsky's work [11.

Let us now turn our attention to the second estimate (2.3) that we need to establish QUE. The main difficulty here is the following: since $d \asymp|t|$ and $f$ has also spectral parameter $\asymp|t|$ the length of the Dirichlet polynomial approximating $L\left(\frac{1}{2}, f \otimes \chi_{d}\right)$ is $|t|^{2}$. Therefore applying Poisson summation for quadratic characters with conductor $\asymp|t|$ will return a sum of the same length. This means that we are confronted with the notorious "deadlock situation" discussed for example by Munshi in [31], but in any case well recognized by experts.

We overcome the deadlock situation by using once again another idea of Holowinsky. The starting point is to notice that $L\left(\frac{1}{2}, f \otimes \chi_{d}\right)$ is proportional to $\left|b_{g, \infty}(d)\right|^{2}$ so instead of directly evaluating the moment (2.3) we head back to the expression for this moment in terms of the Rankin-Selberg integral (2.1). In order to evaluate the Rankin-Selberg convolution we consider the following expression

$$
\iint_{\Gamma_{0}(4) \backslash \mathbb{H}}|g(z)|^{2} E(z \mid h) E^{Y}(z \mid k) \operatorname{dvol}(z)
$$

where

$$
E^{Y}(z \mid k)=\sum_{\gamma \in \Gamma_{\infty} \backslash \Gamma_{0}(4)} k(Y \cdot \operatorname{Im}(\gamma z)) .
$$

with $k$ a smooth function. Evaluating the above in two different ways shows that the summation in (2.3) can be extended by a factor of $Y$ provided that we have a saving of say, $Y^{100}$, in the shifted convolution problem (2.2). This allows us to extend the length of summation in (2.3) by a small power of $\log |t|$, and consequently after applying Poisson summation (which now returns a sum slightly shorter than $|t|$ ) and using once again the work of Soundararajan [47] on moments, we obtain an asymptotic estimate for (2.3). In the actual proof we phrase these details slightly differently by establishing a "convexity bound" for the Dirichlet series with coefficients $\left|b_{g, \infty}(n)\right|^{2}$ which then gives an asymptotic evaluation of the average of $\left|b_{g, \infty}(n)\right|^{2}$ when slightly more than $|t|$ terms are added up. 
It is worthwhile to point out that in similar problems involving the second or fourth moment of $L$-functions (as considered by Soundararajan-Young [46]) one benefits from the presence of a power greater than the first. This feature is not present in our moment problem, however. Finally we notice that if we could obtain unconditionally a power saving in the shifted convolution problem (2.2), then we could extend the length of summation in the first moment by a small power of $|t|$ and then evaluate this first moment unconditionally. This would lead to an unconditional proof of QUE for half-integral weight forms, with a power-saving in the rate of convergence. A powersaving in the shifted convolution problem (2.2) seems to however remain far out of reach, in particular due to the sum being averaged in a range which corresponds to $|t|$ where $t$ is the spectral parameter.

\section{Estimates For CharaCter sums}

The results on moments of $L$-functions which appear in the next section depend on a character sum estimate which we obtain below.

Proposition 3.1. Let $F$ be a Schwartz function with $\widehat{F}$ compactly supported in $(-A, A)$ for some fixed $A>0$. Also, let $\ell$ be an integer and $a, b$ be integers such that $1 \leq a, b \leq X^{\varepsilon}$. Suppose $1 \leq r, s \leq X^{\varepsilon}$ are odd integers with $(a b, r s)=1$. Write $r=r_{0} q^{2}$ and $s=s_{0} w^{2}$ where $r_{0}, s_{0}$ are square-free and let

$$
\mathcal{M}\left(r_{0}, q, w, a, b, \ell\right)=\sum_{\substack{d_{1}\left|q, d_{2}\right| w \\\left(d_{1} d_{2}, r_{0}\right)=1 \\\left(a d_{1}, b d_{2}\right) \mid \ell}} \frac{\mu\left(d_{1}\right) \mu\left(d_{2}\right)}{\left[d_{1}, d_{2}\right]} \frac{c_{r_{0}}\left(\ell /\left(a d_{1}, b d_{2}\right)\right)}{r_{0}}
$$

where $c_{r}(\ell):=\sum_{(a, r)=1} e(a \ell / r)$ is a Ramanujan sum. Then, for all $X$ large enough with respect to $A$, if $s_{0}=r_{0}$

$$
\sum_{\substack{m, n \\ a m=b n+\ell}}\left(\frac{m}{r}\right)\left(\frac{n}{s}\right) F\left(\frac{a m}{X}\right)=\left(\frac{a b /(a, b)^{2}}{r_{0}}\right) \widehat{F}(0) \frac{X}{[a, b]} \cdot \mathcal{M}\left(r_{0}, q, w, a, b, \ell\right)
$$

and if $r_{0} \neq s_{0}$

$$
\sum_{\substack{m, n \\ a m=b n+\ell}}\left(\frac{m}{r}\right)\left(\frac{n}{s}\right) F\left(\frac{a m}{X}\right)=0 .
$$

Remark 1. In the simple case where $a=b=1, \ell=0$ and $s=1$ this result reduces to the estimate

$$
\sum_{m}\left(\frac{m}{r}\right) F\left(\frac{m}{X}\right)= \begin{cases}\widehat{F}(0) \varphi(r) \cdot \frac{X}{r} & \text { if } r=\square \\ 0 & \text { otherwise }\end{cases}
$$

which we will also use later. 
Additionally, it is easily seen from the proof that if $F$ is only a Schwartz function then the results of Proposition 3.1 remain true up to an additional error term of $O_{A}\left(X^{-A}\right)$. The proof of Proposition 3.1 depends on the following version of Poisson summation, which we recall here.

Lemma 3.1. Let $F$ be a Schwartz function. Suppose that $(d, r)=1$. Then,

$$
\sum_{n \equiv t}\left(\frac{n}{r}\right) F(n)=\frac{1}{d r}\left(\frac{d}{r}\right) \sum_{k} \widehat{F}\left(\frac{k}{d r}\right) e\left(\frac{t k \bar{r}}{d}\right) \tau_{k}(r)
$$

where

$$
\tau_{k}(n):=\sum_{b}\left(\frac{b}{n}\right) e\left(\frac{k b}{n}\right)
$$

and

$$
\widehat{F}(x):=\int_{\mathbb{R}} F(u) e(-x u) d u
$$

is the Fourier transform of $F$.

Proof. We have

$$
\sum_{n \equiv t}\left(\frac{n}{r}\right) F(n)=\sum_{b}\left(\frac{b}{r}\right) \sum_{\substack{n \equiv b \\ n \equiv t \\ n \equiv t}(\bmod r)} F(n) .
$$

Since $(r, d)=1$, the congruence condition can be re-written as $n \equiv b d \bar{d}+t r \bar{r}$ $(\bmod r d)$. This way we re-write the inner sum over $n$ as

$$
\sum_{n \equiv b d \bar{d}+t r \bar{r}} F(n) .
$$

By Poisson summation

$$
\sum_{n \equiv b d \bar{d}+t r \bar{r}} F(n)=\frac{1}{r d} \sum_{k} \widehat{F}\left(\frac{k}{r d}\right) e\left(\frac{k b \bar{d}}{r}+\frac{k t \bar{r}}{d}\right)
$$

and the claim follows.

We are now ready to prove the proposition.

Proof of Proposition 3.1. Dividing the linear condition $a m=b n+\ell$ by $(a, b)$ we see that we need to have $(a, b) \mid \ell$ and that upon substituting $a /(a, b)$ for $a, b /(a, b)$ for $b$, $\ell /(a, b)$ for $\ell$ and finally $X /(a, b)$ for $X$, we see that we can as well assume that $a$ and $b$ are co-prime from the outset, which we will do now.

Consider first the case where both $r$ and $s$ are square-free. Write $r=d r^{\star}$ and $s=d s^{\star}$ with $d=(r, s)$. Since $r$ was assumed to be square-free, $\left(d, r^{\star}\right)=1$. Given $t$ 
$(\bmod d)$ if we require that $m \equiv t(\bmod d)$ then the condition $m a=n b+\ell$ implies that $\bar{b}(t a-\ell) \equiv n(\bmod d)$, with $\bar{b}$ the modular inverse of $b(\bmod d)$. Therefore,

$$
\sum_{\substack{m, n \\ a m=b n+\ell}}\left(\frac{m}{r}\right)\left(\frac{n}{s}\right) F\left(\frac{a m}{X}\right)=\sum_{t}\left(\frac{t \bar{b}(t a-\ell)}{d}\right) \sum_{\substack{m \equiv t \\(\bmod d) \\ m a=n b+\ell}}\left(\frac{m}{r^{\star}}\right)\left(\frac{n}{s^{\star}}\right) F\left(\frac{a m}{X}\right) .
$$

Moreover given $u\left(\bmod s^{\star}\right)$ we see that summing over $m, n$ such that $n \equiv u\left(\bmod s^{\star}\right)$ and $m a-n b=\ell$ is the same as summing over $m, n$ such that $n \equiv u\left(\bmod s^{\star}\right)$ and $m a-\ell \equiv u b\left(\bmod b s^{\star}\right)$. With this in mind we re-write the inner sum over $m$ as

$$
\begin{aligned}
& S:=\sum_{u}\left(\frac{u}{s^{\star}}\right) \sum_{\substack{m \equiv t \\
m a-\ell \equiv u b}}\left(\frac{m}{\bmod ^{\star}\left(\bmod b s^{\star}\right)}\right) F\left(\frac{m a}{X}\right) \\
& =\sum_{u}\left(\frac{u}{s^{\star}}\right) \sum_{m \equiv t}\left(\frac{m}{r^{\star}}\right) \cdot \frac{1}{b s^{\star}} \sum_{0 \leq v<b s^{\star}} e\left(\frac{v(m a-u b-\ell)}{b s^{\star}}\right) F\left(\frac{m a}{X}\right) \\
& =\frac{1}{b s^{\star}} \sum_{0 \leq v<b s^{\star}} e\left(\frac{-v \ell}{b s^{\star}}\right) \sum_{u}\left(\frac{u}{s^{\star}}\right) e\left(-\frac{v u}{s^{\star}}\right) \sum_{m \equiv t}\left(\frac{m}{(\bmod d)}\left(\frac{m}{r^{\star}}\right) e\left(\frac{v m a}{b s^{\star}}\right) F\left(\frac{m a}{X}\right) .\right.
\end{aligned}
$$

Applying Lemma 3.1 we get

$$
\sum_{m \equiv t}\left(\frac{m}{r^{\star}}\right) e\left(\frac{a v m}{b s^{\star}}\right) F\left(\frac{m a}{X}\right)=\frac{X}{a r^{\star} d}\left(\frac{d}{r^{\star}}\right) \sum_{k} \widehat{F}\left(\frac{X}{a}\left(\frac{k}{d r^{\star}}-\frac{v a}{b s^{\star}}\right)\right) e\left(\frac{k t \overline{r^{\star}}}{d}\right) \tau_{k}\left(r^{\star}\right)
$$

where we have used the assumption $\left(d, r^{\star}\right)=1$. Since $d, a, b, r^{\star}, s^{\star}<X^{\varepsilon}$ and $\widehat{F}$ is compactly supported in $(-A, A)$ the above term is equal to 0 for all large enough $X$ unless $k b s^{\star}=v a d r^{\star}$. Since $s$ is square-free $\left(s^{\star}, d\right)=1$ so $\left(b s^{\star}, a d r^{\star}\right)=1$. Therefore $b s^{\star} \mid v$, but since $0 \leq v<b s^{\star}$ this implies that $v=0$, and hence that also $k=0$. Therefore

$$
S=\frac{X}{a b r^{\star} s^{\star} d} \widehat{F}(0)\left(\frac{d}{r^{\star}}\right) \sum_{u}\left(\frac{u}{s^{\star}}\right) \sum_{u^{\prime}}\left(\frac{u^{\prime}}{r^{\star}}\right)
$$

The main term is clearly zero unless $s^{\star}=r^{\star}=1$, which implies that $r=s$. Hence,

$$
\sum_{\substack{m, n \\ a m=b n+\ell}}\left(\frac{m}{r}\right)\left(\frac{n}{s}\right) F\left(\frac{a m}{X}\right)=\delta_{r=s} \frac{X}{a b r} \widehat{F}(0) \sum_{t}\left(\frac{t \bar{b}(t a-\ell)}{r}\right) .
$$

To complete the proof in the square-free case we now estimate the sum on the right-hand side of (3.1). First observe that

$$
\frac{1}{r} \sum_{0 \leq t<r} e\left(\frac{-t \ell}{r}\right) \tau_{a t}(r) \overline{\tau_{b t}(r)}=\sum_{t}\left(\frac{\bar{b} t(a t-\ell)}{r}\right) .
$$


Next, for odd square-free $r$ a little calculation using Gauss sums and quadratic reciprocity gives

$$
\tau_{v}(r)=\left(\frac{1+i}{2}+\left(\frac{-1}{r}\right) \frac{1-i}{2}\right)\left(\frac{v}{r}\right) \sqrt{r} .
$$

In particular $\tau_{v}(r)=0$ unless $(v, r)=1$, and, if $(v, r)=1$ then

$$
\tau_{a v}(r) \overline{\tau_{b v}(r)}=\left(\frac{a v}{r}\right) \sqrt{r}\left(\frac{b v}{r}\right) \sqrt{r}=\left(\frac{a b}{r}\right) r .
$$

Thus,

$$
\frac{1}{r} \sum_{0 \leq t<r} e\left(\frac{-t \ell}{r}\right) \tau_{a t}(r) \overline{\tau_{b t}(r)}=\left(\frac{a b}{r}\right) c_{r}(\ell)
$$

where $c_{r}(\ell)$ is a Ramanujan sum. Combining this with (3.1) and (3.2) completes the proof for square-free $r, s$.

Write now $r=r_{0} q^{2}$ and and $s=s_{0} w^{2}$ with $r_{0}$ and $s_{0}$ square-free. Then we can re-write the sum as

$$
\sum_{\substack{m, n \\ a m=b n+\ell \\ m, q)=(n, w)=1}}\left(\frac{m}{r_{0}}\right)\left(\frac{n}{s_{0}}\right) F\left(\frac{a m}{X}\right)
$$

Introducing Möbius inversion this is equal to

$$
\sum_{\substack{d_{1}\left|q, d_{2}\right| w \\\left(d_{1}, r_{0}\right)=\left(d_{2}, s_{0}\right)=1}} \mu\left(d_{1}\right) \mu\left(d_{2}\right)\left(\frac{d_{1}}{r_{0}}\right)\left(\frac{d_{2}}{s_{0}}\right) \sum_{\substack{m, n \\ a d_{1} m=b d_{2} n+\ell}}\left(\frac{m}{r_{0}}\right)\left(\frac{n}{s_{0}}\right) F\left(\frac{a d_{1} m}{X}\right) .
$$

By the result for square-free $r$ and $s$, this equals zero unless $r_{0}=s_{0}$ and in this case this equals

$$
\begin{aligned}
& \hat{F}(0) \frac{X}{[a, b]} \sum_{\begin{array}{c}
d_{1}\left|q, d_{2}\right| w \\
\left(d_{1} d_{2}, r_{0}\right)=1 \\
\left(a d_{1}, b d_{2}\right) \mid \ell
\end{array}} \frac{\mu\left(d_{1}\right) \mu\left(d_{2}\right)}{\left[d_{1}, d_{2}\right]}\left(\frac{d_{1} d_{2}}{r_{0}}\right) \cdot\left(\frac{a d_{1} b d_{2} /\left(a d_{1}, b d_{2}\right)^{2}}{r_{0}}\right) \frac{c_{r_{0}}\left(\ell /\left(a d_{1}, b d_{2}\right)\right)}{r_{0}} \\
& =\left(\frac{a b /(a, b)^{2}}{r_{0}}\right) \hat{F}(0) \frac{X}{[a, b]} \cdot \sum_{\substack{d_{1}\left|q, d_{2}\right| w \\
\left(d_{1} d_{2}, r_{0}\right)=1 \\
\left(a d_{1}, b d_{2}\right) \mid \ell}} \frac{\mu\left(d_{1}\right) \mu\left(d_{2}\right)}{\left[d_{1}, d_{2}\right]} \frac{c_{r_{0}}\left(\ell /\left(a d_{1}, b d_{2}\right)\right)}{r_{0}},
\end{aligned}
$$

since $\left(a d_{1}, b d_{2}\right)=(a, b)\left(d_{1}, d_{2}\right)$ because $d_{1} \mid r$ and $d_{2} \mid s$ and $(r s, a b)=1$ by assumption. 


\section{A moment calculation}

The main result of this section is an estimate for moments of a short Dirichlet polynomial, presented in Lemma 4.1 below. This will be the crucial ingredient in our bound for

$$
\sum_{\substack{d_{1}, d_{2} \\ a \mid d_{1} \leq X \\ a d_{1}=b d_{2}+\ell}} L\left(\frac{1}{2}, f \otimes \chi_{d_{1}}\right)^{1 / 2} L\left(\frac{1}{2}, f \otimes \chi_{d_{2}}\right)^{1 / 2}
$$

obtained in the next section, where the summation is over fundamental discriminants $d_{1}, d_{2}$ and $f$ is an even, arithmetically normalized, Hecke-Maaß cusp form for $\mathrm{SL}_{2}(\mathbb{Z})$.

Lemma 4.1. Let $x \leq X^{\varepsilon / k}$ for some small $\varepsilon>0$ and $1 \leq a, b,|\ell| \leq(\log X)^{100}$. For arbitrary real coefficients $a(p)$ such that $|a(p)| \leq p^{1 / 2-\delta}$ for some $\delta>0$ we have

$$
\begin{aligned}
& \sum_{\substack{d_{1}, d_{2} \\
a \mid d_{1} \leq \leq X \\
a d_{1}=b d_{2}+\ell}}\left(\sum_{\substack{2<p \leq x \\
p \nmid a b}} \frac{a(p)\left(\chi_{d_{1}}(p)+\chi_{d_{2}}(p)\right)}{\sqrt{p}}\right)^{2 k} \\
& \ll \frac{X}{[a, b]} \cdot \frac{(2 k) !}{2^{k} k !}\left(2 \sum_{p \leq x} \frac{a(p)^{2}}{p}+O\left(1+\sum_{p \mid \ell} \frac{a(p)^{2}}{p}\right)\right)^{k}
\end{aligned}
$$

provided that $x$ is chosen so that $\sum_{p \leq x} a(p)^{2} / p \gg 1+\sum_{p \mid \ell} a(p)^{2} / p$ and where the sum is taken over fundamental discriminants $d_{1}, d_{2}$.

The proof of Lemma 4.1 depends on the following lemma, which uses the character sum estimate obtained in the previous section.

Lemma 4.2. Let $F$ be a Schwartz function with $\widehat{F}$ compactly supported in $(-10,10)$, and let $a(p)$ be a sequence of real coefficients such that $|a(p)| \leq p^{1 / 2-\delta}$ for some $\delta>0$. Suppose that $1 \leq a, b,|\ell| \leq(\log X)^{100}$. Let

$$
B(x)=\frac{1}{2} \sum_{p \leq x} \frac{a(p)^{2}}{p} \text { and } A(\ell):=1+\sum_{p \mid \ell} \frac{a(p)^{2}}{p}
$$

Then, for $k, j \geq 0$ and $x$ such that

(1) $x^{k+j} \leq X^{\varepsilon}$ for some small $\varepsilon>0$,

(2) $B(x) \gg A(\ell)$, 
we have,

$$
\begin{aligned}
& \sum_{\substack{m, n \\
a m=b n+\ell}}\left(\sum_{\substack{2<p \leq x \\
p \nmid a b}} \frac{a(p)\left(\frac{m}{p}\right)}{\sqrt{p}}\right)^{k}\left(\sum_{\substack{2<p \leq x \\
p \nmid a b}} \frac{a(p)\left(\frac{n}{p}\right)}{\sqrt{p}}\right)^{j} F\left(\frac{a m}{X}\right) \ll \\
& \ll \mathbf{1}_{2 \mid k-j} \cdot \widehat{F}(0) \frac{X}{[a, b]} \cdot C(k) C(j) \cdot(2 B(x)+O(A(\ell)))^{(k+j) / 2-\eta(k, j)} \cdot A(\ell)^{\eta(k, j)}
\end{aligned}
$$

where $C(k)=k ! /\left(\lfloor k / 2\rfloor ! 2^{k / 2}\right)$ and $\eta=\eta(k, j)$ equals 0 if $k \equiv j \equiv 0(\bmod 2)$ and $\eta=1$ if $k \equiv j \equiv 1(\bmod 2)$.

We will prove Lemma 4.2 and Lemma 4.1 shortly, but beforehand we state and prove the following fairly standard lemma (see for example [47, Lemma 3]) that will also be required later on.

Lemma 4.3. Let $J>0$ be given. Let $F$ be a Schwartz function such that $\widehat{F}$ has compact support in $(-10,10)$. Suppose $k_{1}, \ldots, k_{J}$ are non-negative integers such that $x^{k_{1}+\cdots+k_{J}} \leq X^{\varepsilon}$. If the intervals $I_{j}$ are disjoint and all contained in $[1, x]$, then, for real coefficients $a(p)$,

$$
\left|\sum_{m} \prod_{j \leq J}\left(\sum_{p \in I_{j}} \frac{a(p)\left(\frac{m}{p}\right)}{\sqrt{p}}\right)^{k_{j}} F\left(\frac{m}{X}\right)\right| \leq \widehat{F}(0) X \prod_{j \leq J}\left(\widetilde{C}\left(k_{j}\right) \cdot\left(\sum_{p \in I_{j}} \frac{a(p)^{2}}{p}\right)^{k_{j} / 2}\right),
$$

where $\widetilde{C}(k)$ is the $k$ th moment of a normal random variable with mean 0 and variance 1 , that is $\widetilde{C}(2 \ell)=(2 \ell) ! /\left(\ell ! 2^{\ell}\right)$ for $\ell$ a non-negative integer, and $\widetilde{C}(2 \ell+1)=0$.

Proof of Lemma 4.3. First, we extend $a(p)$ to a completely multiplicative function $a(n)$ by setting $a\left(p^{\alpha}\right)=a(p)^{\alpha}$. Also, let $p_{j}(n)$ equal one if $\Omega(n)=j$ and zero otherwise. We also define the multiplicative function $\nu\left(p^{\alpha}\right)=\alpha$ !. In this notation, we expand the moment as

$$
k_{1} ! \cdots k_{J} ! \sum_{\substack{r_{1}, \ldots, r_{J} \\ p_{j} \mid r_{j} \Rightarrow p_{j} \in I_{j}, j=1, \ldots, J}} \frac{a\left(r_{1}\right) \cdots a\left(r_{J}\right) p_{k_{1}}\left(r_{1}\right) \cdots p_{k_{J}}\left(r_{J}\right)}{\sqrt{r_{1} \cdots r_{J}} \nu\left(r_{1}\right) \cdots \nu\left(r_{J}\right)} \sum_{m}\left(\frac{m}{r_{1} \cdots r_{J}}\right) F\left(\frac{m}{X}\right) .
$$

Since $r_{1} \cdots r_{J} \leq x^{k_{1}+\cdots+k_{J}} \leq X^{\varepsilon}$ we can apply Proposition 3.1 (see Remark 1) to see that the inner sum over $m$ equals 0 unless $r_{1} \cdots r_{J}=\square$, and this implies that $r_{j}=\square$ for each $j=1, \ldots, J$, since $\left(r_{i}, r_{j}\right)=1$ for $i \neq j$. Since $\Omega\left(r_{j}\right)=k_{j}$ this completes the proof in the case where $k_{j}$ is odd for some $j$. 
To handle the remaining case write $r_{j}=n_{j}^{2}$ and $k_{j}=2 h_{j}$ so by Proposition 3.1 the above equation equals

$$
X \widehat{F}(0)\left(2 h_{1}\right) ! \cdots\left(2 h_{J}\right) ! \sum_{\substack{n_{1}, \ldots, n_{J} \\ p_{j} \mid n_{j} \Rightarrow p_{j} \in I_{j}, j=1, \ldots, J}} \frac{a\left(n_{1}\right)^{2} \cdots a\left(n_{J}\right)^{2} p_{h_{1}}\left(n_{1}\right) \cdots p_{h_{J}}\left(n_{J}\right)}{n_{1} \cdots n_{J} \nu\left(n_{1}^{2}\right) \cdots \nu\left(n_{J}^{2}\right)} \frac{\varphi\left(n_{1}^{2} \cdot n_{J}^{2}\right)}{n_{1}^{2} \cdots n_{J}^{2}} .
$$

Now apply the inequalities $\nu\left(n^{2}\right) \geq 2^{\Omega(n)} \nu(n)$ and $\varphi(n) / n \leq 1$ then rearrange the sum to see that the above is

$$
\begin{aligned}
& \leq X \widehat{F}(0)\left(2 h_{1}\right) ! \cdots\left(2 h_{J}\right) ! \sum_{\substack{n_{1}, \ldots, n_{J} \\
p_{j} \mid n_{j} \Rightarrow p_{j} \in I_{j}, j=1, \ldots, J}} \frac{a\left(n_{1}\right)^{2} \cdots a\left(n_{J}\right)^{2} p_{h_{1}}\left(n_{1}\right) \cdots p_{h_{J}}\left(n_{J}\right)}{n_{1} \cdots n_{J} 2^{\Omega\left(n_{1}\right)+\cdots+\Omega\left(n_{J}\right)} \nu\left(n_{1}\right) \cdots \nu\left(n_{J}\right)} \\
& =X \widehat{F}(0) \prod_{j \leq J} \frac{\left(2 h_{j}\right)}{2^{h_{j}} h_{j} !}\left(\sum_{p \in I_{j}} \frac{a(p)^{2}}{p}\right)^{h_{j}} .
\end{aligned}
$$

We now prove Lemma 4.2.

Proof of Lemma 4.2. We can assume at the outset that $(a, b) \mid \ell$ otherwise the result is vacuously true. In the notation of the proof of the previous lemma we have

$$
\begin{aligned}
& \sum_{\substack{m, n \\
a m=b n+\ell}}\left(\sum_{\substack{2<p \leq x \\
p \nmid a b}} \frac{a(p)\left(\frac{m}{p}\right)}{\sqrt{p}}\right)^{k}\left(\sum_{\substack{2<p \leq x \\
p \nmid a b}} \frac{a(p)\left(\frac{n}{p}\right)}{\sqrt{p}}\right)^{j} F\left(\frac{a m}{X}\right) \\
= & k ! j ! \sum_{\substack{r, s \\
p \mid r s \Rightarrow 2<\leq \leq x \\
(r s, a b)=1}} \frac{a(r) a(s) p_{k}(r) p_{j}(s)}{\sqrt{r s} \nu(r) \nu(s)} \sum_{\substack{m, n \\
a m=b n+\ell}}\left(\frac{m}{r}\right)\left(\frac{n}{s}\right) F\left(\frac{a m}{X}\right) .
\end{aligned}
$$

Let $\rho(n)$ be the multiplicative function given by $\rho\left(p^{\alpha}\right)=\left(p^{\alpha}, \ell\right) / p^{\alpha}$ and $\varsigma(n)$ be the multiplicative function given by $\varsigma\left(p^{\alpha}\right)=(1+3 / p)^{\alpha}$. Notice that the main term $\mathcal{M}\left(r_{0}, q, w, a, b, \ell\right)$ in Proposition 3.1 is bounded by

$$
\left|\sum_{\substack{d_{1}\left|q, d_{2}\right| w \\\left(d_{1} d_{2}, r_{0}\right)=1 \\\left(a d_{1}, b d_{2}\right) \mid \ell}} \frac{\mu\left(d_{1}\right) \mu\left(d_{2}\right)}{\left[d_{1}, d_{2}\right]} \frac{c_{r_{0}}\left(\ell /\left(a d_{1}, b d_{2}\right)\right)}{r_{0}}\right| \leq \rho\left(r_{0}\right) \varsigma(q w) .
$$


Write $r=r_{0} q^{2}$ and $s=s_{0} w^{2}$ where $r_{0}$ and $s_{0}$ are square-free and apply Proposition 3.1 to see that only the terms with $r_{0}=s_{0}=t$ survive and (4.1) is

$$
\ll \widehat{F}(0) \cdot \frac{X}{[a, b]} k ! j ! \sum_{\substack{t, q, w \\ p \mid t q w \Rightarrow p \leq x}} \frac{a^{2}(t q w) \rho(t) \varsigma(q w) p_{k}\left(t q^{2}\right) p_{j}\left(t w^{2}\right) \mu^{2}(t)}{t q w \nu\left(t n_{1}^{2}\right) \nu\left(t m_{1}^{2}\right)}
$$

Now notice that if $k$ and $j$ are of different parities, then $p_{k}\left(t q^{2}\right) p_{j}\left(t w^{2}\right)=0$. Set $\eta=1$ if $k$ and $j$ are both odd and set $\eta=0$ if $k$ and $j$ are both even. Without loss of generality assume $k \leq j$ and write $k_{0}=(k-\eta) / 2$ and $j_{0}=(j-\eta) / 2$. Thus, using the inequalities $1 \leq \nu(m) \nu(n) \leq \nu(m n)$ and $\nu\left(n^{2}\right) \geq 2^{\Omega(n)} \nu(n)$ we bound the above sum by

$$
\leq \widehat{F}(0) \cdot \frac{X}{[a, b]} k ! j ! \sum_{h=0}^{k_{0}} \sum_{\substack{t, q, w \\ p \mid t q w \Rightarrow p \leq x}} \frac{a^{2}(t q w) \rho(t) \varsigma(q) \varsigma(w) p_{2 h+\eta}(t) p_{k_{0}-h}(q) p_{j_{0}-h}(w)}{t q w 2^{\Omega(q)+\Omega(w)} \nu(t) \nu(q) \nu(w)}
$$

Let

$$
A(x)=\sum_{p \leq x} \frac{a(p)^{2} \rho(p)}{p} \quad \text { and } \quad B(x)=\frac{1}{2} \sum_{p \leq x} \frac{a(p)^{2}}{p} .
$$

Note that $A(x) \ll A(\ell)$. Rearranging (4.2) we see that it equals

$$
=\widehat{F}(0) \cdot \frac{X}{[a, b]} k ! j ! \sum_{h=0}^{k_{0}} \frac{A(x)^{2 h+\eta}(B(x)+O(1))^{k_{0}+j_{0}-2 h}}{\left(k_{0}-h\right) !\left(j_{0}-h\right) !(2 h+\eta) !} .
$$

To bound the above sum use the inequality $m^{n}(m-n) ! \geq m$ ! to get

$$
\begin{aligned}
\sum_{h=0}^{k_{0}} & \frac{A(x)^{2 h+\eta} \cdot(B(x)+O(1))^{k_{0}+j_{0}-2 h}}{\left(k_{0}-h\right) !\left(j_{0}-h\right) !(2 h+\eta) !} \\
& \ll \frac{A(x)^{\eta}(B(x)+O(1))^{k_{0}+j_{0}}}{k_{0} ! j_{0} !} \sum_{h=0}^{k_{0}} \frac{1}{(2 h+\eta) !} \cdot\left(\frac{\sqrt{k_{0} j_{0}} A(x)}{B(x)+O(1)}\right)^{2 h}
\end{aligned}
$$

Note that the inner sum over $h$ is

$$
\ll \exp \left(\frac{2 \sqrt{h_{0} k_{0}} A(x)}{B(x)}\right) \leq \exp \left(\frac{4 h_{0} A(x)}{B(x)}\right) \cdot \exp \left(\frac{4 k_{0} A(x)}{B(x)}\right) .
$$

Therefore (4.3) is

$$
\begin{aligned}
& \ll \widehat{F}(0) \cdot \frac{X}{[a, b]} \cdot \frac{k ! j !}{k_{0} ! j_{0} !} \cdot\left(e^{4 A(x) / B(x)} \cdot(B(x)+O(1))^{k_{0}+j_{0}}\right) \cdot A(x)^{\eta} \\
& \ll \widehat{F}(0) \cdot \frac{X}{[a, b]} \cdot \frac{k ! j !}{k_{0} ! j_{0} !} \cdot(B(x)+O(A(x)))^{k_{0}+j_{0}} \cdot A(x)^{\eta} .
\end{aligned}
$$


Since $B(x) \gg 1+A(x)$ by assumption we have $e^{4 A(x) / B(x)}=1+O(A(x) / B(x))$.

We are finally ready to prove the main result of this section.

Proof of Lemma 4.1. For fundamental discriminants $d_{1}, d_{2}$ we have,

$$
\chi_{d_{1}}(p)=\left(\frac{d_{1}}{p}\right) \text { and } \chi_{d_{2}}(p)=\left(\frac{d_{2}}{p}\right) .
$$

Thus after replacing $\chi_{d}$ by the corresponding Jacobi symbol, we extend the summation over $d_{1}, d_{2}$ to all integers, and not just fundamental discriminants. Also, let $F$ be a Schwartz function such that $\widehat{F}$ has compact support in $(-10,10)$ and $F(x) \geq \mathbf{1}_{[-1,1]}(x)$ for all $x \in \mathbb{R}$. Using the binomial theorem and the previous lemma we see that the $2 k$-th moment over fundamental discriminants is bounded by

$$
\begin{aligned}
\sum_{\substack{m, n \\
a m=b n+\ell}} & \left(\sum_{\substack{2<p \leq x \\
p \nmid a b}} \frac{a(p)\left(\left(\frac{m}{p}\right)+\left(\frac{n}{p}\right)\right)}{\sqrt{p}}\right)^{2 k} F\left(\frac{a m}{X}\right) \\
& =\sum_{j \leq 2 k}\left(\begin{array}{c}
2 k \\
j
\end{array}\right) \sum_{\substack{m, n \\
a m=b n+\ell}}\left(\sum_{\substack{2<p \leq x \\
p \nmid a b}} \frac{a(p)\left(\frac{m}{p}\right)}{\sqrt{p}}\right)^{j}\left(\sum_{\substack{2<p<x \\
p \nmid a b}} \frac{a(p)\left(\frac{n}{p}\right)}{\sqrt{p}}\right)^{2 k-j} F\left(\frac{a m}{X}\right) .
\end{aligned}
$$

Writing

$$
B(x)=\frac{1}{2} \sum_{p \leq x} \frac{a(p)^{2}}{p} \text { and } A(\ell)=1+\sum_{p \mid \ell} \frac{a(p)^{2}}{p}
$$

and applying the previous lemma we bound this as

$$
\ll \frac{X}{[a, b]}(2 B(x)+O(A(\ell)))^{k} \sum_{j \leq 2 k}\left(\begin{array}{c}
2 k \\
j
\end{array}\right) C(2 k-j) C(j)\left(\frac{A(\ell)}{B(x)}\right)^{\eta(2 k-j, j)},
$$

where $\eta(k, j)$ equals one if $k$ and $j$ have the same parity and is zero otherwise. The contribution of the even $j$ is bounded by

$$
\ll \frac{(2 k) !}{2^{k}} \sum_{j=0}^{k} \frac{1}{(k-j) ! j !}=\frac{(2 k) !}{k !} .
$$

Whereas the odd $j$ in (4.4) contribute

$$
\ll \frac{A(\ell)}{B(x)} \frac{(2 k) !}{2^{k-1}} \sum_{j=0}^{k-1} \frac{1}{(k-1-j) ! j !}=\frac{k A(\ell)}{B(x)} \cdot \frac{(2 k) !}{k !} \leq \frac{(2 k) !}{k !}\left(1+\frac{A(\ell)}{B(x)}\right)^{k},
$$

where in the last inequality we used the elementary estimate $k w<(1+w)^{k}$ for $k \geq 1, w>0$. Using the above two estimates in (4.4) completes the proof. 


\section{LEMMAS ON L-FUNCTIONS}

Let $f$ be an even, arithmetically normalized, weight 0 Hecke Maaß cusp form for $\mathrm{SL}_{2}(\mathbb{Z})$ with Laplace eigenvalue $-\left(\frac{1}{4}+t^{2}\right)$. We will need rather sharp estimates for the following two moments,

$$
\sum_{|d| \leq X} L\left(\frac{1}{2}, f \otimes \chi_{d}\right) \quad \text { and } \quad \sum_{\begin{array}{r}
d_{1}, d_{2} \\
a\left|d_{1}\right| \leq X \\
a d_{1}=b d_{2}+\ell
\end{array}} L\left(\frac{1}{2}, f \otimes \chi_{d_{1}}\right)^{1 / 2} L\left(\frac{1}{2}, f \otimes \chi_{d_{2}}\right)^{1 / 2}
$$

where the summation is over fundamental discriminants. Before stating our lemmas let us explain heuristically what to expect.

5.1. Heuristics. Heuristically for $|d| \gg|t|^{\varepsilon}$ we expect $\log L\left(\frac{1}{2}, f \otimes \chi_{d}\right)$ to be approximated by

$$
\sum_{p^{k} \leq|d|^{\varepsilon}} \frac{\left(\alpha_{p}^{k}+\beta_{p}^{k}\right) \chi_{d}(p)^{k}}{k p^{k / 2}}
$$

where $\alpha_{p}, \beta_{p}$ are the Satake parameters. We also expect that as $d$ varies over fundamental discriminants $\chi_{d}(p)$ behaves on average as an independent random variable $X(p)$ taking values \pm 1 with equal probability $\frac{1}{2(p+1)}$ and the value 0 with probability $\frac{1}{p+1}$. We note that in the sum the terms with $k \geq 3$ contribute $O(1)$, while for $k=2$ we have $\alpha_{p}^{2}+\beta_{p}^{2}=\lambda_{f}\left(p^{2}\right)-1$. Therefore the above sum heuristically behaves like

$$
\sum_{p \leq|d|^{\varepsilon}} \frac{\lambda_{f}(p) X(p)}{\sqrt{p}}+\frac{1}{2} \sum_{p \leq|d|^{\varepsilon / 2}} \frac{\lambda_{f}\left(p^{2}\right)}{p}-\frac{1}{2} \log \log |d|+O(1)
$$

which is the same as

$$
\sum_{p \leq|d|^{\varepsilon}} \frac{\lambda_{f}(p) X(p)}{\sqrt{p}}+\frac{1}{2} \log L\left(1, \operatorname{Sym}^{2} f\right)-\frac{1}{2} \log \log |d|+O(1) .
$$

In addition the sum over $p \leq|d|^{\varepsilon}$ behaves approximately like a Gaussian random variable with mean 0 and variance $\sum_{p \leq|d|^{\varepsilon}} \lambda_{f}(p)^{2} / p=\log \log |d|+\log L\left(1, \operatorname{Sym}^{2} f\right)+$ $O(1)$, since $\lambda_{f}(p)^{2}=\lambda_{f}\left(p^{2}\right)+1$. Note that we have to include $L\left(1, \operatorname{Sym}^{2} f\right)$ since a priori its contribution could easily overwhelm $\log \log |d|$ when $|t|^{\varepsilon} \leq|d| \leq t^{2}$ (this is due to our lack of knowledge of the Ramanujan-Petersson conjecture in the case of weight 0 Maaß forms). It follows from these considerations that we expect heuristically, in the range $|t|^{\varepsilon} \leq X$, that

$$
\begin{aligned}
\sum_{X \leq|d| \leq 2 X} L\left(\frac{1}{2}, f \otimes \chi_{d}\right) & \asymp X \cdot \mathbb{E}\left[\exp \left(\sum_{2<p \leq X} \frac{\lambda_{f}(p) X(p)}{\sqrt{p}}\right)\right](\log X)^{-1 / 2} \cdot L\left(1, \operatorname{Sym}^{2} f\right)^{1 / 2} \\
& \asymp L\left(1, \operatorname{Sym}^{2} f\right) X
\end{aligned}
$$


using the fact that $\mathbb{E}\left[e^{\lambda X}\right]=\exp \left(\frac{1}{2} \sigma^{2} \lambda^{2}\right)$ for a Gaussian random variable with variance $\sigma^{2}$ and mean 0 . In the shifted moment the same heuristic applies, but in addition we expect $L\left(\frac{1}{2}, f \otimes \chi_{d_{1}}\right)$ to behave approximately independently from $L\left(\frac{1}{2}, f \otimes \chi_{d_{2}}\right)$. This leads to the following conjecture, for $1 \leq a, b,|\ell|<X^{1-\varepsilon}$

$$
\begin{aligned}
\sum_{\substack{d_{1}, d_{2} \\
X \leq a\left|d_{1}\right| \leq 2 X \\
a d_{1}=b d_{2}+\ell}} L\left(\frac{1}{2}, f \otimes \chi_{d_{1}}\right)^{1 / 2} L\left(\frac{1}{2}, f \otimes \chi_{d_{2}}\right)^{1 / 2} \\
\asymp \frac{X}{[a, b]}\left[\mathbb{E} \exp \left(\frac{1}{2} \sum_{p \leq X} \frac{\lambda_{f}(p) X(p)}{\sqrt{p}}\right)\right]^{2} \cdot(\log X)^{-1 / 2} L\left(1, \operatorname{Sym}^{2} f\right)^{1 / 2} \\
\asymp \frac{X}{[a, b]} \cdot L\left(1, \mathrm{Sym}^{2} f\right)^{3 / 4} \cdot(\log X)^{-1 / 4} .
\end{aligned}
$$

5.2. Rigorous results under GRH. We establish the following two lemmas which combine Soundararajan's method for moments of $L$-functions along with our character sum estimate.

Lemma 5.1. Assume the Generalized Riemann Hypothesis. Let $f$ be an even, arithmetically normalized, Hecke-Maaß eigencuspform with eigenvalue $-\left(\frac{1}{4}+t^{2}\right)$. Then, uniformly for $|t|^{\varepsilon}<X, 0 \neq|\ell| \leq \log X$, and $1 \leq a, b \leq(\log X)^{100}$,

$$
\sum_{\substack{d_{1}, d_{2} \\ a\left|d_{1}\right| \leq X \\ a d_{1}=b d_{2}+\ell}} L\left(\frac{1}{2}, f \otimes \chi_{d_{1}}\right)^{1 / 2} L\left(\frac{1}{2}, f \otimes \chi_{d_{2}}\right)^{1 / 2} \ll_{\varepsilon} L\left(1, \operatorname{Sym}^{2} f\right)^{3 / 4+\varepsilon} \frac{X}{[a, b]}(\log X)^{-1 / 4+\varepsilon},
$$

where the summation is over fundamental discriminants $d_{1}, d_{2}$.

When using this lemma in combination with a Waldspurger type formula we will be dividing by $L\left(1, \operatorname{Sym}^{2} f\right)$. We therefore record the following simple (but useful!) observation.

Lemma 5.2. Assume the Generalized Riemann Hypothesis. Let $f$ be an even, arithmetically normalized, Hecke-Maaß eigencuspform with eigenvalue $-\left(\frac{1}{4}+t^{2}\right)$. Then,

$$
L\left(1, \operatorname{Sym}^{2} f\right) \gg \frac{1}{\log \log |t|} .
$$

Proof. By Perron's formula, for $x \geq 1$

$$
\frac{1}{2 \pi i} \int_{1-i x \log |t|}^{1+i x \log |t|} \log \left(L\left(s+1, \operatorname{Sym}^{2} f\right)\right) \frac{x^{s}}{s} d s=\sum_{p \leq x} \frac{\lambda_{f}\left(p^{2}\right)}{p}+O(1) .
$$


Under GRH, $\log \left(L\left(s+1, \operatorname{Sym}^{2} f\right)\right)$ is analytic in the half-plane $\operatorname{Re}(s)>-\frac{1}{2}$. Hence shifting contours to $\operatorname{Re}(s)=-\frac{1}{2}+\frac{1}{\log x}$, collecting a simple at $s=0$ with residue equal to $\log L\left(1, \operatorname{Sym}^{2} f\right)$, and bounding the horizontal contours by $O(1)$ we see that

$$
\sum_{p \leq x} \frac{\lambda_{f}\left(p^{2}\right)}{p}=\log L\left(1, \operatorname{Sym}^{2} f\right)+O\left(1+x^{-1 / 2} \int_{-x \log |t|}^{x \log |t|} \frac{\log (|t|+|\tau|)}{1+\tau} d \tau\right) .
$$

Since $\lambda_{f}\left(p^{2}\right)=\lambda_{f}(p)^{2}-1 \geq-1$ it follows that the LHS is at least $-\log \log x+O(1)$. Choosing $x=(\log |t|)^{2+\varepsilon}$ the claim follows.

We also need an upper bound for the first moment, however Soundararajan's method only gives

$$
\sum_{|d| \leq X} L\left(\frac{1}{2}, f \otimes \chi_{d}\right) \ll L\left(1, \operatorname{Sym}^{2} f\right)^{1+\varepsilon} \cdot X(\log X)^{\varepsilon}
$$

where the summation is over fundamental discriminants. This bound differs from the one predicted in the heuristic of the previous section by an extra factor of $L\left(1, \operatorname{Sym}^{2} f\right)^{\varepsilon}(\log X)^{\varepsilon}$ and the term $L\left(1, \operatorname{Sym}^{2} f\right)^{\varepsilon}$ is especially problematic in our range of interest $|t|^{\varepsilon} \leq X \leq t^{2}$ since even on GRH we only know that

$$
L\left(1, \operatorname{Sym}^{2} f\right) \ll \exp \left((\log |t|)^{1 / 8+\varepsilon}\right)
$$

which is proved by Li [23]. However the loss of $\varepsilon$ on the exponent $L\left(1, \operatorname{Sym}^{2} f\right)$ is due to a non-optimal treatment of the small primes $p \ll(\log |t|)^{2+\varepsilon}$ in Soundararajan's method [47]. We are in luck since small primes are analytically easy to deal with, and we remedy this loss by a small refinement of Soundararajan's method which is more efficient on small primes. We also note that in principle the loss of $(\log X)^{\varepsilon}$ could have been also averted by the use of Harper's [9] refinement of Soundararajan's method.

Lemma 5.3. Assume the Generalized Riemann Hypothesis. Let $f$ be an even, arithmetically normalized Hecke-Maaß eigencuspform with eigenvalue $-\left(\frac{1}{4}+t^{2}\right)$. Then, uniformly in $|t|^{\varepsilon}<X$,

$$
\sum_{|d| \leq X} L\left(\frac{1}{2}, f \otimes \chi_{d}\right) \ll L\left(1, \operatorname{Sym}^{2} f\right) X(\log X)^{\varepsilon}
$$

where the summation is over fundamental discriminants.

For the proof of both Lemma 5.3 and Lemma 5.1 we will use a theorem of Chandee [3] which is a generalization of an inequality first obtained by Soundararajan for the Riemann zeta-function in [47]. 
Lemma 5.4. Assume the Generalized Riemann Hypothesis. Let $f$ be an even, arithmetically normalized Hecke-Maaß eigencuspform with eigenvalue $-\left(\frac{1}{4}+t^{2}\right)$ for the full modular group. Let d be a fundamental discriminant. Then,

$$
\log L\left(\frac{1}{2}, f \otimes \chi_{d}\right) \leq \sum_{p^{n} \leq x} \frac{\chi_{d}\left(p^{n}\right)\left(\alpha_{p}^{n}+\beta_{p}^{n}\right)}{n p^{n(1 / 2+1 / \log x)}} \cdot \frac{\log \left(x / p^{n}\right)}{\log x}+O\left(\frac{\log (|d|+|t|)}{\log x}+1\right)
$$

where $\alpha_{p}, \beta_{p}$ are the Satake parameters.

Proof. See Theorem 2.1 of $[3]$.

Remark 2. Note that $\alpha_{p}^{n}+\beta_{p}^{n} \in \mathbb{R}$ for all $p$ and $n \geq 1$, regardless of the truth of the Ramanujan-Petersson conjecture. Since $\lambda_{f}(p)=\alpha_{p}+\beta_{p}$ and $\alpha_{p} \beta_{p}=1$, it follows that $\alpha_{p}$ and $\beta_{p}$ are roots of $x^{2}-\lambda_{f}(p) x+1$. If $\left|\lambda_{f}(p)\right|>2$ then the discriminant of this polynomial is positive, hence its roots $\alpha_{p}, \beta_{p}$ are real, and therefore $\alpha_{p}^{n}+\beta_{p}^{n}$ is real. On the other hand if $\left|\lambda_{f}(p)\right| \leq 2$ then the roots are complex, lie on the unit circle and are conjugates of each other, and hence $\alpha_{p}^{n}+\beta_{p}^{n}=2 \cos \left(n \theta_{p}\right)$ for some $\theta_{p} \in \mathbb{R}$.

Additionally the proof of Lemma 5.3 and Lemma 5.1 will require the following very weak estimate for the second moment. This relies on the following lemma.

Lemma 5.5. Assume the Generalized Riemann Hypothesis. Let $f$ be an even, arithmetically normalized Hecke-Maaß eigencuspform with eigenvalue $\left(-\frac{1}{4}+t^{2}\right)$. Then, uniformly in $|t|^{\varepsilon} \leq X$, and $x \leq X$,

$$
\sum_{p \leq x} \frac{\lambda_{f}(p)^{2}}{p} \ll(\log X)^{1 / 3}
$$

Proof. If $x \leq(\log |t|)^{32 / 23}$ then we apply the Kim-Sarnak bound $\left|\lambda_{f}(p)\right| \leq p^{7 / 64}$, to see that,

$$
\sum_{p \leq x} \frac{\lambda_{f}(p)^{2}}{p} \ll x^{7 / 32+\varepsilon} \ll(\log |t|)^{7 / 23+\varepsilon} \ll(\log X)^{1 / 3} .
$$

If $x>(\log |t|)^{32 / 23}$ then, by (5.1) and using Li's bound (5.2), we see that,

$$
\begin{aligned}
\sum_{p \leq x} \frac{\lambda_{f}(p)^{2}}{p}=\sum_{p \leq x} \frac{1+\lambda_{f}\left(p^{2}\right)}{p} & \ll \log \log x+(\log |t|)^{1 / 8+\varepsilon}+x^{-1 / 2+\varepsilon}(\log |t|)^{1+\varepsilon} \\
& \ll \log \log x+(\log |t|)^{7 / 23+\varepsilon} \ll(\log X)^{1 / 3}
\end{aligned}
$$

for $x \leq X$, as needed. 
Lemma 5.6. Assume the Generalized Riemann Hypothesis. Let $f$ be an even, arithmetically normalized Hecke-Maaß eigencusp form with eigenvalue $-\left(\frac{1}{4}+t^{2}\right)$. Then, uniformly in $|t|^{\varepsilon} \leq X$,

$$
\sum_{|d| \leq X} L\left(\frac{1}{2}, f \otimes \chi_{d}\right)^{2} \ll X \exp \left((\log X)^{1 / 3+\varepsilon}\right)
$$

where the summation is over fundamental discriminants.

Proof of Lemma 5.6. Let

$$
\mathcal{S}(X, \mathcal{V})=\left\{|d| \leq X: \log L\left(\frac{1}{2}, f \otimes \chi_{d}\right)>\mathcal{V}\right\} .
$$

Observe that

$$
\sum_{|d| \leq X} L\left(\frac{1}{2}, f \otimes \chi_{d}\right)=\int_{\mathbb{R}} e^{\mathcal{V}} \# \mathcal{S}(X, \mathcal{V}) d \mathcal{V}
$$

Thus, it will be enough to show that for $(\log X)^{1 / 3+\delta}<\mathcal{V}$, with $\delta>0$ arbitrarily small but fixed, we have,

$$
\# \mathcal{S}(X, \mathcal{V}) \ll X \exp (-c \mathcal{V} \log \mathcal{V})
$$

with the constant $c>0$ depending at most on $\delta$. Note that this bound is vacuous once $\mathcal{V}>A \log X / \log \log X$ for some large enough constant $A>0$, since choosing $x=(\log |d| t)^{\varepsilon}$ in Lemma 5.4 shows that $\log L\left(\frac{1}{2}, f \otimes \chi_{d}\right) \ll \log X / \log \log X$. To obtain (5.3) take $x=X^{1 /(\varepsilon \mathcal{V})}$. By Lemma 5.4.

$$
\log L\left(\frac{1}{2}, f \otimes \chi_{d}\right) \leq \sum_{p^{n} \leq x} \frac{\chi_{d}\left(p^{n}\right)\left(\alpha_{p}^{n}+\beta_{p}^{n}\right)}{n p^{n\left(\frac{1}{2}+1 / \log x\right)}} \cdot \frac{\log \left(x / p^{n}\right)}{\log x}+O\left(\frac{\log (|d|+|t|)}{\log x}+1\right) .
$$

In the sum the $p^{n}$ with $n \geq 3$ contribute $O(1)$. On the other hand the contribution from the $p^{2} \leq x$ is according to Lemma 5.5 less than,

$$
\sum_{2<p \leq \sqrt{x}} \frac{\lambda_{f}(p)^{2}+2}{p} \ll(\log X)^{1 / 3+\delta / 2}
$$

We conclude that if $\log L\left(\frac{1}{2}, f \otimes \chi_{d}\right)>\mathcal{V}$, then

$$
\frac{1}{2} \mathcal{V}<\sum_{p \leq x} \frac{\lambda_{f}(p) \chi_{d}(p)}{p^{\frac{1}{2}+1 / \log x}} \frac{\log x / p}{\log x} .
$$

Using Markov's inequality and Lemma 4.3 it follows that the number of fundamental discriminants $d$ for which this holds is

$$
\ll \frac{2^{2 k}}{\mathcal{V}^{2 k}} \sum_{|d| \leq X}\left(\sum_{p \leq x} \frac{\lambda_{f}(p) \chi_{d}(p)}{p^{\frac{1}{2}+1 / \log x}} \frac{\log x / p}{\log x}\right)^{2 k} \ll X \cdot\left(\frac{8 k}{e \mathcal{V}^{2}} \sum_{p \leq x} \frac{\lambda_{f}(p)^{2}}{p}\right)^{k} .
$$


By Lemma 5.5 the sum over primes on the RHS is $\ll(\log X)^{1 / 3}$. Picking $k=\lfloor\varepsilon \mathcal{V}\rfloor$, we see that since $\mathcal{V}>(\log X)^{1 / 3+\delta}$, there is an absolute constant $c>0$, such that the RHS of (5.4) is $\ll X e^{-c \delta \mathcal{V} \log \mathcal{V}}$ which establishes (5.3) , thus giving the lemma.

We are now ready to prove the main results of this section, namely Lemma 5.1 and Lemma 5.3 .

Proof of Lemma 5.1. Using the moment calculation we now deduce the lemma by the method of Soundararajan [47], with appropriate modifications. For fundamental discriminants $d_{1}, d_{2}$ write $\mathcal{L}_{f}\left(d_{1}, d_{2}\right)=L\left(\frac{1}{2}, f \otimes \chi_{d_{1}}\right) L\left(\frac{1}{2}, f \otimes \chi_{d_{2}}\right)$, and set

$$
\mathcal{S}(X, \mathcal{V})=\left\{d_{1}, d_{2}: a\left|d_{1}\right| \leq X, a d_{1}=b d_{2}+\ell, \log \mathcal{L}_{f}\left(d_{1}, d_{2}\right) \geq \mathcal{V}\right\}
$$

Let

$$
\begin{aligned}
\mu(f, X) & =-\log \log X+\log L\left(1, \operatorname{Sym}^{2} f\right), \\
\sigma^{2}(f, X) & =2 \log \log X+2 \log L\left(1, \operatorname{Sym}^{2} f\right) .
\end{aligned}
$$

Observe that for $\mathcal{U}=\exp (\sqrt{\log X})$,

$$
\begin{gathered}
\sum_{\begin{array}{c}
d_{1}, d_{2} \\
a\left|d_{1}\right| \leq X \\
a d_{1}=b d_{2}+\ell \\
\mathcal{L}_{f}\left(d_{1}, d_{2}\right)>\mathcal{U}
\end{array}}\left(\mathcal{L}_{f}\left(d_{1}, d_{2}\right)\right)^{1 / 2} \leq \mathcal{U}^{-1 / 2} \sum_{\begin{array}{c}
d_{1}, d_{2} \\
a\left|d_{1}\right| \leq X \\
a d_{1}=b d_{2}+\ell
\end{array}} \mathcal{L}_{f}\left(d_{1}, d_{2}\right) \\
\ll X \mathcal{U}^{-1 / 2} \cdot \exp \left((\log X)^{1 / 3+\delta}\right) \ll \frac{X}{\log X}
\end{gathered}
$$

by Lemma 5.6. On the other hand,

$$
\begin{aligned}
\sum_{\substack{d_{1}, d_{2} \\
a\left|d_{1}\right| \leq X \\
a d_{1}=b d_{2}+\ell \\
\mathcal{L}_{f}\left(d_{1}, d_{2}\right) \leq \mathcal{U}}}\left(\mathcal{L}_{f}\left(d_{1}, d_{2}\right)\right)^{1 / 2} & =\frac{1}{2} \cdot \int_{-\infty}^{\sqrt{\log X}} e^{\frac{1}{2} \cdot \mathcal{V}} \# \mathcal{S}(X, \mathcal{V}) d \mathcal{V} \\
& \leq \frac{L\left(1, \operatorname{Sym}^{2} f\right)^{1 / 2}}{2(\log X)^{1 / 2}} \int_{-\infty}^{2 \sqrt{\log X}} e^{\frac{1}{2} \cdot \mathcal{V}} \# \mathcal{S}(X, \mathcal{V}+\mu(f, X)) d \mathcal{V}
\end{aligned}
$$

The portion of the integral over $\mathcal{V} \leq \varepsilon \log \log X$ is bounded by

$$
\ll \frac{X}{[a, b]}(\log X)^{-1 / 2+\varepsilon} L\left(1, \operatorname{Sym}^{2} f\right)^{1 / 2},
$$

which is negligible since $L\left(1, \operatorname{Sym}^{2} f\right)^{1 / 2} \ll(\log X)^{\varepsilon} L\left(1, \operatorname{Sym}^{2} f\right)^{3 / 4}$ by Lemma 5.2 ,

Thus, our present lemma follows once we have shown that

$$
\# \mathcal{S}(X, \mathcal{V}+\mu(f, X)) \ll \frac{X}{[a, b]} \cdot \begin{cases}\exp \left(-\frac{\mathcal{V}^{2}}{2 \sigma^{2}(f, X)}(1+o(1))\right) & \text { if } \mathcal{V} \in I_{1}(X) \\ \exp \left(-c \mathcal{V} \log \frac{\mathcal{V}}{\sigma^{2}(f, X)}\right) & \text { if } \mathcal{V} \in I_{2}(X)\end{cases}
$$


where $c>0$ is an absolute constant, $I_{1}(X)=\left(\varepsilon \log \log X, \varepsilon \sigma^{2}(f, X) \log \sigma(f, X)\right]$ and $I_{2}(X)=\left(\varepsilon \sigma^{2}(f, X) \log \sigma(f, X), 2 \sqrt{\log X}\right]$.

It remains to establish (5.5). By Lemma 5.4,

$$
\begin{aligned}
\log \mathcal{L}_{f}\left(d_{1}, d_{2}\right) \leq \sum_{p^{n} \leq x} \frac{\left(\chi_{d_{1}}\left(p^{n}\right)+\chi_{d_{2}}\left(p^{n}\right)\right)\left(\alpha_{p}^{n}+\beta_{p}^{n}\right)}{n p^{n\left(\frac{1}{2}+1 / \log x\right)}} & \frac{\log \left(x / p^{n}\right)}{\log x} \\
& +O\left(\frac{\log \left(\left|d_{1} d_{2}\right|+|t|\right)}{\log x}+1\right) .
\end{aligned}
$$

In the sum over prime powers the terms with $n \geq 3$ contribute $O(1)$, which is negligible. Using the Hecke relations the sum over prime squares is for $x>(\log |t|)^{2+\varepsilon}$

$$
\begin{aligned}
\sum_{p \leq \sqrt{x}} \frac{\left(\chi_{d_{1}}\left(p^{2}\right)+\chi_{d_{2}}\left(p^{2}\right)\right)\left(\lambda_{f}^{2}(p)-2\right)}{2 p^{1+2 / \log x} \frac{\log x / p^{2}}{\log x}} & \leq \sum_{p \leq x} \frac{\lambda_{f}^{2}(p)}{p}-2 \log \log x+O\left(\sum_{p \mid d_{1} d_{2}} \frac{1}{p}\right) \\
& =\mu(f, x)+O\left(\log \log \log \left(\left|d_{1} d_{2}\right|+e^{e}\right)\right)
\end{aligned}
$$

by (5.1). Hence, for $x>(\log |t|)^{2+\varepsilon}$

$$
\begin{aligned}
\log \mathcal{L}_{f}\left(d_{1}, d_{2}\right)-\mu(f, x) \leq & \sum_{2<p \leq x} \frac{\left(\chi_{d_{1}}(p)+\chi_{d_{2}}(p)\right) \lambda_{f}(p)}{p^{\frac{1}{2}+1 / \log x}} \frac{\log x / p}{\log x} \\
& +O\left(\frac{\log \left(\left|d_{1} d_{2}\right|+|t|\right)}{\log x}+\log \log \log \left(\left|d_{1} d_{2}\right|+e^{e}\right)\right) .
\end{aligned}
$$

Also,

$$
\sum_{p \mid a b} \frac{\left|\lambda_{f}(p)\right|}{\sqrt{p}}<\omega(a b)=o(\log \log X) .
$$

since $a, b \leq(\log X)^{100}$. Combining the previous two observations and taking $x=$ $X^{1 /(\varepsilon \mathcal{V})}$ it follows that for all $X$ large enough,

$$
\log \mathcal{L}_{f}\left(d_{1}, d_{2}\right)-\mu(f, X) \leq \sum_{\substack{2<p \leq x \\ p \nmid a b}} \frac{\left(\chi_{d_{1}}(p)+\chi_{d_{2}}(p)\right) \lambda_{f}(p)}{p^{\frac{1}{2}+1 / \log x}} \frac{\log x / p}{\log x}+\frac{\varepsilon}{2} \mathcal{V}
$$

Let $z=x^{1 / \log \log X}$ and note that if the LHS of $(\underline{5.6})$ is at least $\mathcal{V}$ then

$$
\left|\sum_{\substack{2<p \leq z \\ p \nmid a b}} \frac{\left(\chi_{d_{1}}(p)+\chi_{d_{2}}(p)\right) \lambda_{f}(p)}{p^{\frac{1}{2}+1 / \log x}} \frac{\log x / p}{\log x}\right| \geq \mathcal{V}(1-\varepsilon)
$$


or

$$
\left|\sum_{\substack{z<p \leq x \\ p \nmid a b}} \frac{\left(\chi_{d_{1}}(p)+\chi_{d_{2}}(p)\right) \lambda_{f}(p)}{p^{\frac{1}{2}+1 / \log x}} \frac{\log x / p}{\log x}\right| \geq \frac{\varepsilon}{2} \mathcal{V} .
$$

Now we use Markov's inequality and apply Lemma 4.1 to see that for $0 \neq|\ell| \leq \log X$ the number of $d_{1}, d_{2}$ with $a\left|d_{1}\right| \leq X$ and $a d_{1}=b d_{2}+\ell$, for which (5.7) holds is bounded by, for any $k \leq \varepsilon \mathcal{V} \log \log X$,

$$
\begin{aligned}
& \frac{1}{(\mathcal{V}(1-\varepsilon))^{2 k}} \sum_{\substack{d_{1}, d_{2} \\
a \mid d_{1} \leq X \\
a d_{1}=b d_{2}+\ell}}\left(\sum_{\substack{2<p \leq z \\
p \nmid a b}} \frac{\left(\chi_{d_{1}}(p)+\chi_{d_{2}}(p)\right) \lambda_{f}(p)}{p^{\frac{1}{2}+1 / \log x}} \frac{\log x / p}{\log x}\right)^{2 k} \\
& \ll \frac{X(2 k) !}{[a, b]\left(2 \mathcal{V}^{2}\right)^{k} k !}\left(\sigma^{2}(f, X)(1+o(1))\right)^{k} \ll \frac{X}{[a, b]}\left(\frac{2 k \sigma^{2}(f, X)(1+o(1))}{e \mathcal{V}^{2}}\right)^{k} .
\end{aligned}
$$

Note that the use of Lemma 4.1 is justfied, since for $|\ell| \leq \log X$,

$$
\sum_{p \mid \ell} \lambda_{f}(p)^{2} / p \ll \sum_{p \mid \ell} p^{-2 \delta} \ll(\log \log X)^{1-2 \delta}
$$

using the bound $|a(p)| \ll p^{1 / 2-\delta}$ (where importantly we know that $\delta>0$ following for example the works of [25]) while $\sum_{p \leq x} \lambda_{f}(p)^{2} / p \gg \log \log x$ as a consequence of GRH using (5.1), since $x=X^{1 /(\varepsilon \mathcal{V})}$ and $\mathcal{V} \leq 2 \sqrt{\log X}$. Also, we have $\log \log x \gg \log \log X$.

If $\mathcal{V} \leq \varepsilon \sigma^{2}(f, X) \log \log X$ then we choose $k=\left\lfloor\mathcal{V}^{2} /\left(2 \sigma^{2}(f, X)\right)\right\rfloor$ in (5.9), and in the remaining case we choose $k=\lceil\mathcal{V} / 2\rceil$. This leads to the following bound,

$$
\ll \frac{X}{[a, b]} \begin{cases}\exp \left(\frac{-\mathcal{V}^{2}(1+o(1))}{2 \sigma^{2}(f, X)}\right) & \text { if } \mathcal{V} \leq \varepsilon \sigma^{2}(f, X) \log \log X, \\ \exp \left(-\left(\frac{1}{2}+o(1)\right) \mathcal{V} \log \frac{\mathcal{V}}{\sigma^{2}(f, X)}\right) & \text { if } \varepsilon \sigma^{2}(f, X) \log \log X<\mathcal{V} \leq 2 \sqrt{\log X},\end{cases}
$$

for the number of fundamental discriminants $d_{1}, d_{2}$ with $a\left|d_{1}\right| \leq X$, and $a d_{1}=b d_{2}+\ell$ for which (5.7) holds.

It remains to bound the number of fundamental discriminants $d_{1}, d_{2}$, with $a\left|d_{1}\right| \leq$ $X$, and $a d_{1}=b d_{2}+\ell$ for which (5.8) holds. Since $\mathcal{V} \leq 2 \sqrt{\log X}$ it follows that $z=X^{1 /(\varepsilon \mathcal{V} \log \log X)}>(\log |t|)^{2+\varepsilon}$. Hence, under GRH it follows from (5.1) that

$$
\sum_{z<p \leq x} \frac{\lambda_{f}^{2}(p)}{p}=\sum_{p \leq x} \frac{\lambda_{f}(p)^{2}}{p}-\sum_{p \leq z} \frac{\lambda_{f}(p)^{2}}{p}=\log \frac{\log x}{\log z}+O(1) .
$$

We will now apply Markov's inequality and Lemma4.1. In Lemma4.1 $\operatorname{set} a(p)=0$ for $p<z$ and $a(p)=\lambda_{f}(p)$ otherwise. This way the conditions of the lemma are trivially 
verified, since $\sum_{p \mid \ell} a(p)^{2} / p=0$ because $|\ell| \leq \log X$ according to our assumption. Thus applying Markov's inequality and Lemma 4.1 as in (5.10) it follows that, for $\varepsilon \log \log X<\mathcal{V}<2 \sqrt{\log X}$, the number of $d_{1}, d_{2}$ with $a\left|d_{1}\right| \leq X$ and $a d_{1}=b d_{2}+\ell$ for which (5.8) holds is

$$
\ll \frac{X}{[a, b]}\left(\frac{2 k}{e(\varepsilon \mathcal{V})^{2}} \log \log \log X(1+o(1))\right)^{k} \ll \frac{X}{[a, b]} \exp (-\varepsilon \mathcal{V} \log \mathcal{V})
$$

where we chose $k=\lceil\varepsilon \mathcal{V}\rceil$. Combining (5.10), (5.11) gives (5.5), thereby completing the proof.

Finally we are ready to prove Lemma 5.3.

Proof of Lemma 5.3. Given a parameter $x$, an integer $m$, and interval $I \subset[1, x]$, let

$$
\mathcal{P}_{I}(m, x)=\sum_{p \in I} \frac{\lambda_{f}(p)\left(\frac{m}{p}\right)}{p^{1 / 2+1 / \log x}} \cdot \frac{\log (x / p)}{\log x} .
$$

Let $F$ be a Schwartz function with $\widehat{F}$ compactly supported in $(-10,10)$. We assume that in addition $F$ satisfies $F(x) \geq \mathbf{1}_{[-1,1]}(x)$ for all $x \in \mathbb{R}$. First, by Lemma 5.6 for $\mathcal{U}=e^{\frac{1}{2}(\log X)^{5 / 6}}$ we have

$$
\sum_{\substack{|d| \leq X \\ L\left(\frac{1}{2}, f \otimes \chi_{d}\right)>\mathcal{U}}} L\left(\frac{1}{2}, f \otimes \chi_{d}\right) \ll \mathcal{U}^{-1} \sum_{|d| \leq X} L\left(\frac{1}{2}, f \otimes \chi_{d}\right)^{2} \ll X
$$

Secondly, let $I=\left(2,(\log X)^{5}\right]$ and suppose that $x>e^{(\log X)^{1 / 6}}$. By Lemma 4.3 the number of fundamental discriminants $|d| \leq X$ for which $\left|\mathcal{P}_{I}(d ; X)\right| \geq \frac{1}{2}(\log X)^{5 / 6}$ is for $k=\left\lfloor(\log X)^{1-\varepsilon}\right\rfloor$

$$
\begin{aligned}
\leq \frac{4^{k}}{(\log X)^{5 k / 3}} \sum_{m \in \mathbb{Z}}\left|\mathcal{P}_{I}(m ; X)\right|^{2 k} F\left(\frac{m}{X}\right) & \ll X \frac{(2 k) !}{k ! 2^{k}} \cdot\left(\frac{4}{(\log X)^{5 / 3}} \cdot \sum_{p \in I} \frac{\lambda_{f}(p)^{2}}{p}\right)^{k} \\
& \ll X\left(\frac{8 k(\log X)^{1 / 3}}{e(\log X)^{5 / 3}}\right)^{k} \ll X e^{-(\log X)^{1-\varepsilon}}
\end{aligned}
$$

where we used Lemma 5.5 in the second bound. It follows that,

$$
\sum_{\substack{|d| \leq X \\\left|\mathcal{P}_{I}(d, X)\right|>\frac{1}{2}(\log X)^{5 / 6}}} L\left(\frac{1}{2}, f \otimes \chi_{d}\right) \leq\left(\sum_{|d| \leq X} L\left(\frac{1}{2}, f \otimes \chi_{d}\right)^{2}\right)^{1 / 2} \cdot X^{1 / 2} \exp \left(-(\log X)^{1-\varepsilon}\right) \ll X
$$


on using Lemma 5.6. Combining this along with (5.12) we have that

$$
\sum_{|d| \leq X} L\left(\frac{1}{2}, f \otimes \chi_{d}\right)=\sum_{\substack{|d| \leq X \\ \log L\left(\frac{1}{2}, f \otimes \chi_{d}\right) \leq \frac{1}{2}(\log X)^{5 / 6} \\\left|\mathcal{P}_{I}(d, X)\right| \leq \frac{1}{2}(\log X)^{5 / 6}}} L\left(\frac{1}{2}, f \otimes \chi_{d}\right)+O(X) .
$$

Define $\mathcal{L}_{f}(d, X)=L\left(\frac{1}{2}, f \otimes \chi_{d}\right) e^{-\mathcal{P}_{I}(d, X)}$ and

$$
\widetilde{\mathcal{S}}(X, \mathcal{V})=\sum_{\substack{|d| \leq X \\ \mathcal{P}_{I}(d, X) \leq \frac{1}{2}(\log X)^{5 / 6} \\ \log \mathcal{L}_{f}(d, X)>\mathcal{V}}} e^{\mathcal{P}_{I}(d, X)} .
$$

Then

$$
\begin{aligned}
\sum_{\substack{|d| \leq X \\
\log L\left(\frac{1}{2}, f \otimes \chi_{d}\right) \leq \frac{1}{2}(\log X)^{5 / 6} \\
\left|\mathcal{P}_{I}(d, X)\right| \leq \frac{1}{2}(\log X)^{5 / 6}}} L\left(\frac{1}{2}, f \otimes \chi_{d}\right) \leq & \sum_{\substack{|d| \leq X \\
\log \mathcal{L}_{f}(d, X) \leq(\log X)^{5 / 6} \\
\left|\mathcal{P}_{I}(d, X)\right| \leq \frac{1}{2}(\log X)^{5 / 6}}} \mathcal{L}_{f}(d, X) \cdot e^{\mathcal{P}_{I}(d, X)} \\
= & \int_{-\infty}^{(\log X)^{5 / 6}} e^{\mathcal{V}} \cdot \widetilde{\mathcal{S}}(X, \mathcal{V}) d \mathcal{V} .
\end{aligned}
$$

In particular, it follows from this along with (5.14) that

$$
\sum_{|d| \leq X} L\left(\frac{1}{2}, f \otimes \chi_{d}\right) \leq \int_{-\infty}^{(\log X)^{5 / 6}} e^{\mathcal{V}} \cdot \widetilde{\mathcal{S}}(X, \mathcal{V}) d \mathcal{V}+O(X)
$$

Hence, it suffices to show that the above integral is $\ll L\left(1, \operatorname{Sym}^{2} f\right) X(\log X)^{\varepsilon}$. Letting as usual $\widetilde{\mu}(f, X)=-\frac{1}{2} \log \log X+\frac{1}{2} \log L\left(1\right.$, Sym $\left.^{2} f\right)$ it will suffice to show that

$\widetilde{\mathcal{S}}(X, \mathcal{V}+\widetilde{\mu}(f, X)) \ll$

$$
X(\log X)^{\varepsilon} \cdot L\left(1, \operatorname{Sym}^{2} f\right)^{1 / 2} \cdot \begin{cases}e^{-\frac{\mathcal{V}^{2}}{2 \log \log X}(1+o(1))} & \text { if } \varepsilon \log \log X \leq \mathcal{V} \leq \varepsilon(\log \log X)^{2} \\ e^{-(\varepsilon+o(1)) \mathcal{V} \log \mathcal{V}} & \text { if } \varepsilon(\log \log X)^{2} \leq \mathcal{V} \leq(\log X)^{5 / 6}\end{cases}
$$

Given $\varepsilon \log \log X \leq \mathcal{V} \leq 2(\log X)^{5 / 6}$ set $x=X^{1 /(\varepsilon \mathcal{V})}\left(>e^{(\log X)^{1 / 6}}\right)$ and $z=$ $x^{1 / \log \log X}$. Define also $J_{1}=\left((\log X)^{5}, z\right]$ and $J_{2}=(z, x]$. Using Lemma 5.4 we see that for a fundamental discriminant $d$, GRH implies

$$
\log \mathcal{L}_{f}(d, X)-\widetilde{\mu}(f, X) \leq \mathcal{P}_{J_{1}}(d, x)+\mathcal{P}_{J_{2}}(d, x)+\left(\mathcal{P}_{I}(d, x)-\mathcal{P}_{I}(d, X)\right)+\frac{\varepsilon}{2} \mathcal{V}
$$

If the LHS of the above inequality is $>\mathcal{V}$ then we must have at least one of the following events occur: $\left.\mathcal{P}_{J_{1}}(d, X)\right)>\mathcal{V}(1-\varepsilon), \mathcal{P}_{J_{2}}(d, x)>\frac{\varepsilon}{4} \mathcal{V}$ or $\mathcal{P}_{I}(d, x)-\mathcal{P}_{I}(d, X)>$ 
$\frac{\varepsilon}{4} \mathcal{V}$. Let $\mathcal{V}_{1}=(1-\varepsilon) \mathcal{V}$ and $\mathcal{V}_{2}=\mathcal{V}_{3}=\frac{\varepsilon}{4} \mathcal{V}$. Therefore, for any $k_{1}, k_{2}, k_{3} \geq 1$, $\widetilde{\mathcal{S}}(X, \mathcal{V}+\widetilde{\mu}(f, X))$ is bounded above by

$$
\begin{aligned}
& \sum_{\substack{m \\
\left|\mathcal{P}_{I}(m, X)\right| \leq \frac{1}{2}(\log X)^{5 / 6}}}\left(\left(\frac{\mathcal{P}_{J_{1}}(m, X)}{\mathcal{V}_{1}}\right)^{2 k_{1}}+\left(\frac{\mathcal{P}_{J_{2}}(m)}{\mathcal{V}_{2}}\right)^{2 k_{2}}\right) e^{\mathcal{P}_{I}(m, X)} F\left(\frac{m}{X}\right) \\
& +e^{\frac{1}{2}(\log X)^{5 / 6}} \sum_{m}\left(\frac{\mathcal{P}_{I}(d, x)-\mathcal{P}_{I}(d, X)}{\mathcal{V}_{3}}\right)^{2 k_{3}} F\left(\frac{m}{X}\right) .
\end{aligned}
$$

To estimate (5.18) ) we note that $\left|p^{-1 / \log x} \frac{\log p / x}{\log x}-p^{-1 / \log X} \frac{\log p / X}{\log X}\right| \leq C \mathcal{V}(\log X)^{-1+\varepsilon}$ for $p \leq(\log X)^{5}$, and $C>1$ is a sufficiently large absolute constant. So applying Lemmas 4.3 and 5.5 we get for $k_{3}=\left\lfloor(\log X)^{1-\varepsilon}\right\rfloor$ that (5.18) is

$$
\begin{aligned}
& \ll X \frac{e^{\frac{1}{2}(\log X)^{5 / 6}}}{\mathcal{V}_{3}^{2 k_{3}}} \frac{\left(2 k_{3}\right) !}{2^{k_{3}} k_{3} !}\left(\frac{C^{2} \mathcal{V}^{2}}{(\log X)^{2-\varepsilon}} \sum_{p \in I} \frac{\lambda_{f}(p)^{2}}{p}\right)^{k_{3}} \\
& \ll X e^{\frac{1}{2}(\log X)^{5 / 6}}\left(\frac{32 C^{2}}{\varepsilon^{2} e(\log X)^{2 / 3-\varepsilon}}\right)^{(1+o(1))(\log X)^{1-\varepsilon}} \ll X e^{-(1+o(1))(\log X)^{1-\varepsilon}} .
\end{aligned}
$$

In order to bound (5.17) first observe that for $x \leq N / e^{2}$, where $N$ is an even integer

$$
e^{x} \leq 2 \sum_{h=0}^{N} \frac{x^{h}}{h !}
$$

Thus setting $M=\frac{1}{2}(\log X)^{5 / 6}$, we have that (5.17) is

$$
\leq 2 \sum_{i=1}^{2} \sum_{h \leq 2\left\lfloor e^{2} M\right\rfloor} \frac{1}{h !} \frac{1}{\mathcal{V}_{i}^{2 k_{i}}} \sum_{\substack{m \\\left|\mathcal{P}_{I}(m, X)\right| \leq M}} \mathcal{P}_{I}(m, X)^{h} \cdot \mathcal{P}_{J_{i}}(m, x)^{2 k_{i}} F\left(\frac{m}{X}\right) .
$$

We will now extend the inner sum over $m$ to all integers. To do this, we observe that for $k_{1}, k_{2} \leq \varepsilon \mathcal{V}<M$ the contribution of an identical sum over terms $m$ with $\left|\mathcal{P}_{I}(m, x)\right|>M$ is by Cauchy-Schwarz, Lemmas 4.3 and 5.5, and (5.13) seen to be

$$
\begin{aligned}
& \ll X e^{-(\log X)^{1-\varepsilon}} \cdot \sum_{i=1,2} \sum_{h \leq 2\left\lfloor e^{2} M\right\rfloor} h^{h / 2}\left(\sum_{p \in I} \frac{\lambda_{f}(p)^{2}}{p}\right)^{h / 2}\left(2 k_{i}\right)^{k_{i}}\left(\sum_{p \in J_{i}} \frac{\lambda_{f}(p)^{2}}{p}\right)^{k_{i}} \\
& \ll X e^{-(\log X)^{1-\varepsilon}} e^{M(\log M)^{2}} \ll X e^{-(\log X)^{1-\varepsilon}} .
\end{aligned}
$$

Hence, we can extend the inner sum in (5.20) to all of $m \in \mathbb{Z}$ at the cost of an error of size $X e^{-(\log X)^{1-\varepsilon}}$. Using Lemma 4.3 we evaluate the moments in the sum over all 
$m$ provided that $k_{1} \leq \varepsilon \mathcal{V} \log \log X$ and $k_{2} \leq \varepsilon \mathcal{V}$. Extending the summation in the resulting main terms to all integers $h \geq 0$ we get the following bound for (5.20),

$$
\begin{aligned}
& 2 \sum_{i=1,2} \sum_{h \leq 2\left\lfloor e^{2} M\right\rfloor} \frac{1}{h !} \frac{1}{\mathcal{V}_{i}^{2 k_{i}}} \sum_{m} \mathcal{P}_{I}(m, X)^{h} \mathcal{P}_{J_{i}}(m, x)^{2 k_{i}} F\left(\frac{m}{X}\right)+O\left(X e^{-(\log X)^{1-\varepsilon}}\right) \\
& \ll X \sum_{i=1,2}\left(\frac{2 k_{i}}{e \mathcal{V}_{i}^{2}}\right)^{k_{i}}\left(\sum_{p \in J_{i}} \frac{\lambda_{f}(p)^{2}}{p}\right)^{k_{i}} \sum_{h=0}^{\infty} \frac{1}{(2 h) !} \cdot \frac{(2 h) !}{2^{h} h !}\left(\sum_{p \in I} \frac{\lambda_{f}(p)^{2}}{p}\right)^{h}+X e^{-(\log X)^{1-\varepsilon}} .
\end{aligned}
$$

The sum over $I$ equals $\log L\left(1, \operatorname{Sym}^{2} f\right)+O(\log \log \log X)$ so that the sum over $h$ is $\ll L\left(1, \operatorname{Sym}^{2} f\right)^{1 / 2}(\log X)^{\varepsilon}$. We now specialize our choice of $k_{1}$ and $k_{2}$. Using the Hecke relations and applying (5.1)

$$
\sum_{p \in J_{1}} \frac{\lambda_{f}(p)^{2}}{p} \leq \log \log X+O(1), \quad \sum_{p \in J_{2}} \frac{\lambda_{f}(p)^{2}}{p}=\log \log \log X+O(1) .
$$

First for $k_{1}$, if $\varepsilon \log \log X \leq \mathcal{V} \leq \varepsilon(\log \log X)^{2}$ then choose $k_{1}=\left\lfloor\mathcal{V}^{2} /(2 \log \log X)\right\rfloor$. While if $\mathcal{V} \geq \varepsilon(\log \log X)^{2}$ then choose $k_{1}=\lfloor\varepsilon \mathcal{V} / 2\rfloor$. Secondly, for $k_{2}$ set $k_{2}=\lfloor\varepsilon \mathcal{V}\rfloor$. This along with our earlier estimate for the sum over $h$ shows that for this choice of $k_{1}$ and $k_{2}$, the RHS of (5.21) (and thus (5.17) as well) is bounded by

$$
\ll \begin{cases}L\left(1, \operatorname{Sym}^{2} f\right)^{1 / 2} X(\log X)^{\varepsilon} e^{-\frac{\mathcal{V}^{2}}{2 \log \log X}(1-o(1))} & \text { if } \varepsilon \log \log X<\mathcal{V}<\varepsilon(\log \log X)^{2}, \\ L\left(1, \operatorname{Sym}^{2} f\right)^{1 / 2} X(\log X)^{\varepsilon} e^{-(\varepsilon+o(1)) \mathcal{V} \log \mathcal{V}} & \text { if } \varepsilon(\log \log X)^{2} \leq \mathcal{V} \leq(\log X)^{5 / 6}\end{cases}
$$

Therefore, using this bound for (5.17) along with the estimate (5.19) for (5.18) gives (5.16), which completes the proof.

\section{Averages of COEFficients of HAlF-Integral Weight MAASS Forms}

Let $g_{j}$ be a basis of $V^{+}$consisting of simultaneous eigenfunctions of $\Delta_{1 / 2}$ and of the Hecke operators $T_{p^{2}}, p \neq 2$, and with each $g_{j}$ normalized so that $\iint_{\Gamma_{0}(4) \backslash \mathbb{H}}\left|g_{j}(z)\right|^{2} \operatorname{dvol}(z)=$ 1. Write $g=g_{j}$ with $\Delta_{1 / 2} g=-\left(\frac{1}{4}+t^{2}\right) g$. As usual $g(z)$ has a Fourier expansion of the form

$$
g(z)=\sum_{n \neq 0} b_{g, \infty}(n) W_{\frac{1}{4} \operatorname{sgn}(n), i t}(4 \pi|n| y) e(n x), \quad z=x+i y .
$$

In this section our goal is to estimate the following averages of the coefficients $b_{g, \infty}$,

$$
\sum_{X \leq n \leq 2 X}\left|b_{g, \infty}( \pm n)\right|^{2} \text { and } \sum_{X \leq n \leq 2 X}\left|b_{g, \infty}( \pm n) b_{g, \infty}( \pm n+\ell)\right| .
$$

We do this by relating the behavior of the coefficients to central values of $L$-functions through a Waldspurger type result which follows after collecting the result of Baruch 
and Mao [1] and a recent result of Duke-Imamoglu-Tóth [6] (which strengthens the earlier work of Biró [2], and Katok-Sarnak [17]). We will postpone the proof of the proposition until the appendix. We also recall the standard definition of the inner product on $\Gamma_{0}(N) \backslash \mathbb{H}$,

$$
\langle f, g\rangle_{\Gamma_{0}(N)}:=\frac{1}{\left[\mathrm{PSL}_{2}(\mathbb{Z}): \Gamma_{0}(N)\right]} \iint_{\Gamma_{0}(N) \backslash \mathbb{H}} f(z) \overline{g(z)} \cdot \frac{d x d y}{y^{2}} .
$$

Proposition 6.1. Let $g \in V^{+}$with $g \neq 0$. Suppose that $g$ is a simultaneous eigenfunction of $\Delta_{1 / 2}$ and of the Hecke operators $T_{p^{2}}, p \neq 2$. Then, there exists an even weight 0 Maaß (Hecke normalized eigen-)form $f$ with $\Delta f=-\left(\frac{1}{4}+(2 t)^{2}\right) f$ such that for any fundamental discriminant $d$,

$$
\frac{\left|b_{g, \infty}(d)\right|^{2}}{\langle g, g\rangle_{\Gamma_{0}(4)}}=\frac{L\left(\frac{1}{2}, f \otimes \chi_{d}\right)}{\langle f, f\rangle_{S L_{2}(\mathbb{Z})}} \cdot(\pi|d|)^{-1}\left|\Gamma\left(\frac{1}{2}-\frac{\operatorname{sgn}(d)}{4}-i t\right)\right|^{2} .
$$

Additionally, for an integer $n=d \delta^{2}$ with $d$ a fundamental discriminant,

$$
\left|b_{g, \infty}(n)\right|^{2} \ll_{\varepsilon}\left|b_{g, \infty}(d)\right|^{2} \cdot \delta^{2 \theta-2+\varepsilon}
$$

where $\theta$ is the best currently known exponent towards the Ramanujan conjecture in the case of weight 0 Maaß forms.

Note that in our setting $\langle g, g\rangle_{\Gamma_{0}(4)}=\frac{1}{6}$ since $g$ is normalized so that

$$
\iint_{\Gamma_{0}(4) \backslash \mathbb{H}}|g(z)|^{2} \operatorname{dvol}(z)=1
$$

In addition since the corresponding form $f$ of weight 0 is Hecke normalized, its $L^{2}$ norm is determined by the formula

$$
(\cosh 2 \pi t) \cdot\langle f, f\rangle_{\mathrm{SL}_{2}(\mathbb{Z})} \asymp L\left(1, \mathrm{Sym}^{2} f\right) .
$$

Combining the above with Proposition 6.1 and a bound towards the Ramanujan conjecture due to Kim and Sarnak [18], we obtain the following corollary.

Corollary 6.1. For $n=d \delta^{2}$ with $d$ a fundamental discriminant, we have

$$
\left|b_{g, \infty}(n)\right| \ll_{\varepsilon} \frac{1}{\sqrt{|n|}} \cdot\left(\frac{L\left(\frac{1}{2}, f \otimes \chi_{d}\right)}{L\left(1, \operatorname{Sym}^{2} f\right)}\right)^{1 / 2} \cdot|\delta|^{7 / 64+\varepsilon} \cdot|t|^{-\operatorname{sgn}(n) / 4} \cdot e^{\pi|t| / 2} .
$$

The bound of Corollary 6.1 reduces the problem of understanding (6.1) to corresponding questions on $L$-functions which we have addressed in the previous section. In this way we obtain the following two lemmas.

Lemma 6.1. Assume the Generalized Riemann Hypothesis. Then for $|t|^{\varepsilon} \leq X$

$$
\sum_{X \leq n \leq 2 X}\left|b_{g, \infty}( \pm n)\right|^{2} \ll_{\varepsilon}(\log X)^{\varepsilon} \cdot|t|^{\mp \frac{1}{2}} \cdot e^{\pi|t|} .
$$


In addition for $X \leq|t|^{\varepsilon}$ we have the trivial bound $\ll|t|^{\mp \frac{1}{2}+\varepsilon} \cdot e^{\pi|t|}$.

The trivial bound in Lemma 6.1 is a fairly direct consequence of the Generalized Riemann Hypothesis and Corollary 6.1. In the next lemma we estimate the second sum in (6.1). The proof builds in part on the argument used to establish Lemma 6.1. We notice however that a trivial application of Cauchy-Schwarz and Lemma 6.1 gives only a bound of size $|t|^{\mp \frac{1}{2}} e^{\pi|t|}(\log X)^{\varepsilon}$ for the second sum in ([6.1), which does not suffice for our argument.

Lemma 6.2. Assume the Generalized Riemann Hypothesis. Then, for $|t|^{\varepsilon} \leq X$, and $0 \neq|\ell| \leq \log X$

$$
\sum_{X \leq n \leq 2 X}\left|b_{g, \infty}( \pm n) b_{g, \infty}( \pm n+\ell)\right| \ll_{\varepsilon}|t|^{\mp \frac{1}{2}} \cdot e^{\pi|t|} \cdot(\log X)^{-25 / 156+\varepsilon} .
$$

In Lemma 6.2, we expect that the correct exponent on the logarithm is $-\frac{1}{4}$. Note that $\frac{25}{156}=\frac{1}{4}-0.089 \ldots$ and that this weaker exponent is due to the lack of the Ramanujan bound for weight 0 Maaß forms. It is however quite possible that with a more involved argument the sharp exponent $-\frac{1}{4}$ can be reached.

Proof of Lemma 6.1. Write $n=d \delta^{2}$ with $d$ a fundamental discriminant for simplicity we only consider terms with $d>0$, as those with $d<0$ are handled in the same way and satisfy the analogous bound. Let us first prove the "trivial bound". On the Generalized Riemann Hypothesis we have,

$$
\frac{L\left(\frac{1}{2}, f \otimes \chi_{d}\right)}{L\left(1, \operatorname{Sym}^{2} f\right)} \ll|t d|^{\varepsilon}
$$

Therefore, by the bound of Corollary 6.1,

$$
\begin{aligned}
& \sum_{X \leq n \leq 2 X}\left|b_{g, \infty}(n)\right|^{2} \ll \frac{|t|^{-\frac{1}{2}+\varepsilon} \cdot e^{\pi|t|}}{X} \sum_{X \leq d \delta^{2} \leq 2 X}|d|^{\varepsilon} \cdot|\delta|^{14 / 64+\varepsilon} \\
& \ll X^{\varepsilon} \cdot \frac{|t|^{-1 / 2} e^{\pi|t|}}{X} \sum_{\delta \leq \sqrt{X}}|\delta|^{16 / 64} \cdot \frac{2 X}{\delta^{2}} \ll X^{\varepsilon}|t|^{-1 / 2} e^{\pi|t|}
\end{aligned}
$$

When $X \leq|t|$ this of course implies the claimed trivial bound $|t|^{-1 / 2+\varepsilon} e^{\pi|t|}$.

Now let's consider the case when $X \geq|t|^{\varepsilon}$, in which case we can do better. Inserting the bound (6.2) we get

$$
\sum_{X \leq n \leq 2 X}\left|b_{g, \infty}(n)\right|^{2} \ll \frac{|t|^{-\frac{1}{2}} \cdot e^{\pi|t|}}{X} \sum_{X \leq d \delta^{2} \leq 2 X} \frac{L\left(\frac{1}{2}, f \otimes \chi_{d}\right)}{L\left(1, \mathrm{Sym}^{2} f\right)} \cdot \delta^{14 / 64+\varepsilon} .
$$


Pick any $0<\theta<\frac{1}{2}$. In the sum we split according to $\delta<X^{\theta}$ or $\delta>X^{\theta}$. This gives

$$
\begin{gathered}
\frac{1}{X} \sum_{X \leq d \delta^{2} \leq 2 X} \frac{L\left(\frac{1}{2}, f \otimes \chi_{d}\right)}{L\left(1, \mathrm{Sym}^{2} f\right)} \cdot \delta^{14 / 64+\varepsilon} \ll \frac{1}{X} \sum_{\delta<X^{\theta}} \delta^{14 / 64+\varepsilon} \sum_{X / \delta^{2} \leq d \leq 2 X / \delta^{2}} \frac{L\left(\frac{1}{2}, f \otimes \chi_{d}\right)}{L\left(1, \mathrm{Sym}^{2} f\right)}+ \\
+\frac{1}{X} \sum_{\delta>X^{\theta}} \delta^{14 / 64+\varepsilon} \sum_{d \leq 2 X / \delta^{2}} \frac{L\left(\frac{1}{2}, f \otimes \chi_{d}\right)}{L\left(1, \mathrm{Sym}^{2} f\right)} .
\end{gathered}
$$

We now use Lemma 5.3 to bound the sum on the RHS of (6.3) in the following way

$$
\frac{1}{X} \sum_{\delta<X^{\theta}} \delta^{14 / 64+\varepsilon} \sum_{X / \delta^{2} \leq d \leq 2 X / \delta^{2}} \frac{L\left(\frac{1}{2}, f \otimes \chi_{d}\right)}{L\left(1, \mathrm{Sym}^{2} f\right)} \ll(\log X)^{\varepsilon} \sum_{\delta<X^{\theta}} \frac{\delta^{14 / 64+\varepsilon}}{\delta^{2}} \ll(\log X)^{\varepsilon} .
$$

To estimate the sum in (6.4) note that the Lindelöf Hypothesis implies $L\left(\frac{1}{2}, f \otimes\right.$ $\left.\chi_{d}\right) / L\left(1, \operatorname{Sym}^{2} f\right) \ll(|t| d)^{\varepsilon}$. This gives

$$
\frac{1}{X} \sum_{\delta>X^{\theta}} \delta^{14 / 64+\varepsilon} \sum_{d \leq 2 X / \delta^{2}} \frac{L\left(\frac{1}{2}, f \otimes \chi_{d}\right)}{L\left(1, \operatorname{Sym}^{2} f\right)} \ll X^{\varepsilon} \sum_{\delta>X^{\theta}} \frac{\delta^{14 / 64+\varepsilon}}{\delta^{2}} \ll(\log X)^{-100} .
$$

Using this estimate in (6.4) completes the proof.

Proof of Lemma 6.2. Again let us write, $n=d_{0} \delta_{0}^{2}$ and $n+\ell=d_{1} \delta_{1}^{2}$ and as before we will only consider the case $d_{1}, d_{2}>0$. We first show that the contribution of those $n$ for which $\delta_{0}>(\log X)^{\kappa}$ or $\delta_{1}>(\log X)^{\kappa}$ is negligible, for some $0<\kappa<10$ to be fixed later. Indeed, by Cauchy-Schwarz, and Lemma 6.1, the contribution of those integers for which $\delta_{0}>(\log X)^{\kappa}$ is bounded by

$$
\left(\sum_{\substack{X \leq d \delta^{2} \leq 2 X \\ \delta>(\log X)^{\kappa}}}\left|b_{g, \infty}\left(d \delta^{2}\right)\right|^{2}\right)^{1 / 2} \cdot\left(\sum_{X / 2 \leq n \leq 3 X}\left|b_{g, \infty}(n)\right|^{2}\right)^{1 / 2} .
$$

By Lemma 6.1 the second term is $\ll|t|^{-1 / 4} \cdot e^{\frac{\pi}{2}|t|} \cdot(\log X)^{\varepsilon}$. On the other hand using (6.2) and splitting into two ranges $(\log X)^{\kappa} \leq \delta<X^{\theta}$ and $X^{\theta} \leq \delta \leq \sqrt{2 X}$, with any fixed $0<\theta<\frac{1}{2}$, we see that the sum in the first term of (6.5) is bounded by

$$
\begin{aligned}
\frac{|t|^{-\frac{1}{2}} \cdot e^{\pi|t|}}{X} & \sum_{(\log X)^{\kappa}<\delta<X^{\theta}} \delta^{14 / 64+\varepsilon} \sum_{X / \delta^{2} \leq d \leq 2 X / \delta^{2}} \frac{L\left(\frac{1}{2}, f \otimes \chi_{d}\right)}{L\left(1, \mathrm{Sym}^{2} f\right)} \\
+ & \frac{|t|^{-\frac{1}{2}} \cdot e^{\pi|t|}}{X} \sum_{\delta>X^{\theta}} \delta^{14 / 64+\varepsilon} \sum_{X / \delta^{2} \leq d \leq 2 X / \delta^{2}} \frac{L\left(\frac{1}{2}, f \otimes \chi_{d}\right)}{L\left(1, \mathrm{Sym}^{2} f\right)} .
\end{aligned}
$$


Proceeding as in the proof of Lemma 6.1 we use Lemma 5.3 to bound the first term above and the Lindelöf Hypothesis bound $L\left(\frac{1}{2}, f \otimes \chi_{d}\right) \ll(d|t|)^{\varepsilon}$ to bound the second term. This gives the following total bound for the sum in the first term of (6.5)

$$
\ll|t|^{-\frac{1}{2}} \cdot e^{\pi|t|}\left((\log X)^{\varepsilon} \sum_{\delta>(\log X)^{\kappa}} \frac{\delta^{14 / 64+\varepsilon}}{\delta^{2}}+(\log X)^{-100}\right) \ll|t|^{-\frac{1}{2}} \cdot e^{\pi|t|} \cdot(\log X)^{-25 \kappa / 32+\varepsilon} .
$$

Combining estimates, we bound (6.5) as $O\left(|t|^{-1 / 2} e^{\pi|t|}(\log X)^{-25 \kappa / 64+\varepsilon}\right)$. Similarly we can bound the contribution of those integers $n$ for which $n+\ell=d_{1} \delta_{1}^{2}$ with $d$ fundamental, and $\delta_{1}>(\log X)^{\kappa}$.

Therefore,

$$
\begin{aligned}
& \sum_{X \leq n \leq 2 X}\left|b_{g, \infty}(n) b_{g, \infty}(n+\ell)\right| \\
& \ll|t|^{-\frac{1}{2}} \cdot e^{\pi|t|}\left(\frac{(\log X)^{7 \kappa / 32+\varepsilon}}{X} \sum_{\substack{X / 2 \leq d_{0} \delta_{0}^{2} \leq 3 X \\
d_{0} \delta_{0}^{2}-d_{1} \delta_{1}^{2}=-\ell \\
1 \leq \delta_{0}, \delta_{1} \leq(\log X)^{\kappa}}} \frac{L\left(\frac{1}{2}, f \otimes \chi_{d_{0}}\right)^{1 / 2} L\left(\frac{1}{2}, f \otimes \chi_{d_{1}}\right)^{1 / 2}}{L\left(1, \operatorname{Sym}^{2} f\right)}\right. \\
& \left.+(\log X)^{-25 \kappa / 64+\varepsilon}\right) .
\end{aligned}
$$

Applying Lemmas 5.1 and 5.2 the above sum is

$$
\ll X(\log X)^{-1 / 4+\varepsilon} \sum_{1 \leq \delta_{0}, \delta_{1} \leq(\log X)^{\kappa}} \frac{1}{\left[\delta_{0}, \delta_{1}\right]^{2}} \ll X(\log X)^{-1 / 4+\varepsilon} .
$$

We conclude that

$$
\sum_{X \leq n \leq 2 X}\left|b_{g, \infty}(n) b_{g, \infty}(n+\ell)\right| \ll|t|^{-\frac{1}{2}} \cdot e^{\pi|t|} \cdot\left((\log X)^{-25 \kappa / 64}+(\log X)^{7 \kappa / 32-1 / 4}\right) \cdot(\log X)^{\varepsilon} .
$$

Choosing $\kappa=\frac{16}{39}$ we get $\ll|t|^{-\frac{1}{2}} \cdot e^{\pi|t|}(\log X)^{-25 / 156+\varepsilon}$.

\section{EXTENDing THE LENGTH OF SUMmation}

As before let $g$ be an element of a basis $g_{j}$ of $V^{+}$consisting of simultaneous eigenfunctions of $\Delta_{1 / 2}$ and $T_{p^{2}}, p \neq 2$, with each $g_{j}$ normalized so that $\iint_{\Gamma_{0}(4) \backslash \mathbb{H}}\left|g_{j}(z)\right|^{2} \operatorname{dvol}(z)=$ 1 and denote by $b_{g, \infty}(n)$ the Fourier coefficients of $g$. As usual, we write the eigenvalue of $g$ as $\lambda=-\left(\frac{1}{4}+t^{2}\right)$.

Following an idea of Holowinsky [11] we show in this section that the average of $\left|b_{g, \infty}(n)\right|^{2}$ over integers $n \asymp|t|$ can be related to a corresponding average of $\left|b_{g, \infty}(n)\right|^{2}$ 
over integers $n \asymp|t| Y$ with $Y$ a parameter whose size roughly depends on the saving in the shifted convolution problem,

$$
\sum_{n \asymp|t|} b_{g, \infty}(n) b_{g, \infty}(n+\ell)
$$

in the range $n \asymp|t|$. The advantage of such an "extension" of the length of summation will become clear in the next section, where we will show that the average of $\left|b_{g, \infty}(n)\right|^{2}$ can be estimated as soon as we sum slightly more than $|t|$ terms.

Lemma 7.1. Let $h, k$ be two smooth compactly supported functions on $\mathbb{R}_{+}$and with $k$ non-negative. Let $H(s):=\int_{0}^{\infty} h(x) x^{s-1} d x$ and $K(s):=\int_{0}^{\infty} k(x) x^{s-1} d x$ denote their respective Mellin transforms. Then, for $Y \geq 1$

$$
\begin{aligned}
K(-1) Y \mathcal{S}_{1}(0, t ; h)= & H(-1)\left(1+O\left(Y^{-1 / 2}\right)\right) \mathcal{S}_{Y}(0, t ; k)+O(\sqrt{Y}) \\
& +O_{A}\left(\frac{1}{\sqrt{Y}} \sum_{\ell \neq 0} \frac{(\nu(\ell)+1) 2^{\nu(\ell)} d(|\ell|)}{1+|\ell / Y|^{A}} \cdot \mathcal{S}_{Y}(\ell, t ; k)\right)
\end{aligned}
$$

where for a smooth, compactly supported function $\psi$, the quantity $\mathcal{S}_{Y}(\ell, t ; \psi)$ is defined as

$\int_{0}^{\infty} \psi(y Y)\left|\sum_{n \neq 0,-\ell} b_{g, \infty}(n) b_{g, \infty}(n+\ell) W_{\frac{1}{4} \operatorname{sgn}(n), i t}(4 \pi|n| y) W_{\frac{1}{4} \operatorname{sgn}(n+\ell), i t}(4 \pi|n+\ell| y)\right| \cdot \frac{d y}{y^{2}}$

and $\nu(\ell)$ is the highest power of two dividing $\ell$.

Notice here that when $\ell=0$ the term appearing inside the absolute value in $\mathcal{S}_{Y}(0, t ; \psi)$ is non-negative, since $W_{\kappa, i t}(x) \in \mathbb{R}$ for $|\kappa|<\frac{1}{2}$ and $t \in \mathbb{R}$. The proof of Lemma 7.1 is rather involved so we explain now the general principle behind its proof. Consider the incomplete Eisenstein series

$$
E(z \mid h)=\sum_{\gamma \in \Gamma_{\infty} \backslash \Gamma_{0}(4)} h(\operatorname{Im}(\gamma z))
$$

and

$$
E^{Y}(z \mid k)=\sum_{\gamma \in \Gamma_{\infty} \backslash \Gamma_{0}(4)} k(Y \cdot \operatorname{Im}(\gamma z))
$$

where $\Gamma_{\infty}$ is the stabilizer group of the cusp at infinity. In the proof of Lemma 7.1 we will evaluate

$$
\iint_{\Gamma_{0}(4) \backslash \mathbb{H}} E(z \mid h) E^{Y}(z \mid k)|g(z)|^{2} \operatorname{dvol}(z)
$$


in two different ways. First, we will express $E^{Y}(z \mid k)$ as a Perron integral involving the real analytic Eisenstein series $E(z, s)$, which we recall is defined as

$$
E(z, s)=\sum_{\gamma \in \Gamma_{\infty} \backslash \Gamma_{0}(4)}(\operatorname{Im}(\gamma z))^{s} .
$$

Shifting contours and collecting the residue from the simple pole of $E(z, s)$ at $s=1$ will lead to the main term on the left-hand side of (7.1). Second, we will use the unfolding technique with $E^{Y}(z \mid k)$ and then expand $E(z \mid h)$ as a Fourier series. This will lead to the right-hand side of (7.1). The Fourier development is described in the next lemma.

Lemma 7.2. We have

$$
E(z \mid h)=a_{0, h}(y)+\sum_{\ell \neq 0} a_{\ell, h}(y) e(\ell x)
$$

where

$$
a_{\ell, h}(y)= \begin{cases}\frac{H(-1)}{2 \pi}+O(\sqrt{y}) & \text { if } \ell=0, \\ O_{A}\left(\sqrt{y} \frac{(\nu(\ell)+1) 2^{\nu(\ell)} d(\ell)}{1+|\ell y|^{A}}\right) & \text { if } \ell \neq 0,\end{cases}
$$

and $\nu(\ell)$ is the largest power of 2 dividing $\ell$.

Proof. The proof closely follows the argument of Holowinsky [11], with appropriate modifications at the prime $p=2$. Consider the Eisenstein series

$$
E(z, s)=\sum_{\gamma \in \Gamma_{\infty} \backslash \Gamma_{0}(4)}(\operatorname{Im}(\gamma z))^{s} .
$$

At the cusp at $\infty, E(z, s)$ has the Fourier expansion

$$
E(z, s)=y^{s}+\varphi(s) y^{1-s}+2 \sqrt{y} \sum_{\ell \neq 0} \varphi(s, \ell) K_{s-\frac{1}{2}}(2 \pi|\ell| y) e(n x)
$$

here $K_{\nu}(u)$ is the modified Bessel function of second kind and for $\operatorname{Re}(s)>\frac{1}{2}$

$$
\varphi(s)=\pi^{1 / 2} \frac{\Gamma\left(s-\frac{1}{2}\right)}{4^{2 s} \Gamma(s)} \sum_{n \geq 1} \frac{\phi(4 n)}{n^{2 s}}=\pi^{1 / 2} \frac{\Gamma\left(s-\frac{1}{2}\right)}{4^{2 s} \Gamma(s)} \frac{2}{1-2^{-2 s}} \frac{\zeta(2 s-1)}{\zeta(2 s)}
$$

and

$$
\varphi(s, \ell)=\frac{\pi^{s}}{4^{2 s} \Gamma(s)}|\ell|^{s-\frac{1}{2}} \sum_{n \geq 1} \frac{c_{4 n}(\ell)}{n^{2 s}}=\frac{\pi^{s}}{4^{2 s} \Gamma(s)} \frac{\mathcal{L}_{2}(s, \ell)}{\zeta(2 s)} \sum_{a b=|\ell|}\left(\frac{a}{b}\right)^{s-\frac{1}{2}}
$$


where $c_{q}(n)$ is a Ramanujan sum (see [21, Section 2.2] and [15, Section 4.2]). Writing $\nu(\ell)$ for the largest power of 2 dividing $\ell$ note that $\mathcal{L}_{2}(s, \ell)$ is given by

$$
\mathcal{L}_{2}(s, \ell)=\frac{1-2^{1-2 s}}{\left(1-2^{(\nu(\ell)+1)(1-2 s)}\right)\left(1-2^{-2 s}\right)} \sum_{j=0}^{\nu(\ell)} \frac{c_{2^{j+2}}(\ell)}{2^{2 j s}}=O\left((\nu(\ell)+1) 2^{\nu(\ell)}\right)
$$

which holds uniformly for $\delta \leq \operatorname{Re}(s) \leq 1$, for any $\delta>0$.

To obtain the Fourier series expansion of the incomplete Eisenstein series $E(z \mid h)$ we write

$$
E(z \mid h)=\frac{1}{2 \pi i} \int_{(2)} E(z, s) H(-s) d s=a_{0}(y)+\sum_{\ell \neq 0} a_{\ell, h}(y) e(\ell x) .
$$

By shifting contours of integration it follows that

$$
a_{0, h}(y)=\frac{1}{2 \pi i} \int_{(2)}\left(y^{s}+\varphi(s) y^{1-s}\right) H(-s) d s=\frac{H(-1)}{2 \pi}+O(\sqrt{y}) .
$$

To estimate $a_{\ell, h}(y)$ we use the bounds $K_{i t}(u) \ll \min \left(u^{-1 / 2} e^{-u}, e^{-\frac{\pi}{2}|t|}\right)$ (see [8, Corollary 3.2]) and $H(-s) \ll(1+|s|)^{-A}$, to get that

$$
\begin{aligned}
a_{\ell, h}(y) & =\frac{\sqrt{y}}{2 \pi i} \int_{(2)} K_{s-\frac{1}{2}}(2 \pi|\ell| y) \frac{\pi^{s}}{4^{2 s} \Gamma(s)} \frac{\mathcal{L}_{2}(s, \ell)}{\zeta(2 s)} \sum_{a b=|\ell|}\left(\frac{a}{b}\right)^{s-\frac{1}{2}} H(-s) d s \\
& \ll \sqrt{y}(\nu(\ell)+1) 2^{\nu(\ell)} d(|\ell|) \int_{\mathbb{R}}\left|\frac{K_{i \tau}(2 \pi|\ell| y)}{\Gamma\left(\frac{1}{2}+i \tau\right)}\right|(1+|\tau|)^{-A} d \tau \\
& \ll \frac{\sqrt{y}(\nu(\ell)+1) 2^{\nu(\ell)} d(|\ell|)}{1+|\ell y|^{A}}
\end{aligned}
$$

which gives the claim.

We are now ready to prove Lemma 17.1. As promised in order to arrive at (7.1) we will evaluate (7.4) in two different ways.

7.1. Proof of Lemma 7.1 Step 1: A first evaluation of (17.4). Note that,

$$
E^{Y}(z \mid k)=\frac{1}{2 \pi i} \int_{(2)} K(-s) Y^{s} E(z, s) d s
$$

with $K(s):=\int_{0}^{\infty} k(x) x^{s-1} d x$. Therefore we can re-write (7.4) as

$$
\frac{1}{2 \pi i} \int_{(2)} K(-s) Y^{s}\left(\iint_{\Gamma_{0}(4) \backslash \mathbb{H}} E(z, s) E(z \mid h)|g(z)|^{2} \cdot \frac{d x d y}{y^{2}}\right) d s .
$$


Shifting the contour to $\operatorname{Re}(s)=\frac{1}{2}$ we collect a residue at $s=1$ coming from $E(z, s)$. The value of the residue of $E(z, s)$ at $s=1$ is $\left(\operatorname{vol}\left(\Gamma_{0}(4) \backslash \mathbb{H}\right)\right)^{-1}=(2 \pi)^{-1}$. Therefore we get that (7.4) equals

$$
\begin{aligned}
K(-1) & \cdot \frac{Y}{2 \pi} \iint_{\Gamma_{0}(4) \backslash \mathbb{H}} E(z \mid h)|g(z)|^{2} \cdot \frac{d x d y}{y^{2}} \\
& +\frac{1}{2 \pi i} \int_{(1 / 2)} K(-s) Y^{s}\left(\iint_{\Gamma_{0}(4) \backslash \mathbb{H}} E(z, s) E(z \mid h)|g(z)|^{2} \cdot \frac{d x d y}{y^{2}}\right) d s .
\end{aligned}
$$

In the first term we apply the unfolding method,

$$
\iint_{\Gamma_{0}(4) \backslash \mathbb{H}} E(z \mid h)|g(z)|^{2} \cdot \frac{d x d y}{y^{2}}=\sum_{n \neq 0}\left|b_{g, \infty}(n)\right|^{2} \int_{0}^{\infty} W_{\frac{1}{4} \operatorname{sgn}(n), i t}(4 \pi|n| y)^{2} \cdot \frac{h(y) d y}{y^{2}}
$$

In the second term, since $E(z \mid h)$ is compactly supported in $\Gamma_{0}(4) \backslash \mathbb{H}$, the $z$ variable is restricted to a compact set. Therefore we have the bound $E\left(z, \frac{1}{2}+i u\right) \ll(1+|u|)^{N}$ for some large $N$, uniformly in the compact set to which $z$ belongs, with the implied constant depending only on $h$. Therefore, since $K\left(-\frac{1}{2}-i u\right) \ll_{A}(1+|u|)^{-A}$ the integral in (7.5) is

$$
\ll \sqrt{Y} \iint_{\Gamma_{0}(4) \backslash \mathbb{H}}|E(z \mid h)||g(z)|^{2} \cdot \frac{d x d y}{y^{2}} \ll \sqrt{Y} \iint_{\Gamma_{0}(4) \backslash \mathbb{H}}|g(z)|^{2} \cdot \frac{d x d y}{y^{2}}=\sqrt{Y} .
$$

Combining this with (7.5) and (7.6) we conclude that

$$
\begin{aligned}
\iint_{\Gamma_{0}(4) \backslash \mathbb{H}} & E^{Y}(z \mid k) E(z \mid h)|g(z)|^{2} \cdot \frac{d x d y}{y^{2}} \\
& =K(-1) \frac{Y}{2 \pi} \sum_{n \neq 0}\left|b_{g, \infty}(n)\right|^{2} \int_{0}^{\infty} W_{\frac{1}{4} \operatorname{sgn}(n), i t}(4 \pi|n| y)^{2} \cdot \frac{h(y) d y}{y^{2}}+O(\sqrt{Y}) .
\end{aligned}
$$

This gives the left-hand side of (17.1).

7.2. Proof of Lemma 7.1. Step 2: A second evaluation of (17.4). By the unfolding method (7.4) is equal to

$$
\int_{0}^{\infty} \int_{-1 / 2}^{1 / 2} k(Y y) E(z \mid h)|g(z)|^{2} \cdot \frac{d x d y}{y^{2}}
$$


Expanding $E(z \mid h)$ into the Fourier series given in Lemma 7.2 we see that the previous equation equals

$$
\begin{aligned}
\int_{0}^{\infty} & \int_{-1 / 2}^{1 / 2} a_{0, h}(y) k(Y y)|g(z)|^{2} \cdot \frac{d x d y}{y^{2}} \\
& +\sum_{\ell \neq 0} \int_{0}^{\infty} \int_{-1 / 2}^{1 / 2} e(\ell x) k(Y y) a_{\ell, h}(y)|g(z)|^{2} \cdot \frac{d x d y}{y^{2}}
\end{aligned}
$$

We now investigate (7.7) and (7.8). By Lemma 7.2 the contribution of (7.7) equals

$$
\frac{H(-1)}{2 \pi} \int_{0}^{\infty} \int_{-1 / 2}^{1 / 2}|g(z)|^{2} k(Y y) \cdot \frac{d x d y}{y^{2}}+O\left(\int_{0}^{\infty} \int_{-1 / 2}^{1 / 2} \sqrt{y} k(Y y)|g(z)|^{2} \cdot \frac{d x d y}{y^{2}}\right) .
$$

Since $k$ is compactly supported we may bound the term $\sqrt{y}$ in the integrand in the second term above as $O\left(Y^{-1 / 2}\right)$. It follows from this and the non-negativity of $k$ that the above is equal to

$$
\left(1+O\left(Y^{-1 / 2}\right)\right) \frac{H(-1)}{2 \pi} \int_{0}^{\infty} \int_{-1 / 2}^{1 / 2}|g(z)|^{2} k(Y y) \cdot \frac{d x d y}{y^{2}} .
$$

Upon expanding $g(z)$ into a Fourier series we see that the above integral equals

$$
\frac{H(-1)}{2 \pi} \sum_{n \neq 0}\left|b_{g, \infty}(n)\right|^{2} \cdot \int_{0}^{\infty} W_{\frac{1}{4} \operatorname{sgn}(n), i t}(4 \pi|n| y)^{2} \cdot \frac{k(Y y) d y}{y^{2}}
$$

This gives the main term on the right-hand side of (7.1).

It remains to estimate (7.8). First, we expand $g(z)$ into a Fourier series once again to see that (7.8) is equal to

$$
\sum_{\ell \neq 0} \sum_{n \neq 0,-\ell} b_{g, \infty}(n) b_{g, \infty}(n+\ell) \int_{0}^{\infty} k(Y y) a_{\ell, h}(y) W_{\frac{1}{4} \operatorname{sgn}(n), i t}(4 \pi|n| y) W_{\frac{1}{4} \operatorname{sgn}(n+\ell), i t}(4 \pi|n+\ell| y) \cdot \frac{d y}{y^{2}} .
$$

Moving the sum over $n$ inside, taking absolute values, and applying Lemma 7.2 we see that the above is

$$
\begin{aligned}
& \ll \sum_{\ell \neq 0}(\nu(\ell)+1) 2^{\nu(\ell)} d(|\ell|) \int_{0}^{\infty} k(y Y) \cdot \frac{\sqrt{y}}{1+|\ell y|^{A}} \times \\
& \quad\left|\sum_{n \neq 0,-\ell} b_{g, \infty}(n) b_{g, \infty}(n+\ell) W_{\frac{1}{4} \operatorname{sgn}(n), i t}(4 \pi|n| y) W_{\frac{1}{4} \operatorname{sgn}(n), i t}(4 \pi|n+\ell| y)\right| \cdot \frac{d y}{y^{2}} .
\end{aligned}
$$

Since $k(\cdot)$ is compactly supported, in the integrand we may bound the term $\sqrt{y} /(1+$ $\left.|\ell y|^{A}\right)$ as $O\left(Y^{-1 / 2} \cdot\left(1+|\ell / Y|^{A}\right)^{-1}\right)$. This along with the estimate for (7.7) gives the right-hand side of (7.1) and completes the proof of Lemma 7.1. 


\section{The summation Formula And PROOF of QUE}

First recall the standard notation which will be set in place for the duration of this section: Let $g_{j}$ be a basis of $V^{+}$consisting of simultaneous eigenfunctions of $\Delta_{1 / 2}$ and $T_{p^{2}}, p \neq 2$, with each $g_{j}$ normalized so that $\int_{\Gamma_{0}(4) \backslash \mathbb{H}}\left|g_{j}(z)\right|^{2} d \operatorname{vol}(z)=1$. For simplicity we will write $g=g_{j}$ and $t=t_{j}$ so that $\Delta_{1 / 2} g=-\left(\frac{1}{4}+t^{2}\right) g$. Recall that $t \in \mathbb{R}$ since there are no forms corresponding to exceptional eigenvalues in $V^{+}$.

8.1. Estimates for Whittaker functions. Before establishing the summation formula we first establish the following estimate for Whittaker functions, which we will use repeatedly.

Lemma 8.1. Let $h$ be a smooth compactly supported function on $\mathbb{R}_{+}$. Then for $Y>0$,

$$
\int_{\mathbb{R}} W_{ \pm \frac{1}{4}, i t}(4 \pi|n| y)^{2} \cdot \frac{h(y Y)}{y^{2}} d y \ll_{\varepsilon}(1+|t|)^{ \pm \frac{1}{2}} \cdot e^{-\pi|t|} \cdot Y \cdot \begin{cases}|n /(t Y)|^{1-\varepsilon} & \text { if }|n| \leq|t| Y \\ |(t Y) / n|^{100} & \text { if }|n|>|t| Y\end{cases}
$$

Proof. The proof of (8.1) relies on some computations of Matthes [29, 30] (further refined in a lemma of Luo-Rudnick-Sarnak [27]). Let

$$
\mathcal{M}_{\kappa, i t}(s):=\int_{0}^{\infty} W_{\kappa, i t}(y)^{2} \cdot y^{s-2} d y, \operatorname{Re}(s)>0 .
$$

In Lemma 1 of [30], Matthes proves that for $s=\sigma+i \tau$ with $\varepsilon<\sigma \leq 1000$, and $-\frac{1}{2} \leq \kappa<\frac{1}{2}$

$$
\left|\mathcal{M}_{\kappa, i t}(s)\right| \leq \mathcal{M}_{\kappa, i t}(\sigma) \ll(1+|t|)^{\sigma-1+2 \kappa} e^{-\pi|t|} .
$$

In addition if $H(s):=\int_{0}^{\infty} h(y) y^{s-1} d y$, then we notice that

$$
\int_{0}^{\infty} W_{\kappa, i t}(4 \pi|n| y)^{2} \cdot \frac{h(y Y)}{y^{2}} d y=\frac{1}{2 \pi i} \int_{(-\varepsilon)} H(s) Y^{-s}(4 \pi|n|)^{s+1} \mathcal{M}_{\kappa, i t}(-s) d s .
$$

For the proof of the bound for $|n| \leq|t| Y$ we apply the triangle inequality and Matthes bound on the line $\operatorname{Re}(s)=-\varepsilon$, and for the proof of the bound for $|n|>|t| Y$ we shift to $\operatorname{Re}(s)=-101$ and then bound trivially using Matthes's result.

8.2. The summation formula. In this section we derive the analogue of a convexity bound for the Dirichlet series with coefficients given by $\left|b_{g, \infty}(n)\right|^{2}$. This allows us to estimate the average of $\left|b_{g, \infty}(n)\right|^{2}$ when we sum slightly more than $|t|$ of these coefficients. To rephrase this in terms of $L$-functions note that this is analogous to using the approximate functional equation for $L\left(\frac{1}{2}, f \otimes \chi_{d}\right)$ followed by an application of Poisson summation for $\chi_{d}$; as a result we would relate the sum of $L\left(\frac{1}{2}, f \otimes \chi_{d}\right)$ 
with $|d| \leq X$ to a a similar sum of length $1+|t|^{2} / X$, which is shorter when $X$ is slightly larger than $|t|$. From this we see that we expect to win when $X$ exceeds $|t|$ slightly. Since we can only extend our sum to have length $|t|(\log |t|)^{\eta}$ for some small $\eta>0$, we require a convexity bound which is stronger than what one obtains using the Phragmen-Lindelöf principle. For general $L$-functions such convexity bounds have been given by Heath-Brown [10], and a stronger "weakly subconvex" bound was obtained by Soundararajan [50]. The main tool used to prove the convexity bound is the following functional equation.

Lemma 8.2. Consider

$$
\mathcal{M}_{\kappa, i t}(s)=\int_{0}^{\infty} \frac{W_{\kappa, i t}(y)^{2}}{y} \cdot y^{s-1} d y \text { and } R_{ \pm, g}(s)=\sum_{n>0} \frac{\left|b_{g, \infty}( \pm n)\right|^{2}}{n^{s-1}} \text {. }
$$

Let

$$
G(s)=\sum_{ \pm} R_{ \pm, g}(s) \mathcal{M}_{ \pm \frac{1}{4}, i t}(s)
$$

Then $G(s)$ is a meromorphic function, whose only singularity in $\operatorname{Re}(s)>\frac{1}{2}$ is a simple pole at $s=1$ with residue $(2 \pi)^{-1}$. In addition $G(s)$ has no singularities in $\operatorname{Re}(s)<0$. Finally, the completed Dirichlet series $\widetilde{G}(s):=\pi^{-2 s} \Gamma(s) \zeta(2 s) G(s)$ satisfies the functional equation $\widetilde{G}(s)=\widetilde{G}(1-s)$ and $s(1-s) \widetilde{G}(s)$ is entire.

Proof. The proof of this is nearly identical to the proof given by Kohnen and Zagier [19] for holomorphic half-integral weight forms lying in the Kohnen plus space. For completeness we have included a proof in the appendix.

This allows us to prove the following "convexity bound".

Lemma 8.3. Assume the Generalized Riemann Hypothesis. Let $G(s)$ be as in Lemma 8.2 and $s=\sigma+i \tau$. Then for $\frac{1}{2}<\sigma<1$

$$
G(\sigma+i \tau) \ll_{\sigma}(\log |t|)^{\varepsilon} \cdot(1+|\tau|)^{1-\sigma+\varepsilon} .
$$

Proof. Let $H(s)=e^{s^{2}}$, and $\Lambda(s)=s(1-s) \pi^{-2 s} \Gamma(s) \zeta(2 s) G(s)$. Then according to Lemma 8.2 we have $\Lambda(s)=\Lambda(1-s)$ and $\Lambda(s)$ is an entire function. Therefore, by a standard argument, for $\operatorname{Re}(s)>\frac{1}{2}$,

$$
\Lambda(s)=\frac{1}{2 \pi i} \int_{(1)} \Lambda(s+w) \frac{H(w) d w}{w}+\frac{1}{2 \pi i} \int_{(1)} \Lambda(1-s+w) \frac{H(w) d w}{w} .
$$

Define $c_{ \pm}(n)$ by,

$$
\sum_{n \geq 1} \frac{c_{ \pm}(n)}{n^{s}}=\zeta(2 s) R_{ \pm, g}(s), c_{ \pm}(n):=\sum_{k^{2} \ell=n}\left|b_{g, \infty}( \pm \ell)\right|^{2} \ell .
$$


Also, let

$$
\Phi_{s}(w)=\frac{(s+w)(1-(s+w)) \pi^{-2 w} \Gamma(s+w)}{s(1-s)} \cdot \frac{H(w)}{w} .
$$

Thus, expanding $\zeta(2 s) R_{ \pm, g}(s)$ as a Dirichlet series we have for $\frac{1}{2}<\sigma<1$

$$
\begin{aligned}
\zeta(2 s) G(s)= & \sum_{ \pm} \sum_{n \geq 1} \frac{c( \pm n)}{n^{s}} \frac{1}{2 \pi i} \int_{(1)} n^{-w} \frac{\Phi_{s}(w)}{\Gamma(s)} \mathcal{M}_{ \pm \frac{1}{4}, i t}(s+w) d w \\
& +\sum_{ \pm} \sum_{n \geq 1} \frac{c( \pm n)}{n^{1-s}} \frac{1}{2 \pi i} \int_{(1)} n^{-w} \frac{\Phi_{1-s}(w)}{\Gamma(s)} \mathcal{M}_{ \pm \frac{1}{4}, i t}(1-s+w) d w .
\end{aligned}
$$

For $s=\sigma+i \tau$ and $w=u+i v$ Stirling's formula gives that uniformly in the range $0<\varepsilon \leq \sigma \leq A,-\sigma+\varepsilon \leq u \leq A$, and $|s-1| \geq \varepsilon$

$$
\left|\Phi_{s}(w)\right| \ll|\Gamma(s)| \cdot(1+|\tau|)^{u} e^{-v^{2} / 2} .
$$

Using this and (8.2) gives

$$
\begin{aligned}
& \int_{(1)} n^{-w} \frac{\Phi_{s}(w)}{\Gamma(s)} \mathcal{M}_{ \pm \frac{1}{4}, i t}(s+w) d w \\
& \ll \begin{cases}\left|\frac{t}{n}\right|^{c}(1+|t|)^{\sigma-1 \pm \frac{1}{2}} e^{-\pi|t|}(1+|\tau|)^{c} & \text { if }|n|>|t| \text { for any } 0<c<100, \\
(1+|t|)^{\sigma-1 \pm \frac{1}{2}} e^{-\pi|t|}\left(1+\left|\frac{t}{n}\right|^{c}\right) & \text { if }|n| \leq|t| \text { for any }-\sigma<c<0 .\end{cases}
\end{aligned}
$$

Also, as a consequence of Lemma 6.1 we have under GRH that

$$
\sum_{X \leq n \leq 2 X} c_{ \pm}(n) \ll X \cdot(1+|t|)^{\mp \frac{1}{2}} e^{\pi|t|} \cdot \begin{cases}(\log X)^{\varepsilon} & \text { if } X \geq|t|^{\varepsilon} \\ |t|^{\varepsilon} & \text { if } X \leq|t|^{\varepsilon}\end{cases}
$$

Hence, when $|n|>|t|$ we pick $c=1-\sigma+\varepsilon$ in (8.6) and using (8.7) we get

$$
\begin{aligned}
& \sum_{ \pm} \sum_{n \geq 1} \frac{c( \pm n)}{n^{s}} \frac{1}{2 \pi i} \int_{(1)} n^{-w} \frac{\Phi_{s}(w)}{\Gamma(s)} \mathcal{M}_{ \pm \frac{1}{4}, i t}(s+w) d w \\
& \ll(1+|\tau|)^{1-\sigma+\varepsilon}|t|^{\sigma-1} e^{-\pi|t|}\left(\sum_{ \pm}|t|^{ \pm \frac{1}{2}}\left(\sum_{n \leq|t|} \frac{c( \pm n)}{n^{\sigma}}+\sum_{|t| \leq n} \frac{c( \pm n)}{n^{\sigma}}\left|\frac{t}{n}\right|^{1-\sigma+\varepsilon}\right)\right) .
\end{aligned}
$$


Let $\mathcal{J}=1 /(\varepsilon \log 2)$ and apply (8.7) to see that

$$
\begin{aligned}
\sum_{n \leq|t|} \frac{c( \pm n)}{n^{\sigma}} & \ll \sum_{n \leq|t|^{\varepsilon}} \frac{c( \pm n)}{n^{\sigma}}+\frac{1}{|t|^{\sigma}} \sum_{j=1}^{\mathcal{J}} 2^{j \sigma} \sum_{\frac{|t|}{2^{j+1}} \leq n \leq \frac{|t|}{2^{j}}} c( \pm n) \\
& \ll e^{\pi|t|}|t|^{\mp \frac{1}{2}}\left(|t|^{\varepsilon}+|t|^{1-\sigma}(\log |t|)^{\varepsilon}\right) .
\end{aligned}
$$

Similarly,

$$
\begin{aligned}
\sum_{|t| \leq n} \frac{c( \pm n)}{n^{\sigma}}\left|\frac{t}{n}\right|^{1-\sigma+\varepsilon} & \ll|t|^{-\sigma} \sum_{j=0}^{\infty}\left(2^{j}\right)^{-(1+\varepsilon)} \sum_{2^{j}|t| \leq n \leq 2^{j+1}|t|} c( \pm n) \\
& \ll e^{\pi|t|}|t|^{1-\sigma \mp \frac{1}{2}}(\log |t|)^{\varepsilon} .
\end{aligned}
$$

By (8.9) and (8.10) we see that $\left(\underline{8.8)}\right.$ is $\ll(1+|\tau|)^{1-\sigma+\varepsilon}(\log |t|)^{\varepsilon}$. By a similar argument we have

$$
\sum_{ \pm} \sum_{n \geq 1} \frac{c( \pm n)}{n^{1-s}} \frac{1}{2 \pi i} \int_{(1)} n^{-w} \frac{\Phi_{1-s}(w)}{\Gamma(s)} \mathcal{M}_{ \pm \frac{1}{4}, i t}(1-s+w) d w \ll(1+|\tau|)^{1-\sigma+\varepsilon}(\log |t|)^{\varepsilon} .
$$

Using these estimates in (8.5) completes the proof.

We are now ready to prove our summation formula.

Lemma 8.4. Let $k$ be a smooth compactly supported function on $\mathbb{R}_{+}$. Then, for $Y \geq 1$,

$$
\sum_{n \neq 0}\left|b_{g, \infty}(n)\right|^{2} \int_{0}^{\infty} W_{\frac{1}{4} \operatorname{sgn}(n), i t}(4 \pi|n| y)^{2} \cdot \frac{k(y Y) d y}{y^{2}}=\frac{Y K(-1)}{2 \pi}+O\left(Y^{1 / 2+\varepsilon}(\log t)^{\varepsilon}\right) .
$$

Proof. Let us keep the notation of Lemma 8.2. Applying Mellin inversion we find that

$$
\begin{gathered}
\sum_{n \neq 0}\left|b_{g, \infty}(n)\right|^{2} \int_{0}^{\infty} W_{\frac{1}{4} \operatorname{sgn}(n), i t}(4 \pi|n| y)^{2} \cdot \frac{k(y Y) d y}{y^{2}} \\
=\frac{1}{2 \pi i} \int_{(\sigma)} K(-s) Y^{s}(4 \pi)^{1-s} G(s) d s, \sigma>1 .
\end{gathered}
$$

Shifting the contour to $\sigma=\frac{1}{2}+\varepsilon$ we collect a simple pole at $s=1$ with residue $(2 \pi)^{-1}$, and we get that the above integral equals

$$
\frac{1}{2 \pi} \cdot K(-1) Y+\frac{1}{2 \pi i} \int_{\left(\frac{1}{2}+\varepsilon\right)} K(-s) Y^{s}(4 \pi)^{1-s} G(s) d s .
$$


Applying Lemma 8.3, the above integral is

$$
O\left(Y^{\frac{1}{2}+\varepsilon}(\log |t|)^{\varepsilon}\right)
$$

which gives the claim.

8.3. Proof of Quantum Unique Ergodicity. Recall the discussion in Section 2.1] where we saw that Theorem 2 follows from the estimates

$$
\sum_{n \neq 0}\left|b_{g, \infty}(n)\right|^{2} \int_{0}^{\infty} W_{\frac{1}{4} \operatorname{sgn}(n), i t}(4 \pi y|n|)^{2} h(y) \frac{d y}{y^{2}}=\frac{1}{\operatorname{vol}\left(\Gamma_{0}(4) \backslash \mathbb{H}\right)} \int_{0}^{\infty} h(y) \frac{d y}{y^{2}}+o(1)
$$

as $|t| \rightarrow \infty$ and for $\ell \neq 0$

$\sum_{n \neq 0} b_{g, \infty}(n) b_{g, \infty}(n+\ell) \int_{0}^{\infty} W_{\frac{1}{4} \operatorname{sgn}(n), i t}(4 \pi|n| y) W_{\frac{1}{4} \operatorname{sgn}(n+\ell), i t}(4 \pi|n+\ell| y) h(y) \frac{d y}{y^{2}}=o(1)$,

as $|t| \rightarrow \infty$. To bound the off-diagonal contribution we note that $W_{\kappa, i u}(y)=$ $W_{\kappa,-i u}(y)$ and that $W_{\kappa, i u}(y) \in \mathbb{R}$. Using the bound $|a b| \leq a^{2}+b^{2}$, we see that to control the off-diagonal it will be enough to show that

$$
\sum_{n \neq 0}\left|b_{g, \infty}(n) b_{g, \infty}(n+\ell)\right| \int_{0}^{\infty} W_{\frac{1}{4} \operatorname{sgn}(n), i t}(4 \pi|n| y)^{2} \cdot \frac{h(y) d y}{y^{2}}=o(1)
$$

as $|t| \rightarrow \infty$ for $\ell \neq 0$. Thus the proof of the theorem reduces to establishing (8.12) and (8.11).

First let us consider (8.12). Combining Lemma 6.2 and Lemma 8.1 we have

$$
\begin{aligned}
& \sum_{n \neq 0}\left|b_{g, \infty}(n) b_{g, \infty}(n+\ell)\right| \int_{0}^{\infty} W_{\frac{1}{4} \operatorname{sgn}(n), i t}(4 \pi|n| y)^{2} \cdot \frac{h(y) d y}{y^{2}} \\
& \ll|t|^{ \pm \frac{1}{2}} e^{-\pi|t|}\left(\sum_{|n| \leq|t|}\left|b_{g, \infty}(n) b_{g, \infty}(n+\ell)\right|\left|\frac{n}{t}\right|^{1-\varepsilon}+\sum_{|n|>|t|}\left|b_{g, \infty}(n) b_{g, \infty}(n+\ell)\right|\left|\frac{t}{n}\right|^{100}\right) \\
& \ll(\log |t|)^{-25 / 156+\varepsilon},
\end{aligned}
$$

for each fixed $\ell \neq 0$ and in the last step the terms with $|n| \leq|t|^{\varepsilon}$ are handled trivially while in the other ranges we split up the sums into intervals of the form $\mathcal{I}_{j}=\left(|t| / 2^{j+1},|t| / 2^{j}\right], j \in \mathbb{Z}$, apply Lemma 6.2 to bound the sums over the intervals $\mathcal{I}_{j}$, then sum over $j$ (see (8.9) and (8.10) for a similar argument).

The estimation of the LHS of (8.11) is more intricate. Given smooth functions $h, k$ compactly supported on $\mathbb{R}_{+}$denote by $H(s), K(s)$ their respective Mellin transforms. 
Then, by Lemma 7.1 the LHS of (8.11) is equal to

$$
\begin{aligned}
\frac{H(-1)}{K(-1) Y} \cdot(1+ & \left.O\left(Y^{-1 / 2}\right)\right) \mathcal{S}_{Y}(0, t ; k)+O\left(Y^{-1 / 2}\right) \\
& +O\left(\frac{1}{Y^{3 / 2}} \sum_{\ell \neq 0} \frac{(\nu(\ell)+1) 2^{\nu(\ell)} d(|\ell|)}{1+\mid \ell / Y^{A}} \cdot \mathcal{S}_{Y}(\ell, t ; k)\right)
\end{aligned}
$$

where $\nu(\ell)$ is the exponent of the largest power of 2 dividing $\ell$ and $\mathcal{S}_{Y}(\ell, t ; k)$ is defined as,

$$
\int_{0}^{\infty} k(y Y)\left|\sum_{n \neq 0,-\ell} b_{g, \infty}(n) b_{g, \infty}(n+\ell) W_{\frac{1}{4} \operatorname{sgn}(n), i t}(4 \pi|n| y) W_{\frac{1}{4} \operatorname{sgn}, \mathrm{it}}(4 \pi|n+\ell| y)\right| \cdot \frac{d y}{y^{2}} .
$$

Moving the absolute value inside and using Lemma 6.2 and Lemma 8.1 we have by proceeding as in (8.13) that

$$
\left|\mathcal{S}_{Y}(\ell, t ; k)\right| \ll_{\varepsilon} Y(\log Y|t|)^{-25 / 156+\varepsilon}
$$

provided that $1 \leq Y \leq|t|$ and $\ell \leq \log |t|$. In addition applying Cauchy-Schwarz and using Lemma 6.1 and Lemma 8.1 we get

$$
\left|\mathcal{S}_{Y}(\ell, t ; k)\right| \ll_{\varepsilon} Y(\log Y|t|)^{\varepsilon}
$$

for all $\ell$. Choosing $Y=(\log |t|)^{\eta}$ for some exponent $\eta<1$ to be specified later it follows that the error term in (8.14) is

$$
\ll Y^{1 / 2} \cdot(\log |t|)^{-25 / 156+\varepsilon} .
$$

To estimate the main term in (8.14) apply the summation formula in Lemma 8.4 to see that

$$
\frac{H(-1)}{K(-1) Y} \mathcal{S}_{Y}(0, t ; k)=\frac{H(-1)}{2 \pi}+O\left(Y^{-1 / 2+\varepsilon}(\log t)^{\varepsilon}\right) .
$$

Using (8.15) and (8.16) in (8.14) it follows that

$$
\begin{aligned}
\sum_{n \neq 0}\left|b_{g, \infty}(n)\right|^{2} & \int_{0}^{\infty} W_{\frac{1}{4} \operatorname{sgn}(n), i t}(4 \pi y|n|)^{2} h(y) \frac{d y}{y^{2}} \\
& =\frac{H(-1)}{2 \pi}+O\left(Y^{-1 / 2+\varepsilon}(\log |t|)^{\varepsilon}\right)+O\left(\sqrt{Y}(\log |t|)^{-25 / 156+\varepsilon}\right) .
\end{aligned}
$$

Choosing $Y=(\log |t|)^{25 / 156}$ the error term is $O\left((\log |t|)^{-25 / 312+\varepsilon}\right)=o(1)$, thereby establishing (8.11). Additionally, we have already shown that the LHS of (8.12) is $O\left((\log |t|)^{-25 / 156+\varepsilon}\right)$. Therefore, we have proved Theorem 2 . 


\section{Equidistribution OF MASS AND ZEROS OF HALF-INTEGRAL WEIGHT HOLOMORPHIC FORMS}

9.1. Preliminaries. Write $S_{k+1 / 2}\left(\Gamma_{0}(4)\right)$ for the space of weight $k+\frac{1}{2}$ holomorphic cusp forms for $\Gamma_{0}(4)$. Every $g \in S_{k+1 / 2}\left(\Gamma_{0}(4)\right)$ has a Fourier expansion of the form

$$
g(z)=\sum_{n \geq 1} c(n) e(n z)
$$

and for odd $p$ the Hecke operator $T_{p^{2}}$ is defined on $S_{k+1 / 2}\left(\Gamma_{0}(4)\right)$ as

$$
T_{p^{2}} g(z)=\sum_{n \geq 1}\left(c\left(p^{2} n\right)+\left(\frac{(-1)^{k} n}{p}\right) p^{k-1} c(n)+p^{2 k-1} c\left(\frac{n}{p^{2}}\right)\right) e(n z) .
$$

Let us now recall some results of Kohnen [20] and Kohnen-Zagier [19]. The Kohnen plus space $S_{k+1 / 2}^{+}\left(\Gamma_{0}(4)\right)$ denotes the subspace of $S_{k+1 / 2}\left(\Gamma_{0}(4)\right)$ of cusp forms whose Fourier coefficients satisfy $c(n)=0$ unless $(-1)^{k} n \equiv 0,1(\bmod 4)$ and has a basis consisting of simultaneous eigenfunctions of the operators $T_{p^{2}}$ for all odd $p$. For such a $g \in S_{k+1 / 2}^{+}\left(\Gamma_{0}(4)\right)$ with $T_{p^{2}} g=\lambda_{g}(p) g$ there exists a Hecke cusp form $f \in S_{2 k}\left(\mathrm{SL}_{2}(\mathbb{Z})\right)$, such that $H_{p} f=\lambda_{g}(p) f$ where $H_{p}$ is the usual Hecke operator for $S_{2 k}\left(\mathrm{SL}_{2}(\mathbb{Z})\right)$. By the strong multiplicity one theorem this determines $f$ (up to scalar multiplication). In addition, writing the Fourier expansion of $f$ as

$$
f(z)=\sum_{n \geq 1} a(n) e(n z)
$$

and normalizing with $a(1)=1$ the Fourier coefficients of $f$ and $g$ are related by the formula

$$
c\left(|d| \delta^{2}\right)=c(|d|) \sum_{e \mid \delta} \mu(e) e^{k-1} \chi_{d}(e) a\left(\frac{\delta}{e}\right)
$$

where $d$ is a fundamental discriminant with $(-1)^{k} d>0$. With this correspondence, one has for $n=\delta^{2}|d|$ with $d$ a fundamental discriminant such that $(-1)^{k} d>0$

$$
|c(n)| \ll n^{\frac{k}{2}-\frac{1}{4}} \delta^{\varepsilon}(4 \pi)^{k / 2}\left(\frac{1}{\Gamma\left(k+\frac{1}{2}\right)}\right)^{1 / 2}\left(\frac{L\left(\frac{1}{2}, f \otimes \chi_{d}\right)}{L\left(1, \mathrm{Sym}^{2} f\right)}\right)^{1 / 2},
$$

where $g$ has been $L^{2}$-normalized, that is,

$$
\iint_{\Gamma_{0}(4) \backslash \mathbb{H}} y^{k+1 / 2}|g(z)|^{2} \operatorname{dvol}(z)=1,
$$

and recall $f$ is arithmetically normalized with $a(1)=1$ so that

$$
\iint_{\mathrm{SL}_{2}(\mathbb{Z}) \backslash \mathbb{H}} y^{2 k}|f(z)|^{2} \operatorname{dvol}(z)=\frac{(2 k-1) ! L\left(1, \mathrm{Sym}^{2} f\right)}{(4 \pi)^{2 k-1} 2 \pi^{2}} .
$$


Kohnen-Zagier also showed that the function

$$
D(s)=(4 \pi)^{-k-\frac{1}{2}} \Gamma\left(s+k-\frac{1}{2}\right) \sum_{n \geq 1} \frac{|c(n)|^{2}}{n^{s+k-\frac{1}{2}}}
$$

is absolutely convergent for $\operatorname{Re}(s)>1$, admits a meromorphic continuation to the complex plane with the only singularity in $\operatorname{Re}(s) \geq \frac{1}{2}$ being a simple pole at $s=1$ with residue $1 /(2 \pi)$ (note that $D(s)$ may have poles at $s=\rho / 2$ for zeros $\rho$ of $\zeta(s)$ ). Moreover, the completed function $\widetilde{D}(s)=\pi^{-2 s} \Gamma(s) \zeta(2 s) D(s)$ satisfies the functional equation $\widetilde{D}(s)=\widetilde{D}(1-s)$ and $s(1-s) \widetilde{D}(s)$ is an entire function.

Finally we record the simple consequence of Stirling's formula which will be used repeatedly

$$
\frac{\Gamma(s+k)}{\Gamma(k)}=k^{s}\left(1+O\left((|s|+1)^{2} k^{-1}\right)\right)
$$

for $|s|=o(\sqrt{k})$.

9.2. Proof of Theorem 1. It suffices to show that

$$
\sum_{n \geq 1}|c(n)|^{2} \int_{0}^{\infty} e^{-4 \pi n y} y^{k+\frac{1}{2}} h(y) \frac{d y}{y^{2}}=\frac{1}{\operatorname{vol}\left(\Gamma_{0}(4) \backslash \mathbb{H}\right)} \int_{0}^{\infty} h(y) \frac{d y}{y^{2}}+o(1)
$$

as $k \rightarrow \infty$ and for $\ell \neq 0$

$$
\sum_{n \geq 1} c(n) c(n+\ell) \int_{0}^{\infty} e^{-2 \pi(2 n+\ell) y} y^{k+\frac{1}{2}} h(y) \frac{d y}{y^{2}}=o(1)
$$

as $k \rightarrow \infty$ (see Section 2.1).

To estimate the sums of Fourier coefficients we will proceed in the same way as for the Maaß forms. First, we will use estimates for moments of $L\left(\frac{1}{2}, f \otimes \chi_{d}\right)$ and (9.1) to obtain (9.4). Next, to estimate the main term (9.3) we will extend the length of summation then apply a summation formula, which is obtained through a convexity bound for $D(s)$. This will give (9.3) thereby proving Theorem 1 .

9.2.1. Estimates for moments. Let $f$ be a weight $2 k$ level 1 holomorphic Hecke cusp form. We require analogues of Lemmas 5.1 and 5.3. Here the weight plays the role of the spectral parameter $|t|$. Additionally, since we have Deligne's bound for the Hecke eigenvalues of $f$ it follows from GRH that

$$
\frac{1}{\log \log k} \ll L\left(1, \operatorname{Sym}^{2} f\right) \ll \log \log k .
$$


Repeating the same argument used in the proof of Lemma 5.1 we have uniformly for $X>k^{\varepsilon}$ and $1 \leq a, b,|\ell| \leq(\log X)^{100}$ that

$$
\sum_{\substack{d_{1}, d_{2} \\ a\left|d_{1}\right| \leq X \\ a d_{1}=b d_{2}+\ell}} L\left(\frac{1}{2}, f \otimes \chi_{d_{1}}\right)^{1 / 2} L\left(\frac{1}{2}, f \otimes \chi_{d_{2}}\right)^{1 / 2} \ll \frac{X}{[a, b]}(\log X)^{-1 / 4+\varepsilon}
$$

and

$$
\sum_{|d| \leq X} L\left(\frac{1}{2}, f \otimes \chi_{d}\right) \ll X(\log X)^{\varepsilon},
$$

where the summation in both sums is over fundamental discriminants. In fact, in the holomorphic case the estimate (9.5) allows us to omit entirely the argument used in the proof of Lemma 5.3, where we treated the small primes separately.

From the moments estimates we obtain a bound on sums of Fourier coefficients. Using (9.1) in place of Corollary 6.1 we proceed as in Section 6 to see that for $X \geq k^{\varepsilon}$ that

$$
\sum_{X \leq n \leq 2 X} \frac{|c(n) c(n+\ell)|}{(2 \pi(2 n+\ell))^{k-\frac{1}{2}}} \ll \frac{X}{\Gamma\left(k+\frac{1}{2}\right)} \cdot \begin{cases}(\log X)^{-1 / 4+\varepsilon} & \text { if } 0 \neq|\ell| \leq \log X \\ (\log X)^{\varepsilon} & \text { if } \ell=0 .\end{cases}
$$

Here one uses the elementary bound

$$
\left(\frac{\sqrt{n(n+\ell)}}{n+\ell / 2}\right)^{k-\frac{1}{2}} \leq 1
$$

which holds for $|\ell|<n / 2$. Also, it follows from the Lindelöf bound that the LHS of (9.6) is $\ll(X k(|\ell|+1))^{\varepsilon} X / \Gamma\left(k+\frac{1}{2}\right)$ for all $X \geq 1$ and $\ell$.

9.2.2. Extending the length of summation. Next, we need an analogue of Lemma 7.1. Let $h, j \in C_{c}^{\infty}\left(\mathbb{R}_{+}\right), j(x) \geq 0$, with Mellin transforms $H(s), J(s)$ (resp.). As before, we evaluate

$$
\iint_{\Gamma_{0}(4) \backslash \mathbb{H}} E(z \mid h) E^{Y}(z \mid j) y^{k+\frac{1}{2}}|g(z)|^{2} \operatorname{dvol}(z)
$$

in two different ways. Here $E(z \mid h)$ and $E^{Y}(z \mid j)$ are as defined in (17.2) and (7.3) (resp.). First, we proceed as in Section 7.1 rewriting $E^{Y}(z \mid j)$ in terms of $E(z, s)$, using Mellin inversion, then shifting contours of integration (taking advantage of the analytic continuation of the Eisenstein series) to see that (9.7) equals

$$
J(-1) \frac{Y}{2 \pi} \sum_{n \geq 1}|c(n)|^{2} \int_{0}^{\infty} e^{-4 \pi n y} \cdot y^{k-\frac{3}{2}} h(y) d y+O(\sqrt{Y}) .
$$


Next, as in Section 7.2 we use the unfolding technique with $E^{Y}(z \mid j)$ and then expand $E(z \mid h)$ into a Fourier series as given in Lemma 7.2 to see that (9.7) equals

$$
\sum_{n, \ell} c(n) c(n+\ell) \int_{0}^{\infty} a_{\ell, h}(y) e^{-2 \pi(2 n+\ell) y} y^{k-\frac{3}{2}} j(y Y) d y .
$$

Applying the estimates for $a_{\ell, h}(y)$ given in Lemma 7.2 we get that the above equals

$$
\frac{H(-1)}{2 \pi} \sum_{n \geq 1}|c(n)|^{2} \int_{0}^{\infty} e^{-4 \pi n y} \cdot y^{k-\frac{3}{2}} j(y Y) d y \cdot\left(1+\left(Y^{-1 / 2}\right)\right)+O\left(\mathcal{E}_{Y}\right)
$$

where

$$
\mathcal{E}_{Y}=\frac{1}{\sqrt{Y}} \sum_{\ell \neq 0} \frac{(\nu(\ell)+\ell) 2^{\nu(\ell)} d(|\ell|)}{1+|\ell / Y|^{A}} \sum_{n \geq 1}|c(n) c(n+\ell)| \int_{0}^{\infty} e^{-2 \pi(2 n+\ell) y} \cdot y^{k-\frac{3}{2}} j(y Y) d y
$$

and $\nu(\ell)$ is the larges power of 2 dividing $\ell$. This completes our second evaluation of (9.7). Combining the above formulas gives us the extended sum

$$
\begin{aligned}
& H(-1) \sum_{n \geq 1}|c(n)|^{2} \int_{0}^{\infty} e^{-4 \pi n y} \cdot y^{k-\frac{3}{2}} j(Y y) d y \cdot\left(1+\left(Y^{-1 / 2}\right)\right) \\
& \quad=J(-1) Y \sum_{n \geq 1}|c(n)|^{2} \int_{0}^{\infty} e^{-4 \pi n y} \cdot y^{k-\frac{3}{2}} h(y) d y+O\left(\mathcal{E}_{Y}\right)+O(\sqrt{Y}) .
\end{aligned}
$$

9.2.3. A summation formula. We will show assuming GRH that for $\frac{1}{2}<\sigma<1$

$$
|D(\sigma+i \tau)| \ll(1+|\tau|)^{1-\sigma+\varepsilon}(\log k)^{\varepsilon} .
$$

By a contour integration argument this gives the following summation formula

$$
\begin{aligned}
\sum_{n \geq 1}|c(n)|^{2} \int_{0}^{\infty} e^{-4 \pi n y} \cdot y^{k-\frac{3}{2}} j(Y y) d y & =\frac{1}{2 \pi i} \int_{(2)} J(-s) Y^{s}(4 \pi)^{1-s} D(s) d s \\
& =\frac{Y J(-1)}{2 \pi}+O\left(Y^{1 / 2+\varepsilon}(\log k)^{\varepsilon}\right) .
\end{aligned}
$$

To show (9.9) let

$$
\sum_{n \geq 1} \frac{\widetilde{c}(n)}{n^{s}}=\zeta(2 s) \sum_{n \geq 1} \frac{|c(n)|^{2}}{n^{s+k-\frac{1}{2}}}, \quad \widetilde{c}(n)=\sum_{m^{2} h=n}|c(h)|^{2} h^{\frac{1}{2}-k}
$$

and note that by (9.6) we have

$$
\sum_{X \leq n \leq 2 X} \widetilde{c}(n) \ll \frac{(4 \pi)^{k} X}{\Gamma\left(k+\frac{1}{2}\right)} \cdot \begin{cases}(\log k)^{\varepsilon} & \text { if } X>k^{\varepsilon} \\ k^{\varepsilon} & \text { if } X \leq k^{\varepsilon}\end{cases}
$$




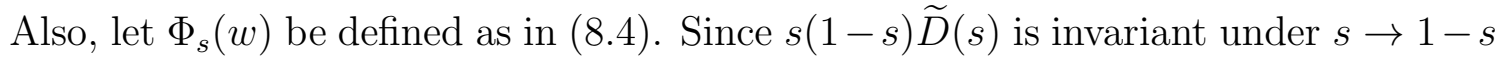
a standard contour integration argument gives

$$
\begin{aligned}
(4 \pi)^{k+1 / 2} \zeta(2 s) D(s)= & \sum_{n \geq 1} \frac{\widetilde{c}(n)}{n^{s}} \frac{1}{2 \pi i} \int_{(1)} n^{-w} \Phi_{s}(w) \frac{\Gamma\left(k+w+s-\frac{1}{2}\right)}{\Gamma(s)} d w \\
& +\sum_{n \geq 1} \frac{\widetilde{c}(n)}{n^{1-s}} \frac{1}{2 \pi i} \int_{(1)} n^{-w} \Phi_{1-s}(w) \frac{\Gamma\left(k+w-s+\frac{1}{2}\right)}{\Gamma(s)} d w .
\end{aligned}
$$

Using an analogue of (8.6) (which is obtained by applying (9.2)) along with (9.11) we see that

$$
\begin{aligned}
& \frac{1}{(4 \pi)^{k+1 / 2}} \sum_{n \geq 1} \frac{\widetilde{c}(n)}{n^{s}} \int_{(1)} n^{-w} \Phi_{s}(w) \frac{\Gamma\left(k+s+w-\frac{1}{2}\right)}{\Gamma(s)} d w \\
& \ll(1+|\tau|)^{1-\sigma+\varepsilon}(4 \pi)^{-k} k^{\sigma-1} \Gamma\left(k+\frac{1}{2}\right)\left(\sum_{n \leq k} \frac{\widetilde{c}(n)}{n^{\sigma}}+\sum_{n \geq k} \frac{\widetilde{c}(n)}{n^{\sigma}}\left|\frac{k}{n}\right|^{1-\sigma+\varepsilon}\right) \\
& \ll(\log k)^{\varepsilon}(1+|\tau|)^{1-\sigma+\varepsilon} .
\end{aligned}
$$

Similarly, the second term in (9.12) is $O\left((1+|\tau|)^{1-\sigma+\varepsilon}(\log k)^{\varepsilon}\right)$. This proves (9.9).

9.2.4. Completion of the proof. First we will establish (9.4). Note that for $h \in$ $C_{c}^{\infty}\left(\mathbb{R}_{+}\right)$using Mellin inversion together with (9.2) then applying (9.6) we have that

$$
\begin{aligned}
& \sum_{n \geq 1} c(n) c(n+\ell) \int_{0}^{\infty} e^{-2 \pi(2 n+\ell) y} y^{k+\frac{1}{2}} h(y) \frac{d y}{y^{2}} \\
& \quad \ll \Gamma\left(k-\frac{1}{2}\right) \sum_{n \geq 1} \frac{|c(n) c(n+\ell)|}{(2 \pi(2 n+\ell))^{k-\frac{1}{2}}}\left(\left|h\left(\frac{k-\frac{1}{2}}{(2 \pi(2 n+\ell))}\right)\right|+\frac{1}{k^{1-\varepsilon}} \cdot \frac{1}{1+(n / k)^{A}}\right) \\
& \ll \frac{\Gamma\left(k-\frac{1}{2}\right)}{\Gamma\left(k+\frac{1}{2}\right)} \cdot k(\log k)^{-1 / 4+\varepsilon} \ll(\log k)^{-1 / 4+\varepsilon} .
\end{aligned}
$$

It remains to estimate the main term (9.3) and to accomplish this we will use (9.8). We first estimate $\mathcal{E}_{Y}$ by choosing $Y=(\log k)^{\eta}$ for some $0<\eta<1$ to be chosen later 
and proceeding as above we get that

$$
\begin{aligned}
& \mathcal{E}_{Y}= \frac{1}{\sqrt{Y}} \sum_{\ell \neq 0} \sum_{\ell \neq 0} \frac{(\nu(\ell)+\ell) 2^{\nu(\ell)} d(|\ell|)}{1+|\ell / Y|^{A}} \sum_{n \geq 1}|c(n) c(n+\ell)| \int_{0}^{\infty} j(y Y) e^{-2 \pi(2 n+\ell) y} y^{k-3 / 2} d y \\
& \ll \frac{\Gamma\left(k-\frac{1}{2}\right)}{\sqrt{Y}} \sum_{\ell \neq 0} \frac{(\nu(\ell)+1) 2^{\nu(\ell)} d(|\ell|)}{1+|\ell / Y|^{A}} \sum_{n \geq 1} \frac{|c(n) c(n+\ell)|}{(2 \pi(2 n+\ell))^{k-\frac{1}{2}}} \\
& \times\left(j\left(\frac{Y\left(k-\frac{1}{2}\right)}{(2 \pi(2 n+\ell))}\right)+\frac{1}{k^{1-\varepsilon}} \cdot \frac{1}{1+(n /(k Y))^{A}}\right) \\
& \ll Y^{3 / 2}(\log k)^{-1 / 4+\varepsilon},
\end{aligned}
$$

where to handle the contribution of the terms with $\log k<\ell<k$ we used CauchySchwarz and the second bound in (9.6) and for $\ell>k$ we use instead the trivial bound. Combining this along with (9.8) and (9.10) gives

$\sum_{n \geq 1}|c(n)|^{2} \int_{0}^{\infty} e^{-4 \pi n y} \cdot y^{k-\frac{3}{2}} h(y) d y=\frac{H(-1)}{2 \pi}+O\left(Y^{-1 / 2+\varepsilon}(\log k)^{\varepsilon}\right)+O\left(\sqrt{Y}(\log k)^{-1 / 4+\varepsilon}\right)$.

Taking $Y=(\log k)^{1 / 4}$ we get

$$
\sum_{n \geq 1}|c(n)|^{2} \int_{0}^{\infty} e^{-4 \pi n y} \cdot y^{k-\frac{3}{2}} h(y) d y=\frac{1}{\operatorname{vol}\left(\Gamma_{0}(4) \backslash \mathbb{H}\right)} \int_{0}^{\infty} h(y) \frac{d y}{y^{2}}+O\left((\log k)^{-1 / 8+\varepsilon}\right),
$$

thereby establishing (9.3) and completing the proof of Theorem 1 .

9.3. Equidistribution of zeros: Proof of Corollary 1.1. For a holomorphic function $f$ on $\mathbb{H}$ let $\operatorname{ord}_{\varrho}(f)$ denote the order of vanishing of $f$ at $\varrho$. Also let

$$
\Gamma_{z}=\left\{\gamma \in \Gamma_{0}(4) /\{ \pm 1\}: \gamma z=z\right\}
$$

be the stabilizer group of $z$ and write $M_{k+1 / 2}\left(\Gamma_{0}(4)\right)$ for the space of weight $k+$ $1 / 2$ holomorphic modular forms for $\Gamma_{0}(4)$. For $g \in M_{k+1 / 2}\left(\Gamma_{0}(4)\right)$ one trivially has $f(z):=g(z)^{4} \in M_{4 k+2}\left(\Gamma_{0}(4)\right)$ (notice that $\left(\left(\frac{-1}{d}\right)^{k} \overline{\varepsilon_{d}}\left(\frac{c}{d}\right)^{2 k+1}\right)^{4}=1$ ). The standard valence formula (see [40, 15]) applied to $f$ gives

$$
N_{f}:=\sum_{\mathfrak{a}} \operatorname{ord}_{\mathfrak{a}}(f)+\sum_{\varrho \in \Gamma_{0}(4) \backslash \mathbb{H}} \frac{1}{\# \Gamma_{\varrho}} \cdot \operatorname{ord}_{\varrho}(f)=\left[\mathrm{SL}_{2}(\mathbb{Z}): \Gamma_{0}(4)\right] \cdot \frac{(4 k+2)}{12}
$$

where the first sum is over the cusps $\mathfrak{a}$ of $\Gamma_{0}(4) \backslash \mathbb{H}$ and $\operatorname{ord}_{\mathfrak{a}}(f)$ is the order of vanishing of $f$ at $\mathfrak{a}$. Since $\operatorname{ord}_{\varrho}(f)=4 \operatorname{ord}_{\varrho}(g)$ we conclude that the total number of weighted $\Gamma_{0}(4)$ nonequivalent zeros of $g$, which we denote by $N_{g}$, equals

$$
N_{g}=\frac{1}{2} \cdot\left(k+\frac{1}{2}\right)
$$


for any modular form $g \in M_{k+1 / 2}\left(\Gamma_{0}(4)\right)$.

Given $\psi \in C_{c}^{\infty}(\mathbb{H})$ define

$$
\Psi(z)=\sum_{\gamma \in \Gamma_{0}(4)} \psi(\gamma z)
$$

so that $\Psi(z) \in C_{c}^{\infty}\left(\Gamma_{0}(4) \backslash \mathbb{H}\right)$. Again, for $g \in M_{k+1 / 2}\left(\Gamma_{0}(4)\right)$ we note that $f(z)=$ $g(z)^{4} \in M_{4 k+2}\left(\Gamma_{0}(4)\right)$ so Lemma 2.1 of Rudnick [38] applies to $f$, from which we deduce that for any $g \in M_{k+1 / 2}\left(\Gamma_{0}(4)\right)$

$$
\begin{aligned}
\frac{1}{N_{g}} \sum_{\varrho \in \Gamma_{0}(4) \backslash \mathbb{H}} \frac{1}{\# \Gamma_{\varrho}} \cdot \operatorname{ord}_{\varrho} g(\varrho) \Psi(\varrho)= & \frac{1}{\operatorname{vol}\left(\Gamma_{0}(4) \backslash \mathbb{H}\right)} \iint_{\Gamma_{0}(4) \backslash \mathbb{H}} \Psi(z) \frac{d x d y}{y^{2}} \\
& +\frac{2}{\left(k+\frac{1}{2}\right) \pi} \iint_{\Gamma_{0}(4) \backslash \mathbb{H}} \log \left(y^{k+1 / 2}|g(z)|^{2}\right) \Delta_{\mathbb{H}} \Psi(z) \frac{d x d y}{y^{2}} .
\end{aligned}
$$

The first integral above is our main term and it remains to estimate the second integral (here we will assume additional properties about $g$ ). Let $\left\{g_{k}\right\}$ be a sequence of weight $k+1 / 2$ cusp forms lying in the Kohnen plus subspace that are also eigenfunctions of $T_{p^{2}}$ for all $p>2$. Corollary 1.1 follows from the estimate

$$
\left|\frac{1}{k} \iint_{\Gamma_{0}(4) \backslash \mathbb{H}} \log \left(y^{k+1 / 2}\left|g_{k}(z)\right|^{2}\right) \Delta_{\mathbb{H}} \Psi(z) \frac{d x d y}{y^{2}}\right|=o(1) \quad(k \rightarrow \infty) .
$$

Note that we can and will assume that $g_{k}$ is $L^{2}$-normalized.

To establish (9.14) one can follow either Rudnick's argument (see also the earlier proof of Shiffman-Zelditch [41]) or the argument used to prove Theorem 2.1 of [22]. Both extend to a more general setting and establish (9.14) provided that the following two criteria are met:

i) Inside compact subsets of $\Gamma_{0}(4) \backslash \mathbb{H}$ one has $y^{k+1 / 2}\left|g_{k}(z)\right|^{2} \ll k^{A}$ for some $A>0$.

ii) For each hyperbolic ball $B(r, z) \subset \Gamma_{0}(4) \backslash \mathbb{H}$ there exists a point $z_{0}=x_{0}+i y_{0} \in$ $B(r, z)$ such that given $\varepsilon>0$ one has for all sufficiently large $k$ that

$$
y_{0}^{k+1 / 2}\left|g_{k}\left(z_{0}\right)\right|^{2} \geq e^{-k \varepsilon} \text {. }
$$

Following an argument of Iwaniec and Sarnak (see Lemma A.1 of [13]), Rudnick (see Appendix A.2) showed $i$ ) holds for integral weight holomorphic forms with $A=1$ and a straightforward adaptation of this method gives $i$ ) for any $g \in M_{k+1 / 2}\left(\Gamma_{0}(4)\right)$. Moreover, for $g_{k}$ as above Steiner [51] proved that $i$ ) holds with $A=\frac{6}{7}+\varepsilon$ unconditionally and with $A=\frac{1}{2}+\varepsilon$ under GRH. To establish $i i$ ) we apply Theorem 1, which gives a much better lower bound. This is the only place where QUE is used in this argument. Since criteria $i$ ) and $i i$ ) hold the methods of either Rudnick and 
Shiffman-Zelditch, or, [22] give (9.14). Thus, by (9.13) and (9.14), along with an approximation argument, we conclude that for a compact subset $\mathcal{D} \subset \Gamma_{0}(4) \backslash \mathbb{H}$ with boundary measure zero we have as $k \rightarrow \infty$

$$
\frac{1}{N_{g_{k}}} \sum_{\varrho \in \mathcal{D}} \frac{1}{\# \Gamma_{\varrho}} \cdot \operatorname{ord}_{\varrho}\left(g_{k}\right)=\frac{\operatorname{vol}(\mathcal{D})}{\operatorname{vol}\left(\Gamma_{0}(4) \backslash \mathbb{H}\right)}+o(1)
$$

thereby proving Corollary 1.1. Finally, note the above result shows that $\operatorname{ord}_{\varrho}\left(g_{k}\right)=$ $o(k)$, so since $\# \Gamma_{z}=1$ for all but $O(1)$ points it follows that we can restate this as

$$
\sum_{\substack{o \in \mathcal{D} \\ g_{k}(\rho)=0}} 1=\frac{k}{2} \cdot \frac{\operatorname{vol}(\mathcal{D})}{\operatorname{vol}\left(\Gamma_{0}(4) \backslash \mathbb{H}\right)}+o(k)
$$

where in the sum the zeros are counted with multiplicity.

\section{Appendix: Proof of Proposition 6.1}

The formula for $\left|b_{g, \infty}(d)\right|$ with $d$ a fundamental discriminant follows from the main result of Baruch and Mao [1]. Given a fundamental discriminant $d$, consider the $d$ th Shimura lift,

$$
\operatorname{Sh}_{d} g(z)=\sqrt{y} \sum_{k \neq 0} 2 a_{\mathrm{Sh}_{d} g}(|k|) K_{2 i t}(2 \pi|k| y) e(k x)
$$

where

$$
a_{\mathrm{Sh}_{d} g}(k)=k \sum_{\substack{m n=k \\ m, n>0}} \frac{1}{n^{3 / 2}}\left(\frac{d}{n}\right) b_{g, \infty}\left(d m^{2}\right) .
$$

If $\operatorname{Sh}_{d} g(z)$ is identically equal to zero, then by Möbius inversion $b_{g, \infty}\left(d \delta^{2}\right)=0$ for all $\delta>0$ and therefore the bound $\left|b_{g, \infty}\left(d \delta^{2}\right)\right|^{2} \ll\left|b_{g, \infty}(d)\right|^{2} \delta^{2 \theta-2+\varepsilon}$ is vacuously true for that fundamental discriminant $d$ and all integers $\delta>0$. On the other hand if $\operatorname{Sh}_{d} g(z)$ is not identically zero, then following the proof of Proposition 6 in [6] we conclude that $\operatorname{Sh}_{d} g(z)$ is a weight 0 Maaß form. Therefore there exists a Hecke normalized Hecke-Maaß form $\phi$ of weight zero, such that $\left\langle\mathrm{Sh}_{d} g, \phi\right\rangle \neq 0$ (where $\langle\cdot, \cdot\rangle$ corresponds to the Petersson inner product. Consider now the weight 0 Hecke operators $H_{p}$. These are self-adjoint for $\langle\cdot, \cdot\rangle$ and commute with the Shimura lift, in the sense that $H_{p}\left(\mathrm{Sh}_{d} g(z)\right)=\mathrm{Sh}_{d}\left(T_{p^{2}} g(z)\right)$ for all primes $p>2$. Let $\lambda_{p}$ denote the $T_{p^{2}}$ eigenvalues of $g$, so that $T_{p^{2}} g=\lambda_{p} g$ for all $p>2$, and denote by $\lambda_{\phi}(p)$ the Hecke eigenvalues of $\phi$, so that $H_{p} \phi=\lambda_{\phi}(p) \phi$ for all primes $p$. Using the just mentioned two facts about the Hecke operators $H_{p}$, we find that for all $p>2$,

$$
\lambda_{p}\left\langle\mathrm{Sh}_{d} g, \phi\right\rangle=\left\langle\mathrm{Sh}_{d}\left(T_{p^{2}} g\right), \phi\right\rangle=\left\langle H_{p}\left(\mathrm{Sh}_{d} g\right), \phi\right\rangle=\left\langle\mathrm{Sh}_{d} g, H_{p} \phi\right\rangle=\left\langle\mathrm{Sh}_{d} g, \phi\right\rangle \cdot \lambda_{\phi}(p) .
$$

Since $\left\langle\mathrm{Sh}_{d} g, \phi\right\rangle \neq 0$ we conclude that $\lambda_{p}=\lambda_{\phi}(p)$ for all $p>2$, hence $T_{p^{2}} g=\lambda_{\phi}(p) g$ for all $p>2$. Taking the Shimura lift on both sides of this equation, and using the 
relation $H_{p}\left(\mathrm{Sh}_{d} g(z)\right)=\mathrm{Sh}_{d}\left(T_{p^{2}} g(z)\right)$, we conclude that $H_{p}\left(\mathrm{Sh}_{d} g\right)=\lambda_{\phi}(p) \mathrm{Sh}_{d} g$ for all $p>2$. Hence by the strong multiplicity one theorem it follows that $\mathrm{Sh}_{d} g$ is a constant multiple of $\phi$. Since $\phi$ is Hecke normalized we conclude that $\mathrm{Sh}_{d} g=b_{g, \infty}(d) \phi$, and that by necessity $b_{g, \infty}(d) \neq 0$. Therefore,

$$
k \sum_{\substack{m n=k \\ m, n>0}} \frac{1}{n^{3 / 2}}\left(\frac{d}{n}\right) b_{g, \infty}\left(d m^{2}\right)=b_{g, \infty}(d) \lambda_{\phi}(k) .
$$

Using Möbius inversion

$$
m b_{g, \infty}\left(d m^{2}\right)=b_{g, \infty}(d) \sum_{k \mid m} \frac{\mu(k)}{\sqrt{k}}\left(\frac{d}{k}\right) \cdot \lambda_{\phi}\left(\frac{m}{k}\right)
$$

and then applying the bound $\left|\lambda_{\phi}(k)\right| \ll k^{\theta+\varepsilon}$, where $\theta$ is the best exponent towards the Ramanujan-Petersson conjecture, gives $\left|b_{g, \infty}\left(d \delta^{2}\right)\right|^{2} \ll\left|b_{g, \infty}(d)\right|^{2} \delta^{2 \theta-2+\varepsilon}$.

\section{Appendix: The functional equation}

As promised we include for completeness the proof of Lemma 8.2. As already pointed out this follows very closely the proof given for holomorphic forms by Kohnen and Zagier [19].

Proof of Lemma 8.2. Let

$$
E_{\infty}^{(4)}(z, s)=\sum_{\gamma \in \Gamma_{\infty} \backslash \Gamma_{0}(4)}(\operatorname{Im}(\gamma z))^{s}
$$

be the Eisenstein series for $\Gamma_{0}(4)$ at the cusp at $\infty$ and

$$
E_{\infty}(z, s)=\sum_{\gamma \in \Gamma_{\infty} \backslash \mathrm{SL}_{2}(\mathbb{Z})}(\operatorname{Im}(\gamma z))^{s}
$$

the Eisenstein series for the full modular group. By the unfolding method,

$$
(4 \pi)^{-s+1} G(s)=\iint_{\Gamma_{0}(4) \backslash \mathbb{H}}|g(z)|^{2} E_{\infty}^{(4)}(z, s) \operatorname{dvol}(z) .
$$

Since $E_{\infty}^{(4)}(z, s)$ has a simple pole at $s=1$ with residue $\operatorname{vol}\left(\Gamma_{0}(4) \backslash \mathbb{H}\right)=(2 \pi)^{-1}$ we conclude that the residue of $G(s)$ at $s=1$ is

$$
\frac{1}{2 \pi} \iint_{\Gamma_{0}(4) \backslash \mathbb{H}}|g(z)|^{2} d \operatorname{vol}(z)=\frac{1}{2 \pi} .
$$

We would like to also conclude from the above representation that $G(s)$ has a functional equation relating $s$ to $1-s$, but this is not completely immediate since $E_{\infty}^{(4)}(z, 1-s)$ is a linear combination of Eisenstein series at the cusps of $\Gamma_{0}(4)$. In fact to establish the functional equation we will use the fact that $g \in V^{+}$. Recall 
that $V^{+}$corresponds to the subspace of $V$ with eigenvalue 1 of a certain operator $L$. Following Katok-Sarnak [17] we will now provide an explicit description of $L$. Consider the involution

$$
\tau_{2} F(z)=e^{i \pi / 4}\left(\frac{z}{|z|}\right)^{-1 / 2} F\left(\frac{-1}{4 z}\right)
$$

and the Hecke operator

$$
\sigma F(z)=\frac{\sqrt{2}}{4} \sum_{v} F\left(\frac{z+v}{4}\right)
$$

Then $L=\tau_{2} \sigma$. For a $g \in V^{+}$we have $L g=g$, and with the explicit description of $L$ this implies an identity that we will use. First notice that,

$$
\sigma g=\sqrt{2} g_{0}
$$

where for $j=0,1$,

$$
g_{j}(z)=\sum_{\substack{n \neq 0 \\ n \equiv j}} b_{g, \infty}(n) W_{\frac{1}{4} \operatorname{sgn}(n), i t}(\pi|n| y) e(n x / 4) .
$$

Therefore $L g=g$ is equivalent to $\sqrt{2} \tau_{2} g_{0}(z)=g(z)$. Since $\tau_{2}$ is an involution this means that $\sqrt{2} g_{0}(z)=\tau_{2} g(z)$. Hence from $\sqrt{2} \tau_{2} g_{0}(z)=g(z)$ and $\sqrt{2} g_{0}(z)=\tau_{2} g(z)$ we obtain the following two identities,

$$
\begin{gathered}
\sqrt{2} g_{0}(z)=e^{\pi i / 4}\left(\frac{z}{|z|}\right)^{-1 / 2} g\left(\frac{-1}{4 z}\right) \\
e^{\pi i / 4}\left(\frac{z}{|z|}\right)^{-1 / 2} g_{0}\left(\frac{-1}{4 z}\right)=\frac{1}{\sqrt{2}} g(z)
\end{gathered}
$$

In addition, using the Fourier expansion of $g$, and the above two properties we get,

$$
\begin{aligned}
e^{\pi i / 4}\left(\frac{z}{|z|}\right)^{-1 / 2} \cdot g\left(\frac{1}{2}-\frac{1}{4 z}\right) & =e^{\pi i / 4}\left(\frac{z}{|z|}\right)^{-1 / 2} \cdot\left(2 g_{0}\left(-\frac{1}{z}\right)-g\left(\frac{-1}{4 z}\right)\right) \\
& =\sqrt{2}\left(g\left(\frac{z}{4}\right)-g_{0}(z)\right)=\sqrt{2} g_{1}(z)
\end{aligned}
$$

using in both equations the fact that the coefficients $n \equiv 2,3(\bmod 4)$ of $g(z)$ are equal to zero. Now since $g_{0}$ and $g_{1}$ are automorphic forms of half-integral weight for $\Gamma_{0}(4)$ (since $\sigma: V \rightarrow V, \tau: V \rightarrow V$ and $V$ is a Hilbert space) by the Rankin-Selberg 
method we get

$$
\begin{aligned}
\pi^{-s+1} G(s)= & \iint_{\Gamma_{0}(4) \backslash \mathbb{H}}\left|g_{1}(z)\right|^{2} E_{\infty}^{(4)}(z, s) \operatorname{dvol}(z)+\iint_{\Gamma_{0}(4) \backslash \mathbb{H}}\left|g_{0}(z)\right|^{2} E_{\infty}^{(4)}(z, s) \operatorname{dvol}(z) \\
= & \frac{1}{2} \iint_{\Gamma_{0}(4) \backslash \mathbb{H}}\left|g\left(-\frac{1}{4 z}\right)\right|^{2} E_{\infty}^{(4)}(z, s) \operatorname{dvol}(z) \\
& \quad+\frac{1}{2} \iint_{\Gamma_{0}(4) \backslash \mathbb{H}}\left|g\left(\frac{1}{2}-\frac{1}{4 z}\right)\right|^{2} E_{\infty}^{(4)}(z, s) \operatorname{dvol}(z) \\
= & \frac{1}{2} \iint_{\Gamma_{0}(4) \backslash \mathbb{H}}|g(z)|^{2} \cdot\left(E_{\infty}^{(4)}\left(\frac{-1}{4 z}, s\right)+E_{\infty}^{(4)}\left(\frac{-1}{4 z+2}, s\right)\right) \operatorname{dvol}(z)
\end{aligned}
$$

Since (see for example Kohnen-Zagier, p. 191)

$$
E_{\infty}^{(4)}\left(-\frac{1}{4 z}, s\right)+E_{\infty}^{(4)}\left(-\frac{1}{4 z+2}, s\right)=\frac{1}{4^{s}-1}\left(2^{s} E_{\infty}(2 z, s)-E_{\infty}(4 z, s)\right)
$$

we conclude that

$$
\text { (11.2) } \pi^{-s+1} G(s)=\frac{1}{2} \iint_{\Gamma_{0}(4) \backslash \mathbb{H}}|g(z)|^{2} \cdot\left(\frac{1}{4^{s}-1}\left(2^{s} E_{\infty}(2 z, s)-E_{\infty}(4 z, s)\right)\right) \operatorname{dvol}(z)
$$

But by (11.1) and the identity

$$
E_{\infty}^{(4)}(z, s)=\frac{1}{4^{s}-1}\left(E_{\infty}(4 z, s)-2^{-s} E(2 z, s)\right)
$$

we also get

$$
\pi^{-s+1} G(s)=4^{s-1} \iint_{\Gamma_{0}(4) \backslash \mathbb{H}}|g(z)|^{2} \cdot\left(\frac{1}{4^{s}-1}\left(E_{\infty}(4 z, s)-2^{-s} E_{\infty}(2 z, s)\right)\right) \operatorname{dvol}(z) .
$$

Multiplying both sides of (11.3) by two, then adding (11.2) and dividing both sides of the resulting formula by three, we get

$$
\pi^{-s+1} G(s)=\frac{1}{6} \iint_{\Gamma_{0}(4) \backslash \mathbb{H}}|g(z)|^{2} E_{\infty}(4 z, s) \operatorname{dvol}(z) .
$$

Since $\pi^{-s} \Gamma(s) \zeta(2 s) E_{\infty}(4 z, s)$ is invariant under $s \mapsto 1-s$ we conclude that

$$
\widetilde{G}(s)=\pi^{-2 s} \Gamma(s) \zeta(2 s) G(s)
$$

is invariant under $s \mapsto 1-s$ as claimed. 


\section{Acknowledgments}

This paper is an outgrowth of our joint work with Kaisa Matomäki [22] and we are especially thankful to her for her numerous ideas and suggestions. We are also very grateful to Zeév Rudnick and Peter Sarnak for many conversations related to this project and for their encouragement. We would also like to thank Kannan Soundararajan for many discussions on moments and Valentin Blomer for very useful pointers to the literature on Whittaker functions.

\section{REFERENCES}

1. Ehud Moshe Baruch and Zhengyu Mao, A generalized Kohnen-Zagier formula for Maass forms, J. Lond. Math. Soc. (2) 82 (2010), no. 1, 1-16. MR 2669637 (2012c:11100)

2. A. Biró, Cycle integrals of Maass forms of weight 0 and Fourier coefficients of Maass forms of weight 1/2, Acta Arith. 94 (2000), no. 2, 103-152. MR 1779112 (2001g:11062)

3. Vorrapan Chandee, Explicit upper bounds for L-functions on the critical line, Proc. Amer. Math. Soc. 137 (2009), no. 12, 4049-4063. MR 2538566 (2010i:11134)

4. Y. Colin de Verdière, Ergodicité et fonctions propres du laplacien, Comm. Math. Phys. 102 (1985), no. 3, 497-502. MR 818831 (87d:58145)

5. W. Duke, Hyperbolic distribution problems and half-integral weight Maass forms, Invent. Math. 92 (1988), no. 1, 73-90. MR 931205 (89d:11033)

6. W. Duke, O. Imamoglu, and A. Tóth, Geometric invariants for real quadratic fields, pre-print availabe at www.math.ucla.edu/ wdduke/preprints/geometric.pdf (2015).

7. P. D. T. A. Elliott, C. J. Moreno, and F. Shahidi, On the absolute value of Ramanujan's $\tau$-function, Math. Ann. 266 (1984), no. 4, 507-511. MR 735531 (85f:11030)

8. Amit Ghosh, Andre Reznikov, and Peter Sarnak, Nodal domains of Maass forms I, Geom. Funct. Anal. 23 (2013), no. 5, 1515-1568. MR 3102912

9. Adam Harper, Sharp conditional bounds for moments of the riemann zeta function, Available on the arXiv arXiv:1305.4618 (2013), 20 pages.

10. D. R. Heath-Brown, Convexity bounds for L-functions, Acta Arith. 136 (2009), no. 4, 391-395. MR 2476604 (2010b:11115)

11. Roman Holowinsky, Sieving for mass equidistribution, Ann. of Math. (2) 172 (2010), no. 2, 1499-1516. MR 2680498 (2011i:11060)

12. Roman Holowinsky and Kannan Soundararajan, Mass equidistribution for Hecke eigenforms, Ann. of Math. (2) 172 (2010), no. 2, 1517-1528. MR 2680499 (2011i:11061)

13. H. Iwaniec and P. Sarnak, $L^{\infty}$ norms of eigenfunctions of arithmetic surfaces, Ann. of Math. (2) 141 (1995), no. 2, 301-320. MR 1324136 (96d:11060)

14. Henryk Iwaniec, Fourier coefficients of modular forms of half-integral weight, Invent. Math. 87 (1987), no. 2, 385-401. MR 870736 (88b:11024)

15. _ Topics in classical automorphic forms, Graduate Studies in Mathematics, vol. 17, American Mathematical Society, Providence, RI, 1997. MR 1474964 (98e:11051)

16. Dmitry Jakobson, Quantum unique ergodicity for Eisenstein series on $\mathrm{PSL}_{2}(\mathbf{Z}) \backslash \mathrm{PSL}_{2}(\mathbf{R})$, Ann. Inst. Fourier (Grenoble) 44 (1994), no. 5, 1477-1504. MR 1313792 (96b:11068)

17. Svetlana Katok and Peter Sarnak, Heegner points, cycles and Maass forms, Israel J. Math. 84 (1993), no. 1-2, 193-227. MR 1244668 (94h:11051) 
18. Henry H. Kim, Functoriality for the exterior square of $\mathrm{GL}_{4}$ and the symmetric fourth of $\mathrm{GL}_{2}$, J. Amer. Math. Soc. 16 (2003), no. 1, 139-183, With appendix 1 by Dinakar Ramakrishnan and appendix 2 by Kim and Peter Sarnak. MR 1937203 (2003k:11083)

19. W. Kohnen and D. Zagier, Values of L-series of modular forms at the center of the critical strip, Invent. Math. 64 (1981), no. 2, 175-198. MR 629468 (83b:10029)

20. Winfried Kohnen, Modular forms of half-integral weight on $\Gamma_{0}(4)$, Math. Ann. 248 (1980), 249-266.

21. Tomio Kubota, Elementary theory of Eisenstein series, Kodansha Ltd., Tokyo; Halsted Press [John Wiley \& Sons], New York-London-Sydney, 1973. MR 0429749 (55 \#2759)

22. Stephen Lester, Kaisa Matomäki, and Maksym Radziwiłl, Small scale distribution of zeros and mass of modular forms, Available on the arXiv arXiv:1501.01292 (2015), 31 pages.

23. Xiannan Li, Upper bounds on L-functions at the edge of the critical strip, Int. Math. Res. Not. IMRN (2010), no. 4, 727-755. MR 2595006 (2011a:11160)

24. Elon Lindenstrauss, Invariant measures and arithmetic quantum unique ergodicity, Ann. of Math. (2) 163 (2006), no. 1, 165-219. MR 2195133 (2007b:11072)

25. W. Luo, Z. Rudnick, and P. Sarnak, On Selberg's eigenvalue conjecture, Geom. Funct. Anal. 5 (1995), no. 2, 387-401. MR 1334872

26. Wen Zhi Luo and Peter Sarnak, Quantum ergodicity of eigenfunctions on $\operatorname{PSL}_{2}(\mathbf{Z}) \backslash \mathbf{H}^{2}$, Inst. Hautes Études Sci. Publ. Math. (1995), no. 81, 207-237. MR 1361757 (97f:11037)

27. Wenzhi Luo, Zeév Rudnick, and Peter Sarnak, The variance of arithmetic measures associated to closed geodesics on the modular surface, J. Mod. Dyn. 3 (2009), no. 2, 271-309. MR 2504745 (2010d:11055)

28. Simon Marshall, Mass equidistribution for automorphic forms of cohomological type on GL 2 , J. Amer. Math. Soc. 24 (2011), no. 4, 1051-1103. MR 2813338 (2012i:11049)

29. Roland Matthes, Rankin-Selberg method for real analytic cusp forms of arbitrary real weight, Math. Z. 211 (1992), no. 1, 155-172. MR 1179786 (93i:11059)

30. Fourier coefficients of real analytic cusp forms of arbitrary real weight, Acta Arith. 65 (1993), no. 1, 1-14. MR 1239239 (95d:11053)

31. Ritabrata Munshi, On quadratic families of CM elliptic curves, Trans. Amer. Math. Soc. 363 (2011), no. 8, 4337-4358.

32. Paul D. Nelson, Equidistribution of cusp forms in the level aspect, Duke Math. J. 160 (2011), no. 3, 467-501. MR 2852367

33. _ Mass equidistribution of Hilbert modular eigenforms, Ramanujan J. 27 (2012), no. 2, 235-284. MR 2886501

34. Paul D. Nelson, Ameya Pitale, and Abhishek Saha, Bounds for Rankin-Selberg integrals and quantum unique ergodicity for powerful levels, J. Amer. Math. Soc. 27 (2014), no. 1, 147-191. MR 3110797

35. S. Niwa, On Shimura's trace formula, Nagoya Math. J. 66 (1977), 183-202.

36. Yiannis N. Petridis, Nicole Raulf, and Morten S. Risager, Double Dirichlet series and quantum unique ergodicity of weight one-half Eisenstein series, Algebra Number Theory 8 (2014), no. 7, 1539-1595. MR 3272275

37. Maksym Radziwiłl and K. Soundararajan, Moments and distribution of central L-values of quadratic twists of elliptic curves, Invent. Math. 202 (2015), no. 3, 1029-1068. MR 3425386

38. Zeév Rudnick, On the asymptotic distribution of zeros of modular forms, Int. Math. Res. Not. (2005), no. 34, 2059-2074. MR 2181743 (2006k:11099) 
39. Zeév Rudnick and Peter Sarnak, The behaviour of eigenstates of arithmetic hyperbolic manifolds, Comm. Math. Phys. 161 (1994), no. 1, 195-213. MR 1266075 (95m:11052)

40. J.-P. Serre, A course in arithmetic, Springer-Verlag, New York-Heidelberg, 1973, Translated from the French, Graduate Texts in Mathematics, No. 7. MR 0344216 (49 \#8956)

41. Bernard Shiffman and Steve Zelditch, Distribution of zeros of random and quantum chaotic sections of positive line bundles, Comm. Math. Phys. 200 (1999), no. 3, 661-683. MR 1675133 (2001j:32018)

42. Goro Shimura, On modular forms of half integral weight, Ann. of Math. (2) 97 (1973), 440-481. MR 0332663 (48 \#10989)

43. T Shintani, On construction of holomorphic cusp forms of half-integral weight, Nagoya Math. J. 58 (1975), 83-126.

44. Lior Silberman and Akshay Venkatesh, On quantum unique ergodicity for locally symmetric spaces, Geom. Funct. Anal. 17 (2007), no. 3, 960-998. MR 2346281 (2009a:81072)

45. A. I. Šnirel'man, Ergodic properties of eigenfunctions, Uspehi Mat. Nauk 29 (1974), no. 6(180), 181-182. MR 0402834 (53 \#6648)

46. K. Soundararajan and Matthew P. Young, The second moment of quadratic twists of modular Lfunctions, J. Eur. Math. Soc. (JEMS) 12 (2010), no. 5, 1097-1116. MR 2677611 (2011g:11097)

47. Kannan Soundararajan, Moments of the Riemann zeta function, Ann. of Math. (2) 170 (2009), no. 2, 981-993. MR 2552116 (2010i:11132)

48. _ Quantum unique ergodicity for $\mathrm{SL}_{2}(\mathbb{Z}) \backslash \mathbb{H}$, Ann. of Math. (2) 172 (2010), no. 2, 15291538. MR 2680500 (2011j:11098)

49. _ Weak subconvexity for central values of L-functions, Ann. of Math. (2) 172 (2010), no. 2, 1469-1498. MR 2680497 (2011i:11077)

50. _ Weak subconvexity for central values of L-functions, Ann. of Math. (2) 172 (2010), no. 2, 1469-1498. MR 2680497

51. Raphael Steiner, Supnorm of modular forms of half-integral weight in the weight aspect, Available on the arXiv arXiv:1504.08246 (2015), 13 pages.

52. J.-L. Waldspurger, Sur les coefficients de Fourier des formes modulaires de poids demi-entier, J. Math. Pures Appl. (9) 60 (1981), no. 4, 375-484. MR 646366 (83h:10061)

53. Thomas C. Watson, Rankin triple products and quantum chaos, Available on the arXiv arXiv:0810.0425 (2008), 58 pages.

54. Steven Zelditch, Uniform distribution of eigenfunctions on compact hyperbolic surfaces, Duke Math. J. 55 (1987), no. 4, 919-941. MR 916129 (89d:58129)

Department of Mathematics, KTH Royal Institute of Technology, SE-100 44 STOCKHOLM, SWEDEN

E-mail address: sjlester@gmail.com

Department of Mathematics, McGill University, 845 Rue Sherbrooke Ouest, Montréal, QuÉBEC H3A 0G4, CANADA

E-mail address: maksym.radziwill@gmail.com 